\title{
Concordant developmental expression profiles of orthologues in highly divergent Bilateria
}

\author{
Luca Ferrettia ${ }^{\mathrm{a}, \mathrm{b}, 1, *}$, Andrea Krämer-Eis ${ }^{\mathrm{a}}$, Philipp H. Schifferª,c,1 \\ ${ }^{a}$ Institut für Genetik, Universität zu Köln, Zülpicher Straße 47a, 50674 Köln, Germany \\ ${ }^{b}$ Big Data Institute, Li Ka Shing Centre for Health Information and Discovery, Nuffield \\ Department of Medicine, University of Oxford, UK \\ ${ }^{c}$ Center for Life's Origin and Evolution, GEE, University College London, London
} WC1E 6BT, UK

\begin{abstract}
Bilateria are the predominant clade of animals on earth. Despite having evolved a large variety of body-plans and developmental modes, they are characterized by common morphological traits. However, it is not clear if clade-specific genes can be linked to these traits, distinguishing bilaterians from non-bilaterians, with their less complex body morphology. Comparing proteomes of bilaterian and non-bilaterian species in an elaborate computational pipeline we aimed to find and define a set of of bilaterian-specific genes. Finding no high-confidence set of such genes, we nevertheless detected an evolutionary signal possibly uniting the highly diverse bilaterian taxa. Using a novel multi-species GO-enrichment method, we determined the functional repertoire of genes that are widely conserved among Bilateria. We found that these genes contribute to morphogenesis, neuronal-system and muscle development, processes that have been described as different between bilaterians and non-bilaterians. Analyzing gene expression profiles in three very distantly related bilaterina species, we find characteristic peaks at comparable stages of development and a delayed onset of expression in embryos. In particular, the expression of the conserved genes appears to peak at the phylotypic stage of different bilaterian phyla. In summary, our data underpin

\footnotetext{
* corresponding authors

Email addresses: luca.ferretti@bdi.ox.ac.uk (Luca Ferretti), a.kraemer .eis@googlemail.com (Andrea Krämer-Eis), p.schiffer@ucl.ac.uk (Philipp H. Schiffer*)

${ }^{1}$ Equally contributing
} 
the orthologue conjecture and illustrate how development connects distantly related Bilateria after millions of years of divergence, pointing to processes potentially separating them from non-bilaterians.

Keywords: Evolution, Bilateria, Gene Ontology, Expression profile, Development

\section{Introduction}

Bilateria represent by far the largest monophyletic group in the animal kingdom. They comprise about $99 \%$ of the extant eumetazoans [1] and are classified into 32 phyla [2]. The taxon 'Bilateria' has been defined on the basis of morphological key innovations, namely bilateral symmetry, triploblasty, an enhanced nervous system and a complex set of cell types [3]. Probably the most striking observation is the existence of a large variety of body plans, accompanied by high morphological diversity in many bilaterian phyla [4].

It is thought that the major bilaterian phyla emerged in a fast radiation during the early Cambrian, about 540 million years ago [1], but the appearance of the common bilaterian ancestor, and its exact timing, is controversial $[5,6,7,8]$. It is also difficult to define unifying morphological properties for larval or adult Bilateria, as their descendants underwent extensive re-modellings (including secondary reductions and simplifications) of body forms in the lineages leading to extant crown clades. Nevertheless, the general process of development from single-cell to adult is conserved among all animals. Recently, evidence for the hypothesis that the developmental transcriptome might be a conserved trait across diverse groups of animals has been found $[9,10]$. In particular, several studies described a conserved phylotypic period mid development based on transcriptomic analyses $[11,12,13]$, akin to the morphological hourglass model of developmental progression [14, 15], itself an extension of von Baers reverse funnel model of development in animals [16].

Despite these similarities in the global transcriptome, comparisons at the molecular level reveal that many developmentally important genes are older than the ancestor of Bilateria. Among them are determinants of dorso-ventral and anterior-posterior patterning, eye formation, segmentation, and heart development $[5,17,18,19,20]$. In a recent study, it was estimated that up to $85 \%$ of the genes present in any Bilaterian examined were already present in the ur-bilaterian [21], and only about $15 \%$ are of more recent 
origin. Deep comparative genomics including non-bilaterian Metazoans, also revealed that the major developmental signaling pathways are already present in Cnidarians, Ctenophores, Placozoa and sponges [22, 23, 24], and must predate the origin of Bilateria. At the same time, it was shown that genes specific to large clades with divergent sub-taxa, like the deuterostomes, might exist [25]. We hypothesised that similar to the conserved developmental transcriptome and the gene novelties described to define the deuterostomes [25] there might be genes defining the Bilateria as a clade, setting them apart from their non-bilaterian ancestors.

Using reciprocal blast searches, orthology pipelines, and an array of filtering steps we identified a set of orthologues proteins that satisfy our initial criteria for Bilateria specificity. However, these proteins could not be determined to be actually clade-specific for Bilateria with highest-confidence after secondary validation. In fact, re-analysing an initial set of 85 orthogroups with new data from the ever growing database of available proteins we found only 35 orthogroups to remain "Bilateria-specific". It must be assumed that this set would shrink even further with more data becoming available, in particular from more non-bilaterians.

Despite our limited confidence in the ability of these approaches to detect clade-specific genes in Bilateria, we observed an interesting signal in our data. Analysing the set of 85 orthogroups in more detail we found that these genes are not only retained in species separated by a billion years of independent lineage evolution, but that most of them act in key developmental processes. These genes show very similar and peculiar expression profiles across analogous developmental stages in highly derived model species in the fishes, the arthropods, and the nematodes. This finding supports the idea that evolutionary changes in the genetic machinery of development were coupled to the emergence of Bilateria and are still conserved in very divergent taxa today. Additionally, by finding similar developmental signals across the expression profiles for the most conserved orthologues in our orthogroups, our work also lends new support to the ortholog conjecture.

\section{Results}

\subsection{Defining potential clade-specific proteins in Bilateria}

The aim of our study was to identify genes that newly emerged in the 'ur-bilaterian' (Fig 1a) and have been retained in its descendant species. 
To this end, our initial study was grounded in a comparison of ten bilaterian and seven non-bilaterian species with fully sequenced and annotated genomes as of 2011. We downloaded 383,586 protein sequences in total. About $70 \%(268,252)$ are from Bilateria, covering the three major clades Lophotrochozoa, Ecdysozoa and Deuterostomia, and about 30\% (115,334) are non-bilaterian (Table 1). We discarded all bilaterian sequences that had a best BLAST hit below the threshold $E$-value of $10^{-5}$ with non-bilaterian sequences. We retained 13, 582 bilaterian specific candidate proteins which were grouped into 1,867 clusters of orthologous proteins (COPs) using the OrthoMCL pipeline [26]. We condensed the raw set of 1,867 clusters of orthologous proteins by applying filters with different stringency for the taxa included (Fig 1) and obtained the following four sets

$C$ : Each cluster must contain one or more representatives from each of the three major clades, Lophotrochozoa, Ecdysozoa and Deuterostomia. This set contains 506 clusters.

$M$ : Each cluster must contain representatives from all model organisms, $D$. rerio, D. melanogaster and C. elegans. This set contains 160 clusters.

$L$ : Each cluster contains representatives from all major clades, as in set $C$. However, only those clusters are permitted for which the representation of species is explained by at most one loss event along the species tree (set $C$ does not have this restriction). This resulted in 125 clusters (see supplementary Table S1)

A: Each cluster contains representatives of all bilaterian species considered (no protein loss is admitted) except possibly A. californica. This set has 34 clusters.

On average, each of the nine bilaterian species is represented in $66.2 \%$ of the 506 ortholog clusters in set $C$. In set $M$, this percentage is $80.9 \%$, and in set $L$ it is $91.2 \%$ (see Table 2), reflecting the levels of stringency of the filtering criteria.

\subsection{Orthologues conserved across divergent Bilateria}

In our analysis, we included the highly divergent bilaterian model organisms Caenorhabditis elegans, Drosophila melanogaster, and Danio rerio. 
These have very well curated and annotated genomes and are therefore helpful to find genes which are also functionally conserved since more than $500 \mathrm{Myrs}$ of independent evolution. To take advantage of this, we decided to use the intersection of sets $M$ and $L$ (termed $L^{\prime}=L \cap M$ ) for further analysis. Compared to set $L$ (125 clusters), this intersection lacks 31 clusters missing a $C$. elegans ortholog and 9 clusters missing an ortholog from $D$. melanogaster, resulting in a set of 85 orthogroups. Except for A. californica, the least represented species in these clusters is $S$. purpuratus (genes of this species occur in 63 of 85 clusters). By construction the model organisms $(C$. elegans, D. melanogaster, D. rerio) are represented in all clusters in $L^{\prime}$. On average, a (bilaterian) species is represented in $93.1 \%$ of the clusters.

\subsection{Clade-specificity declines with data availability}

A general drawback of our experimental procedure is its reliance on correctly identified and annotated genes. Since the majority of the organisms in our study are non-model organisms, they may suffer from incomplete or erroneous gene annotation. For example, we omitted the bilaterian species A. californica from downstream analyses because of its low quality protein annotation. It is also possible that truly existing genes are missing from the non-bilaterian dataset due to annotation or prediction errors or simple insufficient availability of sequenced species, leading to wrong inferences of bilaterian specificity. To test this possibility, we first verified by additional BLAST searches at NCBI that none of the 85 COPs in set $L^{\prime}$ had BLAST hits below an $E$-value of $10^{-5}$ in non-bilaterians or other eukaryotes available in August 2014. To accomodate to the very fast growing amount of data from across the bilateria and non-bilateria becoming available in recent years, we decided to implement a second, more comprehensive test to validate the potential bilaterian specificity set $L^{\prime}$. Conducting searches with Diamond and NCBI-BLAST against a wide array of protein data downloaded in August 2017 from NCBI we identified potential homologs for proteins in the 85 COPs. Using OMA and phylogenetic inferences we could then identify nonbilaterian orthologs for all but 35 of the 85 set $L^{\prime}$ COPs. This showed that with additional genomes and transcriptomes becoming available, the number of bilaterian specific orthologous groups decreased drastically.

\subsubsection{Most conserved orthologues}

Althoug not being strictly bilaterian-specific, we regarded the set $L^{\prime}$ COPs as biologically interesting since they are conserved across huge evolutionary 
distances with many speciation events separating them in the three model organisms (and additionally divergent enough from non-bilaterian sequences to not be detected in our first re-BLAST screen). We thus wondered what their biological function is and if this function could be correlated to a distinct process in the biology of Bilateria. As a result of our experimental design, but also due to different duplication histories, all COPs contain differing numbers of paralogs from different species. On average, we found most paralogs in Deuterostomia, followed by Ecdysozoa and Lophotrochozoa. The highest average paralog number is found for $D$. rerio, which might be a consequence of the additional genome duplication events in Teleosts [27]. Since the function of paralogs might differ from the function in the last common ancestor, we constructed an additional set of ortholog clusters free of paralogs: we extracted from each cluster in our original set $L^{\prime}$ the most conserved ortholog for each species, according to the PhastCons conservation score [28], and discarded the less conserved paralogs. This reduction does not affect the number of clusters, but the number of proteins within a cluster. In our downstream analyses we considered both versions, the 'most conserved orthologs' (MCO) and the 'all orthologs' (AO) catalog for each cluster.

\subsection{Gene ontology and functional classification}

GO terms reveal a link to development

To gain an overview over the biological functions of the proteins in set $L^{\prime}$, we extracted and analyzed their associated GO terms. To quantify simultaneous enrichment in multiple species, we developed a novel generalized Fisher's exact test for multiple species, which we term MSGEA ('Multi Species GO Enrichment Analysis'; Fig 3 and Fig S1). In contrast to standard GO enrichment analysis, MSGEA does not solely focus on the over-representation of GO terms in single species, but is able to detect GO terms enriched across several species, even if not over-represented in any single species. Of special interest for our analysis were GO terms occurring across all three model organisms: such terms may indicate the conservation of biological function across large evolutionary time scales. To our knowledge, this novel method is the only test that is sensitive to coherent enrichment of GO terms across multiple species (see Methods). Applying MSGEA we extracted a list of GO terms which are likely associated to long-term retained functions of our candidate genes. Concentrating on the domain 'biological process' of the GO database, we find the terms 'development', 'muscle', 'neuron', 'signaling' and 
'regulation' to be strongly over-represented (Fig. 4 and supplementary Table S4). The terms identified through in silico analysis suggest a prominent role for these orthologues in development.

\section{Inference of biological function through literature mining}

Since orthology is a strictly phylogenetic criterion, and since GO classification draws heavily on sequence homology, without necessarily reflecting conserved function, we decided to perform an in-depth human-curated literature search to collect additional evidence for the functional role of genes in COP clusters. We mined the literature databases and compiled human curated functional descriptions, based on experimental evidence, for the proteins contained in set $L^{\prime}$. We defined the six classes 'Neuron related', 'Morphology related', 'Muscle related', 'Signaling related', 'Regulation related' and 'Others' (supplementary Table S5). We used functional descriptions extracted from the literature to assign each of the 85 set $L^{\prime}$ clusters to one of these six classes (Fig. 4). The manual extraction of species-and proteinspecific functional information allowed us to compare and better interpret functions across the three model organisms.

To compare this human-curated classification with the one based on the GO database, we assigned all GO terms appearing in set $L^{\prime}$ to one of the six classes above. Since a given protein may be associated with many GO terms there is no one-to-one relationship of the human curated annotation and the GO terms. For example, while the human curation assigns eight of the 85 clusters (the left-most columns in Fig 4) to the category 'Morphology related', morphology related GO terms occur in 33 clusters, scattered throughout the six classes. Similarly, signaling related GO terms are found in almost every cluster, while the class 'Signaling related' contains only 18 clusters. However, our manual curation is still broadly consistent with GO annotations. For example, GO terms associated to 'muscle' do occur in the class 'Muscle related' and GO terms related to 'neuron' are mostly found in the 'Neuron related' clusters.

\subsection{Expression profiles}

To analyze expression of genes in set $L^{\prime}$ across developmental stages and to compare it between model organisms, we used publicly available data for D. melanogaster, D. rerio and C. elegans. We normalized expression values separately for each species and for each developmental stage - by the genomewide average, and then log-transformed them. 
Pooled profiles

To obtain a global picture of the expression profiles of genes in set $L^{\prime}$, we calculated mean and median profiles for each of the three model organisms, shown as orange lines in Fig 5 and Fig S3, top row. To see how expression of bilaterian-specific genes compares to background expression, we generated two different background sets for each species. The first is a randombackground, obtained by randomly selecting genes from the model organism genomes and recording their expression (distribution shown as white boxplots in Fig 5). The second background, which we call the 'matched-function' background (orange boxplots), is a random collection of genes with GO terms matched to those found in set $L^{\prime}$. The background distributions were constructed independently for each stage and for each set, and they may differ in size and content. We grouped the developmental stages from fertilized egg to the adult body into coarse grained, yet comparable, phases: 'Blastoderm', 'Gastrulation and Organogenesis', 'Hatching to Larva' and 'Adult' (separated by vertical lines in Fig 5).

For all three species, the set $L^{\prime}$ profiles roughly follow the shape of the background profiles across embryogenesis, however at a higher expression level (Fig 5). In D. melanogaster, expression of bilaterian-specific proteins is initially low, then rises until 'dorsal closure', after which it slighly decreases again. In $C$. elegans, expression is also initially low and then rises towards 'ventral enclosure'. This pattern is mirrored in D. rerio, where expression peaks around the pharyngula stage. The profiles seen in $C$. elegans and $D$. rerio are congruent with the major cycle of tissue proliferation and differentiation during organogenesis in these species [29]. During 'dorsal closure' in Drosophila, 'ventral closure' in C. elegans and 'pharyngula' in Danio, the head, the nervous system and the bilaterally organized body develop [30]. In Drosophila we find a second expression peak during a second cycle of proliferation corresponding to metamorphosis. The matched-function profile largely resembles the profile of set $L^{\prime}$. However, set $L^{\prime}$ genes show distinctly low expression in the very early embryonic stages, a pattern which is consistently seen in all species (Fig 5 and Fig S3). Another common feature is that expression of MCO genes is consistently higher than of AO genes (Fig 5).

\section{Characteristic expression profiles}

To further analyze the expression profiles, we clustered individual profiles based on their similarity and extracted a few typical ones for each species (Fig 5, bottom row). Methods exist to identify tissue-specific expression 
profiles, or to differentiate between profiles, but no standard techniques are available to detect enrichment of profiles across a set of genes. To overcome this limitation, we employed an ad hoc 'profile enrichment' strategy. Briefly, we considered a COP expression profile relevant, if similar profiles were significantly more abundant among genes in our COPs than among other genes (for details, see Methods). We extracted all statistically significant profiles and then clustered them to distill representative ones. For each of the three model species qualitatively similar patterns are retrieved: an intersection of increasing expression profiles (yellow and green lines in Fig 5, $2^{\text {nd }}$ row) with slightly decreasing profiles (dark red and dark blue lines). The intersection occurs right before the developmentally important events 'dorsal closure', 'ventral enclosure' and 'pharyngula'. A second crossing of profiles can be seen in the fly, just before metamorphosis into the adult body. To interpret this observation, we considered the following functional classification.

\section{Expression profiles stratified by function and age}

We determined the mean expression profiles for the six functional classes - morphology- (8 set $L^{\prime}$ COPs), muscle- (11), neuron- (23), signaling- (18), regulation-related (20) and other genes (5) (Fig S2). We find upward and downward peaked expression patterns for the classes morphogenesis, neuron and muscle development (Fig S4), which are even emphasized compared to the pooled profiles of the global analysis. This is in particular true for expression before the onset of gastrulation. On the contrary, the genes related to regulation and signaling display a rather constant profile, without the characteristic expression peaks of morphogenesis.

For species as different as animals, plants and fungi, it has been repeatedly observed that timing and level of expression during development depend on the evolutionary age of a protein [31, 32, 33, 30, 34]. For instance, one recurrent finding was that older genes tend to show higher expression during early embryogenesis. To examine our proteins in this respect, we analysed whether the set $L^{\prime}$ expression profiles are more similar to the profiles of younger or of older genes, and we calculated background distributions considering the age of genes. Genes which arose before Bilateria (phylostratigraphic level less than 7, corresponding to Bilateria, according to the strata-numbers by Domazet-Lošo) were defined as 'older' and all others (level > 7) as 'younger' [32]. Expression profiles of genes $(\mathrm{AO})$ in set $L^{\prime}$ match very well the profiles of the 'younger' genes with matched function (red lines and orange boxplots in Fig 5 and Fig S3). All three species show low expression of young and of 
set $L^{\prime}$ genes during early development. Hence, this late onset of expression appear to be an evolutionary novelty of Bilateria. After 'dorsal closure' in $D$. melanogaster, 'ventral closure' in C. elegans, and 'pharyngula' in D. rerio, expression increases and older and younger profiles converge.

\section{Discussion}

The most significant innovations of bilaterian animals are a third germ layer (the mesoderm), a liquid filled body cavity surrounded by mesoderm (the coelom), a complex nervous system, and a large number of specialised cell types. The formation and presence of these features had a massive effect on the evolutionary possibilities and success of bilaterians. By analysing the genomes of bilaterian and non-bilaterian species we aimed at testing whether there is a genomic correlate to these morphological key innovations. Roughly a billion years of divergence between the most distant species, chromosome fusions, fissions and re-arrangements, genome duplications, expansions and losses of gene families, as well as myriads of nucleotide substitutions might have blurred the common genetic heritage of Bilateria. However, those genes and proteins which have evolved under steady purifying selection, i.e. play a key non-redundant role in extant Bilateria species, should still be identifiable as orthologs across Bilateria. We searched for genes that potentially evolved in the bilaterian ancestor and have been retained in extant bilaterians because such genes might have influenced the evolution of bilaterians. We were initially able to detect a set of 85 orthogroups (set $L^{\prime}$ ), containing genes which are shared between widely divergent Bilateria and, at the same time, appeared to be without orthologues in non-Bilateria. We reason that this set had to be taken with caution, as for example there is no clear-cut threshold of sequence similarity which separates orthology from non-orthology. In such cases the decision, whether a gene with partial homology should be included into an orthogroup or not, is somewhat arbitrary. For the 85 set $L^{\prime}$ COPs we have considered partial homology to a non-bilaterian sequence as an insufficient criterion to exclude an orthogroup from our initial list. A similar BLAST strategy as implemented by us, however without subsequent orthology clustering, had been used before to determine the evolutionary origin of genes and to assign phylostratigraphic ages [35, 32, 36, 34]. One pitfall of this approach is that an overall alignment score, such as an Blast $E$-value, may lead to both false positive and false negative predictions. False positives arise from the (wrong) inclusion of a protein into the bilaterian-specific list, which 
does have non-bilaterian orthologs, but which are missed due to low sequence similarity or due to absence from the database. False negative predictions arise from missing truly bilaterian-specific proteins, for instance when a conserved domain is identified in a protein for which a homologous domain is also present in non-bilaterians. One such example is the $\mathrm{C} 2 \mathrm{H} 2$ zinc finger protein CTCF which has been described as bilaterian-specific before [37]. Our search did not recover this protein, because multi-zinc finger proteins exist in cnidarians and their conserved Cys/His residues and linker regions evoke a similarity above threshold. As a result, our approach will wrongly treat such proteins as older and not bilaterian-specific and, consequently, eliminate them. Similar errors may affect other proteins with extended conserved domains. However, proteins that pass the filter should have a high probability to be true bilaterian novelties. In contrast, genes may be wrongly assigned to subclades which are younger than bilateria. For instance, this could be a consequence of non-orthologous gene displacement (NOGD) [38], a process in which functions are taken over by new or different proteins and the original orthologue is lost. Finally, we could have wrongly classified a protein as bilaterian-specific because faint homology to more anciently diverged species is missed.

Another limitation in our initial screen was the restriction to 16 highquality genomes, from which we infered bilaterian specificity. Firstly, it has now been shown that OrthoMLC, which we mainly relied on, is limited in finding distantly related orthologues if taxon sampling is coarse [39]. This problem could have been inflated by our restriction of candidates in our blast searches before running orthology prediction pipelines. Secondly, new entries to the databases may change the inferred phylostratigraphic age of an orthogroup. Being aware of this and in particular because sequencing efforts are increasingly directed towards non-standard model organims in the last years, we have scrutinized and re-inspected our initial candidate set of set $L^{\prime}$ COPs with refined tools and new data. While the remaining sub-set of 35 orthogroups might consist of bilaterian-specific proteins, it is likely that more data from so far not-yet-sequenced non-bilaterian species will also reveal orthologous for some of these proteins. These caveats raise doubts on the likelihood to detect high-confidence genes that are unequivocally bilaterian and at the same time widely retained in this taxon. Our results clearly raise the possibility that there are just a handful of Bilateria-specific genes, or even none at all. At the same time our approach of partially falsifying the results of the first orthology screen by implementing two additional checks 
shows that if there is any such gene, careful and elaborate measures have to be taken to validate potential candidates. At the very least all available genomic and proteomic data from currently available databases have to be screened.

Despite not being necessarily clade-specific, our set of genes is retained in very distantly related bilaterian species, while at the same time not being restricted to the group of classical, universally-conserved house keeping genes. This is interesting to note since even highly conserved and important genes can become lost, such as some Hox genes, e.g. from C. elegans [40] and the nematodes in general. Our set is also significantly divergent from potential non-bilaterian orthologs, suggesting either their origin or their rapid evolution in the ur-bilaterian. It thus appeared logically to ask which functions these orthologues might have in bilaterian animals. In comparison to classical enrichment analysis implemented in various software packages like blast2GO [41], our MSGEA test allows for the first time to compare GO-enrichment across several species. This is advantageous in the age of genomics where very often more than one species or a number of samples is analysed. In our framework of three model bilaterians, a fruit fly, a zebrafish, and a roundworm, the MSGEA based GO term analysis and subsequent literature mining indicated roles in development for the clustered genes. This link appears likely, since the process of development, the construction of adult specimens from single cells, is conserved in all animals and is also the phase when the combined hallmarks of bilaterian life are brought together: bilateral symmetry, triploblasty, the enhanced nervous system, and a set of complex cell types. In particular, the 'muscle' and 'neuron' class of clusters is compatible with the idea that key bilaterian innovations involved the central nervous system and muscles [42]. In general the connection to development is in agreement with other studies, such as the large scale modEncode study of model organisms [29].

The conserved genes in set $L^{\prime}$ follow characteristic expression patterns during development, which are shared between species separated by a billion years of independent evolution. In particular, we observe characteristic low expression in early development and a peak towards the end of larval development common to highly derived taxa such as nematodes, insects and teleosts. The single (fish and nematode) and double peak (fly) patterns which we observed are in agreement with results from the modEncode project $[43,29]$. Starting from co-expression analyses, the authors arrived at a set of orthologous genes acting in comparable stages of D. melanogaster 
and $C$. elegans development. The simpler, single peaked pattern in C. elegans and $D$. rerio reflects up-regulation at ventral enclosure and pharyngula stages, respectively. Thus, the expression profiles in these organisms mirror their life-cycle, displaying shared peaks when the adult body-plan is assembled. This phase, dominated by morphogenesis, has been interpreted as the phylotypic stage in both organisms $[44,45]$. In the holometabolous fly the situation is more complex: a body morphology is built twice, for the larva and then for the adult. These are two phases of increased cell proliferation during gastrulation and the transition from pupa to adult. The two peaks are also visible in the matched-function set, but they are less pronounced in the random-background set. It thus appears the two peaks we observed for our proteins reflect up-regulation during both phases of cell proliferation. It will be interesting to analyse if similar patterns can be observed in other species undergoing metamorphosis, particularly in vertebrates.

It has been hypothesised that differences in gene regulatory processes and the deployment of GRNs may have been more important for the evolution of Bilateria than the conservation of single key genes [46]. A conserved profile of developmental gene expression was observed in several studies across animal phyla, including in non-bilaterians, when developmental time course data were analysed [12]. These studies suggest the existence of a phylotypic stage late in development, but see $[47,12,31]$. We observed low expression of young genes that originated after the emergence of Bilateria and of set $L^{\prime}$ genes in early development. We interpret this to indicate that early embryonic processes, e.g. initial cell divisions, are governed by evolutionary old genes, which are common to all bilateria and non-bilateria. Thus while, non-Bilateria have a similar complex distribution and abundance of gene regulatory elements and systems [48, 49], and likely their own phylotypic stage, our data support the idea that a change in gene regulation in the phase when adult morphology is shaped might be linked to the emergence of Bilateria. What is more, the process appears to be conserved across immense evolutionary distances.

In agreement with the general idea of the orthology-conjecture, paralogs contained in set AO may be sub-functionalized, while the MCO genes are more likely to retain their original function. This observation on the MCO genes appears interesting in regard to the ortholog conjecture, which posits that orthologues are more likely to retain function in divergent organisms in comparison to paralogues, see e.g. [50]. While being challenged in the past [51] more recent analyses have found support or at least some support 
for the ortholog conjecture by comparing for example mammals or divergent model organisms $[52,53,54]$. Our data does give additional support to the idea of functional conservation of orthologues in very distant species and in particular potentially extends the conjecture to expression patterns. That paralogues are indeed more divergent in their function was for example observed in flies [55] and appear apparent in our data of the AO subset as well.

\subsection{Conclusions}

In this study we performed a systematic approach to identify bilaterianspecific genes. Previous investigations concluded that essentially all important developmental regulators precede the origin of bilaterians, and that rewiring of already existing factors was a hallmark of bilaterian evolution $[24,56,23,22,48]$ and our results enhanced by a second BLAST and phylogeny based control step are in support of this. Future data from nonbilaterian species might in fact show that even the 35 groups of orthologues we now identified to be conserved across the three model species but without credible non-bilaterian orthologues were present before the split from Cnidaria. Conversely, it remains possible that genes independently lost in bilaterian crown groups have no non-bilaterian orthologues. Analysing the roles of genes we found conserved across crown groups in bilateria we found a strong link to development. Thus, our results indicate that Bilateria are unified by processes occuring late in embryonic development, which are shaped by a set of conserved genes. At the same time early developmental processes might be shared across Metazoa. Finally, our findings give new support to the ortholog conjuncture.

\section{Materials and Methods}

Using a comparative genomic approach, we aimed to identify genes shared among and specific to Bilateria. To represent the spectrum of the different bilaterian clades we selected ten species from the three major clades - Deuterostomia, Ecdysozoa and Lophotrochozoa - concentrating on species with a fully sequenced and annotated genome (Table 1). We included more species from Lophotrochozoa than from the other two clades, since well-annotated complete genome sequences from lophotrochozoan model-organisms were still under-represented in public databases at the beginning of our studies. To obtain a high contrast between Bilateria and Non-bilateria, we used seven 
non-bilaterian species, as in a previous study [37]. Proteomes and data from InterProScan [57] and the Gene Ontology consortium [58] were retrieved from the sources cited in Table 1.

\section{Initial BLAST and Clustering}

To find orthologs we set up a stringent analysis pipeline. We first species blasted all-versus-all among the 17 proteomes using the stand-alone BLAST version $2.2 .25+$ with a cutoff of $E=10^{-5}$. We then discarded all bilaterian proteins which had a significant hit in any of the non-bilaterian sequences. This filtering resulted in 13,582 candidate proteins. From these we constructed orthologous groups using two different ortholog finders, InParanoid and OrthoMCL [26, 59], using default parameter settings. Both programs were rated highly in benchmarking studies which analyzed the performance of orthology-prediction methods [60, 61]. For the purpose of detecting orthologs of very diverged species, we chose OrthoMCL version 2.0.2 and the associated MCL version 11-335, as they were shown to be more robust [60, 61] than similar programs. Finally, we grouped the ortholog clusters into four sets $(A$, $L, M, C$; Fig 1), satisfying different stringency conditions, see page 4 . Due to the poor sequence quality, we treated the Lophotrochozoon $A$. californica differently: where available we included $A$. californica orthologs, but we did not require them to be present in set $A$ ('all species').

The above data sets are ordered by degree of initial confidence in the bilaterian specificity of the respective proteins. At the same time they reflect the level of conservation of the proteins across different species. Seeking to be conservative regarding bilaterian specificity on the one hand (set $A$ being most conservative), but on the other hand to analyze a dataset which is as comprehensive as possible, we focused our further analyses on the intersection of sets $L$ and $M$ (called $L^{\prime}$; Fig 1) comprising 85 clusters.

\section{Validation with BLAST, OMA, and Phylogeny}

As our original dataset was bilaterian and non-bilaterian species was assembled prior to 2012 and the steady flow of newly sequenced species has added massive new numbers of protein predictions to online databases we wanted to re-evaluate the potential bilaterian-specificity of our 85 final clusters. To this end we downloaded 19,185,382 bilaterian and 497,273 nonbilaterian proteins from the NCBI database in August 2017 and created individual databases for taxa in bilateria and non-bilateria. Next we used Diamond [62] to blast the D. rerio, C. elegans, and D. melanogaster proteins 
for each of the 85 clusters against the individual taxa. Additionally, we used NCBI BLAST with the parameters described to be most sensitive in finding orthologs in [63] for the non-bilaterian taxa. For each of the 85 clusters we then collected the 10 best blastp hits per taxon. Along with the original model organism proteins we obtained 96,287 proteins in this way, which we used as input for the OMA orthology prediction pipeline [64]. From the OMA output we selected hierachical cluster of orthologues (HOG) for those of the original 85 clusters where the HOG contained non-bilaterian proteins. Finally, we aligned all sequences per HOG with clustal-omega [65] and reconstructed a phylogeny using IQTrees with automated model selection and 1000 fast bootstrap replicates [66]. We then analysed these trees by eye.

\section{Selecting most conserved orthologs}

For the three model species $D$. rerio, D. melanogaster, and $C$. elegans we extracted the UCSC tracks of basewise PhastCons conservation scores [28] calculated across insects, teleosts and nematodes, respectively. We used these scores to rank all genes in a cluster according to their fraction of strongly conserved sites, i.e. sites with PhastCons score $>0.99$. We selected the highest-ranking one from each cluster as the 'Most Conserved Ortholog' (MCO). These genes likely retained the same function, even in highly diverged species - a conjecture based on the idea that strong conservation reflects long-term evolutionary (and functional) constraint and that neo- or sub-functionalized paralogs tend to be less conserved. We used the fraction of strongly conserved sites, instead of the average conservation score, since we are interested in the degree of conservation in function, not in sequence. We reasoned that functional conservation should be related to high conservation of alleles at functional sites, while the remaining bases can evolve fast. However, the two ways of measuring similarity are highly correlated on a genome-wide scale across all coding exons $(r=0.91$ for D. melanogaster, 0.84 for D. rerio and 0.71 for C. elegans (Fig 2)). We performed all subsequent analyses on both sets: the set of 'All orthologs and paralogs' (AOs) and the set of MCOs.

\section{Multi-Species Gene Enrichment Analysis}

To examine function of the genes in set $L^{\prime}$ by in silico methods, we obtained their gene ontology (GO) terms for the three model species from the GO database [58]. To identify terms enriched in COP genes compared to the 
genomic background, we employed two strategies: the conventional singlespecies enrichment analysis based on Fisher's exact test and a novel method, termed multi-species gene enrichment analysis (MSGEA). Standard singlespecies Fisher's exact tests for enrichment cannot capture a signal of moderate, but joint, enrichment across different species. Instead, with MSGEA we compute an exact $p$-value for a pooled set of species, employing the following steps. First, for each GO term we counted the number of descendants in the GO tree. Then, we computed the $p$-values for the terms occurring in any species and corrected for multiple testing [67]. More specifically, let $n_{g o, s}$ be the count (i.e., number of occurrences) of the GO term go in species $s$ contained in our set and let $N_{g o, s}$ be the count for the whole genome. Furthermore, let $n_{s}=\sum_{g o} n_{g o, s}$ and $N_{s}=\sum_{g o} N_{g o, s}$. Assume that all species under consideration diverged at the same time and evolved independently after that (i.e. they have a starlike phylogeny). The null hypothesis of the MSGEA test is that a given GO term was not over-represented in the common ancestor. Therefore, a significant $p$-value means that the genome of the ancestor was already enriched in the GO term considered. Being conservative, assume that the null distributions of $n_{g o, s}$ are independent hypergeometrics with parameters $N_{g o, s}, n_{s}$ and $N_{s}$ in each species. The enrichment statistics $X_{g o}$ is then the sum of the normalized enrichments of $n_{g o, s}$ across species, i.e. the sum of the $z$-scores:

$$
X_{g o}=\sum_{s} \frac{n_{g o, s}-\mathrm{E}\left(n_{g o, s}\right)}{\mathrm{SD}\left(n_{g o, s}\right)} \sim \sum_{s} \frac{n_{g o, s}-N_{g o, s} n_{s} / N_{s}}{\sqrt{N_{g o, s} n_{s} / N_{s}}}
$$

where a Poisson approximation is used to define the score. Finally, the $p$ value is the probability

$$
p_{g o}=\operatorname{Prob}\left(x \geq X_{g o} \mid N_{g o, s}, n_{s}, N_{s}\right),
$$

where the distribution of $x$ follows from the hypergeometric distributions of the $n_{g o, s}$ with the above parameters.

For a single species, this test coincides with the standard one-tailed Fisher's exact test for GO enrichment, and therefore is consistent. The exact estimation of $p$-values is computationally intensive; an optimized code, written in $\mathrm{C}$, is available from the authors upon request. Since MSGEA is based on the hypothesis of independent evolution of each lineage, it can be applied only to starlike phylogenies. For the situation considered here, this assumption is met, since the "roundworm-fruit fly-zebra fish" phylogeny is 
approximately starlike. We find that a considerable fraction (30-70\%) of significant GO terms in our data set is detected by MSGEA, but not by single species enrichment.

\section{Detailed functional analysis of genes from model organisms}

To mine literature databases for functional studies in model organisms with a focus on development we employed biomaRt: we used the Bioconductor module [68] biomaRt 2.19.3 to extract information for proteins present in cluster set $L^{\prime}$. Wormbase release WS220 was queried for C. elegans proteins and ENSEMBL 75 for D. melanogaster and D. rerio proteins. We then searched the literature for experimental validations of protein function. We grouped the results in six major categories, described in Results and Discussion and summarized in supplementary Table S5. These categories represent prominent molecular functions during embryogenesis and development. Based on the retrieved annotations we assigned proteins in set $L^{\prime}$ to these categories.

\section{Cross species expression profiles}

We retrieved expression data for different developmental stages in $D$. melanogaster $[69,70]$, D. rerio [32] and C. elegans [71]. D. rerio data for adult stages were not used. The expression profiles were transformed by taking the logarithm to base 10 of the expression levels and then subtracting the $\log _{10}$ of the mean expression at each stage.

We devised the following test to find characteristic expression patterns: first, we computed Pearson's correlation coefficient between expression profiles for different genes. For each profile in each set of the genes from 85 COPs, we performed two types of tests: (i) a Mann-Whitney test on the distribution of correlations, comparing the correlation of the profile with other bilaterian genes versus the correlation with genome-wide profiles, in order to detect profiles that are more correlated with the ones of other bilaterian genes than with the rest of the genome; (ii) for each profile, we classified the remaining genes as "highly correlated" or "not highly correlated" in expression, using correlation thresholds of $r=0.5,0.7$ and 0.9 ; then, we tested for enrichment of correlated profiles among Bilaterian genes by Fisher's exact test.

A Benjamini-Hochberg correction for multiple testing was applied to the $p$-values resulting from these tests. All the significant profiles $(p<0.05)$ were 
clustered based on their correlation coefficients $r$ by complete-linkage hierarchical clustering implemented in the $\mathrm{R}$ statistics software package, using $1-r$ as distance measure and selecting clusters at height $h=0.75$. After clustering, profiles were normalized by their average expression across stages, then averaged across each cluster. The resulting profile shapes are shown in row 2 of Fig 5 .

Are expression profiles driven by gene function?

We performed a randomisation test to explore if the mean (logarithmic) expression level of COPs genes could be explained by their function. For each developmental stage we checked if expression correlates with that of randomly selected genes possessing equal or similar GO terms, i.e. we checked if genes of different age, but similar ontology, would show similar expression profiles. We performed this analysis only on set $L^{\prime}$.

For this purpose, we randomly sampled from the whole genome 100 sets with the same number of genes as are contained in set $L^{\prime}$. We did this in two different ways. (i) The first ("random-background") was a random sampling of genes from the genome. After sampling, we computed the mean normalized expression of the selected genes for each stage. We repeated this procedure 100 times, and thus obtained a distribution of normalized means, represented as boxplots (in white) in Fig 5, Fig S3 and Fig S4. (ii) The second was a "matched-function" sampling: first, for each GO term in the original set, we listed all genes with the same annotation and extracted from this list a number of random genes equal to the number of occurrences of the term; second, we pooled all the resulting lists; third, we extracted from this list a number of random genes equal to the number of genes in the original set. This way, we obtained sets of the same size and function (approximately, at least) as our original set. We applied this to the whole set $L^{\prime}$ and for all subsets corresponding to the six functional categories described above (Fig S4).

\section{Age index of proteins}

We used the phylostratigraphy from Domazet-Lošo et al. [32] for $D$. melanogaster, D. rerio and C. elegans to define two age-groups of proteins: (i) older proteins, with an origin in phylostrata 1 to 6; (ii) younger proteins, with an origin in phylostrata 8 and higher. Proteins that we identified as bilaterian-specific (i.e. the ones in our sets $A, L, M$ and $C$ ) were excluded 
636 from the two groups, irrespective of their original phylostratigraphic classifi${ }_{637}$ cation. We repeated all the analyses described in the previous sections, and 638 compared separately our COPs genes with the genome-wide background of 639 older and of younger genes (Fig 5). 


\section{Acknowledgements}

We are grateful to Peter Heger (University of Cologne) for his input on previous versions of this manuscript. We are also very thankful to Thomas Wiehe (University of Cologne) for his support in conducting this research, his input on functional profile analysis and many vibrant and fruitful discussions leading to the finished manuscript. We thank Georgios Koutsovoulos (INRA Sophia-Antipolis) and Sujai Kumar (University of Edinburgh) for help with the initial orthology clustering.

\section{References}

[1] C. R. Marschall, Explaining the Cambrian "Explosion" of Animals, Annual Review of Earth and Planetary Sciences 34: 355-384. doi:10.1146/annurev.earth.33.031504.103001.

[2] C. Nielsen, N. Scharff, D. Eibye-Jacobsen, Cladistic analyses of the animal kingdom, Biological Journal of the Linnean Society 57 (4) (1996) 385-410. doi:10.1111/j.1095-8312.1996.tb01857.x.

[3] D. Erwin, E. Davidson, The last common bilaterian ancestor., Development 129 (13) (2002) 3021-3032.

[4] M. Telford, A. Lockyer, C. Cartwright-Finch, D. Littlewood, Combined large and small subunit ribosomal RNA phylogenies support a basal position of the acoelomorph flatworms., Proc Biol Sci 270 (1519) (2003) 1077-1083. doi:10.1098/rspb.2003.2342.

[5] A. Hejnol, M. Martindale, Acoel development indicates the independent evolution of the bilaterian mouth and anus., Nature 456 (7220) (2008) 382-386. doi:10.1038/nature07309.

[6] H. Philippe, H. Brinkmann, R. Copley, L. Moroz, H. Nakano, A. Poustka, A. Wallberg, K. Peterson, M. Telford, Acoelomorph flatworms are deuterostomes related to Xenoturbella., Nature 470 (7333) (2011) 255-258. doi:10.1038/nature09676.

[7] J. Cannon, B. Vellutini, J. Smith, 3rd, F. Ronquist, U. Jondelius, A. Hejnol, Xenacoelomorpha is the sister group to Nephrozoa., Nature 530 (7588) (2016) 89-93. doi:10.1038/nature16520. 
[8] M. dos Reis, Y. Thawornwattana, K. Angelis, M. Telford, P. Donoghue, Z. Yang, Uncertainty in the Timing of Origin of Animals and the Limits of Precision in Molecular Timescales., Curr Biol 25 (22) (2015) 2939 2950. doi:10.1016/j.cub.2015.09.066.

[9] N. Irie, S. Kuratani, The developmental hourglass model: a predictor of the basic body plan?, Development 141 (24) (2014) 4649-4655. doi:10.1242/dev.107318.

[10] I. Yanai, Development and Evolution through the Lens of Global Gene Regulation., Trends Genet 34 (1) (2018) 11-20. doi:10.1016/j.tig.2017.09.011.

[11] H. Zalts, I. Yanai, Developmental constraints shape the evolution of the nematode mid-developmental transition., Nat Ecol Evol 1 (5) (2017) 113. doi:10.1038/s41559-017-0113.

[12] M. Levin, L. Anavy, A. G. Cole, E. Winter, N. Mostov, S. Khair, N. Senderovich, E. Kovalev, D. H. Silver, M. Feder, S. L. FernandezValverde, N. Nakanishi, D. Simmons, O. Simakov, T. Larsson, S.-Y. Liu, A. Jerafi-Vider, K. Yaniv, J. F. Ryan, M. Q. Martindale, J. C. Rink, D. Arendt, S. M. Degnan, B. M. Degnan, T. Hashimshony, I. Yanai, The mid-developmental transition and the evolution of animal body plans, Nature 531 (7596) (2016) 637-641.

[13] J. Liu, M. Robinson-Rechavi, Developmental Constraints on Genome Evolution in Four Bilaterian Model Species, Genome biology and evolution 10 (9) (2018) 2266-2277.

[14] D. Duboule, Temporal colinearity and the phylotypic progression: a basis for the stability of a vertebrate Bauplan and the evolution of morphologies through heterochrony., Dev Suppl (1994) 135-142.

[15] R. A. Raff, The Shape of Life, Genes, Development, and the Evolution of Animal Form, University of Chicago Press, 1996.

[16] K. E. v. Baer, Über Entwickelungsgeschichte der Thiere. Beobachtung und Reflexion ..., Bei den Gebrüdern Bornträger,, Königsberg,, 1828.

[17] M. Saina, G. Genikhovich, E. Renfer, U. Technau, BMPs and chordin regulate patterning of the directive axis in a sea anemone., 
Proc Natl Acad Sci U S A 106 (44) (2009) 18592-18597. doi:10.1073/pnas.0900151106.

[18] D. Gauchat, F. Mazet, C. Berney, M. Schummer, S. Kreger, J. Pawlowski, B. Galliot, Evolution of Antp-class genes and differential expression of Hydra Hox/paraHox genes in anterior patterning., Proc Natl Acad Sci U S A 97 (9) (2000) 4493-4498.

[19] C. Sinigaglia, H. Busengdal, L. Leclere, U. Technau, F. Rentzsch, The bilaterian head patterning gene six $3 / 6$ controls aboral domain development in a cnidarian., PLoS Biol 11 (2) (2013) e1001488. doi:10.1371/journal.pbio.1001488.

[20] H. Sun, A. Rodin, Y. Zhou, D. Dickinson, D. Harper, D. HewettEmmett, W. Li, Evolution of paired domains: isolation and sequencing of jellyfish and hydra Pax genes related to Pax-5 and Pax-6., Proc Natl Acad Sci U S A 94 (10) (1997) 5156-5161.

[21] O. Simakov, F. Marletaz, S. Cho, E. Edsinger-Gonzales, P. Havlak, U. Hellsten, D. Kuo, T. Larsson, J. Lv, D. Arendt, R. Savage, K. Osoegawa, P. de Jong, J. Grimwood, J. Chapman, H. Shapiro, A. Aerts, R. Otillar, A. Terry, J. Boore, I. Grigoriev, D. Lindberg, E. Seaver, D. Weisblat, N. Putnam, D. Rokhsar, Insights into bilaterian evolution from three spiralian genomes., Nature 493 (7433) (2013) 526-531. doi:10.1038/nature11696.

[22] L. Moroz, K. Kocot, M. Citarella, S. Dosung, T. Norekian, I. Povolotskaya, A. Grigorenko, C. Dailey, E. Berezikov, K. Buckley, A. Ptitsyn, D. Reshetov, K. Mukherjee, T. Moroz, Y. Bobkova, F. Yu, V. Kapitonov, J. Jurka, Y. Bobkov, J. Swore, D. Girardo, A. Fodor, F. Gusev, R. Sanford, R. Bruders, E. Kittler, C. Mills, J. Rast, R. Derelle, V. Solovyev, F. Kondrashov, B. Swalla, J. Sweedler, E. Rogaev, K. Halanych, A. Kohn, The ctenophore genome and the evolutionary origins of neural systems., Nature 510 (7503) (2014) 109-114. doi:10.1038/nature13400.

[23] M. Srivastava, O. Simakov, J. Chapman, B. Fahey, M. Gauthier, T. Mitros, G. Richards, C. Conaco, M. Dacre, U. Hellsten, C. Larroux, N. Putnam, M. Stanke, M. Adamska, A. Darling, S. Degnan, 
T. Oakley, D. Plachetzki, Y. Zhai, M. Adamski, A. Calcino, S. Cummins, D. Goodstein, C. Harris, D. Jackson, S. Leys, S. Shu, B. Woodcroft, M. Vervoort, K. Kosik, G. Manning, B. Degnan, D. Rokhsar, The Amphimedon queenslandica genome and the evolution of animal complexity., Nature 466 (7307) (2010) 720-726. doi:10.1038/nature09201.

[24] N. Putnam, M. Srivastava, U. Hellsten, B. Dirks, J. Chapman, A. Salamov, A. Terry, H. Shapiro, E. Lindquist, V. Kapitonov, J. Jurka, G. Genikhovich, I. Grigoriev, S. Lucas, R. Steele, J. Finnerty, U. Technau, M. Martindale, D. Rokhsar, Sea anemone genome reveals ancestral eumetazoan gene repertoire and genomic organization., Science 317 (5834) (2007) 86-94. doi:10.1126/science.1139158.

[25] O. Simakov, T. Kawashima, F. Marletaz, J. Jenkins, R. Koyanagi, T. Mitros, K. Hisata, J. Bredeson, E. Shoguchi, F. Gyoja, J.-X. Yue, Y.C. Chen, R. M. J. Freeman, A. Sasaki, T. Hikosaka-Katayama, A. Sato, M. Fujie, K. W. Baughman, J. Levine, P. Gonzalez, C. Cameron, J. H. Fritzenwanker, A. M. Pani, H. Goto, M. Kanda, N. Arakaki, S. Yamasaki, J. Qu, A. Cree, Y. Ding, H. H. Dinh, S. Dugan, M. Holder, S. N. Jhangiani, C. L. Kovar, S. L. Lee, L. R. Lewis, D. Morton, L. V. Nazareth, G. Okwuonu, J. Santibanez, R. Chen, S. Richards, D. M. Muzny, A. Gillis, L. Peshkin, M. Wu, T. Humphreys, Y.-H. Su, N. H. Putnam, J. Schmutz, A. Fujiyama, J.-K. Yu, K. Tagawa, K. C. Worley, R. A. Gibbs, M. W. Kirschner, C. J. Lowe, N. Satoh, D. S. Rokhsar, J. Gerhart, Hemichordate genomes and deuterostome origins., Nature 527 (7579) (2015) 459-465. doi:10.1038/nature16150.

[26] L. Li, C. Stoeckert, Jr., D. Roos, OrthoMCL: identification of ortholog groups for eukaryotic genomes., Genome Res 13 (9) (2003) 2178-2189. doi:10.1101/gr.1224503.

[27] J. Taylor, I. Braasch, T. Frickey, A. Meyer, Y. Van de Peer, Genome duplication, a trait shared by 22000 species of ray-finned fish., Genome Res 13 (3) (2003) 382-390. doi:10.1101/gr.640303.

[28] A. Siepel, G. Bejerano, J. Pedersen, A. Hinrichs, M. Hou, K. Rosenbloom, H. Clawson, J. Spieth, L. Hillier, S. Richards, G. Weinstock, R. Wilson, R. Gibbs, W. Kent, W. Miller, D. Haussler, Evolutionarily conserved elements in vertebrate, insect, worm, and yeast genomes., Genome Res 15 (8) (2005) 1034-1050. doi:10.1101/gr.3715005. 
[29] M. Gerstein, J. Rozowsky, K. Yan, D. Wang, C. Cheng, J. Brown, C. Davis, L. Hillier, C. Sisu, J. Li, B. Pei, A. Harmanci, M. Duff, S. Djebali, R. Alexander, B. Alver, R. Auerbach, K. Bell, P. Bickel, M. Boeck, N. Boley, B. Booth, L. Cherbas, P. Cherbas, C. Di, A. Dobin, J. Drenkow, B. Ewing, G. Fang, M. Fastuca, E. Feingold, A. Frankish, G. Gao, P. Good, R. Guigo, A. Hammonds, J. Harrow, R. Hoskins, C. Howald, L. Hu, H. Huang, T. Hubbard, C. Huynh, S. Jha, D. Kasper, M. Kato, T. Kaufman, R. Kitchen, E. Ladewig, J. Lagarde, E. Lai, J. Leng, Z. Lu, M. MacCoss, G. May, R. McWhirter, G. Merrihew, D. Miller, A. Mortazavi, R. Murad, B. Oliver, S. Olson, P. Park, M. Pazin, N. Perrimon, D. Pervouchine, V. Reinke, A. Reymond, G. Robinson, A. Samsonova, G. Saunders, F. Schlesinger, A. Sethi, F. Slack, W. Spencer, M. Stoiber, P. Strasbourger, A. Tanzer, O. Thompson, K. Wan, G. Wang, H. Wang, K. Watkins, J. Wen, K. Wen, C. Xue, L. Yang, K. Yip, C. Zaleski, Y. Zhang, H. Zheng, S. Brenner, B. Graveley, S. Celniker, T. Gingeras, R. Waterston, Comparative analysis of the transcriptome across distant species., Nature 512 (7515) (2014) 445-448. doi:10.1038/nature13424.

[30] M. Ninova, M. Ronshaugen, S. Griffiths-Jones, Conserved temporal patterns of microRNA expression in Drosophila support a developmental hourglass model., Genome Biol Evol 6 (9) (2014) 2459-2467. doi:10.1093/gbe/evu183.

[31] P. H. Schiffer, A. L. Polsky, A. G. Cole, J. I. R. Camps, M. Kroiher, D. H. Silver, V. Grishkevich, L. Anavy, G. Koutsovoulos, T. Hashimshony, I. Yanai, The gene regulatory program of Acrobeloides nanus reveals conservation of phylum-specific expression., Proceedings of the National Academy of Sciences 1 (17) (2018) 201720817-4464.

[32] T. Domazet-Loso, D. Tautz, Phylostratigraphic tracking of cancer genes suggests a link to the emergence of multicellularity in metazoa., BMC Biol 8 (2010) 66. doi:10.1186/1741-7007-8-66.

[33] M. Quint, H. Drost, A. Gabel, K. Ullrich, M. Bonn, I. Grosse, A transcriptomic hourglass in plant embryogenesis., Nature 490 (7418) (2012) 98-101. doi:10.1038/nature11394.

[34] X. Cheng, J. Hui, Y. Lee, P. Wan Law, H. Kwan, A "develop- 
mental hourglass" in fungi., Mol Biol Evol 32 (6) (2015) 1556-1566. doi:10.1093/molbev/msv047.

[35] T. Domazet-Loso, J. Brajkovic, D. Tautz, A phylostratigraphy approach to uncover the genomic history of major adaptations in metazoan lineages, Trends in Genetics 23 (11) (2007) 533 - 539. doi:http://dx.doi.org/10.1016/j.tig.2007.08.014.

[36] M. Sestak, T. Domazet-Loso, Phylostratigraphic profiles in zebrafish uncover chordate origins of the vertebrate brain., Mol Biol Evol 32 (2) (2015) 299-312. doi:10.1093/molbev/msu319.

[37] P. Heger, B. Marin, M. Bartkuhn, E. Schierenberg, T. Wiehe, The chromatin insulator CTCF and the emergence of metazoan diversity., Proc Natl Acad Sci U S A 109 (43) (2012) 17507-17512. doi:10.1073/pnas.1111941109.

[38] E. Koonin, Comparative genomics, minimal gene-sets and the last universal common ancestor., Nat Rev Microbiol 1 (2) (2003) 127-136. doi:10.1038/nrmicro751.

[39] D. M. Emms, S. Kelly, OrthoFinder: solving fundamental biases in whole genome comparisons dramatically improves orthogroup inference accuracy 16 (1) (2015) E9-13. doi:10.1186/s13059-015-0721-2.

[40] A. Aboobaker, M. Blaxter, Hox Gene Loss during Dynamic Evolution of the Nematode Cluster., Curr Biol 13 (1) (2003) 37-40.

[41] A. Conesa, S. Götz, J. M. García-Gómez, J. Terol, M. Talón, M. Robles, Blast2GO: a universal tool for annotation, visualization and analysis in functional genomics research., Bioinformatics (18) 3674-3676. doi:10.1093/bioinformatics/bti610.

[42] J. M. Martín-Durán, K. Pang, A. Børve, H. S. Lê, A. Furu, J. T. Cannon, U. Jondelius, A. Hejnol, Convergent evolution of bilaterian nerve cords, Nature 553 (7686) (2018) 45-50.

[43] J. Li, H. Huang, P. Bickel, S. Brenner, Comparison of D. melanogaster and $C$. elegans developmental stages, tissues, and cells by modENCODE RNA-seq data., Genome Res 24 (7) (2014) 1086-1101. doi:10.1101/gr.170100.113. 
[44] C. Kimmel, W. Ballard, S. Kimmel, B. Ullmann, T. Schilling, Stages of embryonic development of the zebrafish., Dev Dyn 203 (3) (1995) 253-310. doi:10.1002/aja.1002030302.

[45] P. Schiffer, M. Kroiher, C. Kraus, G. Koutsovoulos, S. Kumar, J. Camps, N. Nsah, D. Stappert, K. Morris, P. Heger, J. Altmuller, P. Frommolt, P. Nurnberg, W. Thomas, M. Blaxter, E. Schierenberg, The genome of Romanomermis culicivorax: revealing fundamental changes in the core developmental genetic toolkit in Nematoda., BMC Genomics 14 (2013) 923. doi:10.1186/1471-2164-14-923.

[46] E. Davidson, D. Erwin, Gene regulatory networks and the evolution of animal body plans., Science 311 (5762) (2006) 796-800. doi:10.1126/science.1113832.

[47] M. Macchietto, D. Angdembey, N. Heidarpour, L. Serra, B. Rodriguez, N. El-Ali, A. Mortazavi, Comparative Transcriptomics of Steinernema and Caenorhabditis Single Embryos Reveals Orthologous Gene Expression Convergence during Late Embryogenesis., Genome biology and evolution 9 (10) (2017) 2681-2696.

[48] M. Schwaiger, A. Schonauer, A. Rendeiro, C. Pribitzer, A. Schauer, A. Gilles, J. Schinko, E. Renfer, D. Fredman, U. Technau, Evolutionary conservation of the eumetazoan gene regulatory landscape., Genome Res 24 (4) (2014) 639-650. doi:10.1101/gr.162529.113.

[49] A. Sebé-Pedrós, E. Chomsky, K. Pang, D. Lara-Astiaso, F. Gaiti, Z. Mukamel, I. Amit, A. Hejnol, B. M. Degnan, A. Tanay, Early metazoan cell type diversity and the evolution of multicellular gene regulation, Nature Ecology \& Evolution (2018) 1-17.

[50] T. Gabaldón, E. V. Koonin, Functional and evolutionary implications of gene orthology, Nature Publishing Group 14 (5) (2013) 360-366. doi: $10.1038 / \operatorname{nrg} 3456$.

[51] N. L. Nehrt, W. T. Clark, P. Radivojac, M. W. Hahn, Testing the Ortholog Conjecture with Comparative Functional Genomic Data from Mammals, PLoS computational biology 7 (6) (2011) e1002073-10. doi:10.1371/journal.pcbi.1002073. 
[52] A. M. Altenhoff, R. A. Studer, M. Robinson-Rechavi, C. Dessimoz, Resolving the Ortholog Conjecture: Orthologs Tend to Be Weakly, but Significantly, More Similar in Function than Paralogs, PLoS computational biology 8 (5) (2012) e1002514-10. doi:10.1371/journal.pcbi.1002514. URL https://dx.plos.org/10.1371/journal.pcbi.1002514

[53] N. Kryuchkova-Mostacci, M. Robinson-Rechavi, Tissue-Specificity of Gene Expression Diverges Slowly between Orthologs, and Rapidly between Paralogs, PLoS computational biology 12 (12) (2016) e100527413. doi:10.1371/journal.pcbi.1005274. URL https://dx.plos.org/10.1371/journal.pcbi.1005274

[54] T. Begum, M. Robinson-Rechavi, Phylogenetic comparative methods are problematic when applied to gene trees with speciation and duplication nodes: correcting for biases in testing the ortholog conjecture (2019) 1-37doi:10.1101/719336. URL http://biorxiv.org/lookup/doi/10.1101/719336

[55] C. E. Konikoff, T. L. Karr, M. McCutchan, S. J. Newfeld, S. Kumar, Comparison of embryonic expression within multigene families using the flyexpress discovery platform reveals more spatial than temporal divergence, Developmental Dynamics 241 (1) (2011) 150-160. doi:10.1002/dvdy.22749.

[56] M. Srivastava, E. Begovic, J. Chapman, N. Putnam, U. Hellsten, T. Kawashima, A. Kuo, T. Mitros, A. Salamov, M. Carpenter, A. Signorovitch, M. Moreno, K. Kamm, J. Grimwood, J. Schmutz, H. Shapiro, I. Grigoriev, L. Buss, B. Schierwater, S. Dellaporta, D. Rokhsar, The Trichoplax genome and the nature of placozoans., Nature 454 (7207) (2008) 955-960. doi:10.1038/nature07191.

[57] E. Zdobnov, R. Apweiler, InterProScan-an integration platform for the signature-recognition methods in InterPro., Bioinformatics 17 (9) (2001) $847-848$.

[58] M. Ashburner, C. Ball, J. Blake, D. Botstein, H. Butler, J. Cherry, A. Davis, K. Dolinski, S. Dwight, J. Eppig, M. Harris, D. Hill, L. IsselTarver, A. Kasarskis, S. Lewis, J. Matese, J. Richardson, M. Ringwald, 
G. Rubin, G. Sherlock, Gene ontology: tool for the unification of biology. The Gene Ontology Consortium., Nat Genet 25 (1) (2000) 25-29. doi:10.1038/75556.

[59] A. Alexeyenko, I. Tamas, G. Liu, E. Sonnhammer, Automatic clustering of orthologs and inparalogs shared by multiple proteomes., Bioinformatics 22 (14) (2006) e9-15. doi:10.1093/bioinformatics/btl213.

[60] D. Shaye, I. Greenwald, OrthoList: a compendium of C. elegans genes with human orthologs., PLoS One 6 (5) (2011) e20085. doi:10.1371/journal.pone.0020085.

[61] T. Hulsen, M. Huynen, J. de Vlieg, P. Groenen, Benchmarking ortholog identification methods using functional genomics data., Genome Biol 7 (4) (2006) R31. doi:10.1186/gb-2006-7-4-r31.

[62] B. Buchfink, C. Xie, D. H. Huson, Fast and sensitive protein alignment using DIAMOND 12 (1) (2014) 59-60. doi:10.1038/nmeth.3176.

URL http://www . nature.com/doifinder/10.1038/nmeth.3176

[63] G. Moreno-Hagelsieb, K. Latimer, Choosing BLAST options for better detection of orthologs as reciprocal best hits. 24 (3) (2008) 319-324. doi:10.1093/bioinformatics/btm585.

[64] C. Dessimoz, G. Cannarozzi, M. Gil, D. Margadant, A. Roth, A. Schneider, G. H. Gonnet, OMA, A Comprehensive, Automated Project for the Identification of Orthologs from Complete Genome Data: Introduction and First Achievements, in: Comparative Genomics, Springer, Berlin, Heidelberg, Berlin, Heidelberg, 2005, pp. 61-72. doi:10.1007/11554714_6.

[65] F. Sievers, A. Wilm, D. Dineen, T. J. Gibson, K. Karplus, W. Li, R. Lopez, H. McWilliam, M. Remmert, J. Söding, J. D. Thompson, D. G. Higgins, Fast, scalable generation of high-quality protein multiple sequence alignments using Clustal Omega., Molecular systems biology 7 (1) (2011) 539-539. doi:10.1038/msb.2011.75.

[66] L.-T. Nguyen, H. A. Schmidt, A. von Haeseler, B. Q. Minh, IQ-TREE: a fast and effective stochastic algorithm for estimating maximum-likelihood phylogenies., Mol Biol Evol 32 (1) (2015) 268-274. doi:10.1093/molbev/msu300. 
[67] Y. H. Yoav Benjamini, Controlling the False Discovery Rate: A Practical and Powerful Approach to Multiple Testing, Journal of the Royal Statistical Society. Series B (Methodological) 57 (1) (1995) 289-300.

[68] J. Guberman, J. Ai, O. Arnaiz, J. Baran, A. Blake, R. Baldock, C. Chelala, D. Croft, A. Cros, R. Cutts, A. Di Genova, S. Forbes, T. Fujisawa, E. Gadaleta, D. Goodstein, G. Gundem, B. Haggarty, S. Haider, M. Hall, T. Harris, R. Haw, S. Hu, S. Hubbard, J. Hsu, V. Iyer, P. Jones, T. Katayama, R. Kinsella, L. Kong, D. Lawson, Y. Liang, N. Lopez-Bigas, J. Luo, M. Lush, J. Mason, F. Moreews, N. Ndegwa, D. Oakley, C. Perez-Llamas, M. Primig, E. Rivkin, S. Rosanoff, R. Shepherd, R. Simon, B. Skarnes, D. Smedley, L. Sperling, W. Spooner, P. Stevenson, K. Stone, J. Teague, J. Wang, J. Wang, B. Whitty, D. Wong, M. Wong-Erasmus, L. Yao, K. Youens-Clark, C. Yung, J. Zhang, A. Kasprzyk, BioMart Central Portal: an open database network for the biological community., Database (Oxford) 2011 (2011) bar041. doi:10.1093/database/bar041.

[69] B. Graveley, A. Brooks, J. Carlson, M. Duff, J. Landolin, L. Yang, C. Artieri, M. van Baren, N. Boley, B. Booth, J. Brown, L. Cherbas, C. Davis, A. Dobin, R. Li, W. Lin, J. Malone, N. Mattiuzzo, D. Miller, D. Sturgill, B. Tuch, C. Zaleski, D. Zhang, M. Blanchette, S. Dudoit, B. Eads, R. Green, A. Hammonds, L. Jiang, P. Kapranov, L. Langton, N. Perrimon, J. Sandler, K. Wan, A. Willingham, Y. Zhang, Y. Zou, J. Andrews, P. Bickel, S. Brenner, M. Brent, P. Cherbas, T. Gingeras, R. Hoskins, T. Kaufman, B. Oliver, S. Celniker, The developmental transcriptome of Drosophila melanogaster., Nature 471 (7339) (2011) 473-479. doi:10.1038/nature09715.

[70] L. Cherbas, A. Willingham, D. Zhang, L. Yang, Y. Zou, B. Eads, J. Carlson, J. Landolin, P. Kapranov, J. Dumais, A. Samsonova, J. Choi, J. Roberts, C. Davis, H. Tang, M. van Baren, S. Ghosh, A. Dobin, K. Bell, W. Lin, L. Langton, M. Duff, A. Tenney, C. Zaleski, M. Brent, R. Hoskins, T. Kaufman, J. Andrews, B. Graveley, N. Perrimon, S. Celniker, T. Gingeras, P. Cherbas, The transcriptional diversity of 25 Drosophila cell lines., Genome Res 21 (2) (2011) 301-314. doi:10.1101/gr.112961.110.

[71] M. Levin, T. Hashimshony, F. Wagner, I. Yanai, Developmental mile- 
bioRxiv preprint doi: https://doi.org/10.1101/786723; this version posted September 30, 2019. The copyright holder for this preprint (which was not certified by peer review) is the author/funder, who has granted bioRxiv a license to display the preprint in perpetuity. It is made available under aCC-BY-NC-ND 4.0 International license.

968 stones punctuate gene expression in the Caenorhabditis embryo., Dev ${ }_{969} \quad$ Cell $22(5)(2012)$ 1101-1108. doi:10.1016/j.devcel.2012.04.004. 


\section{Figures}
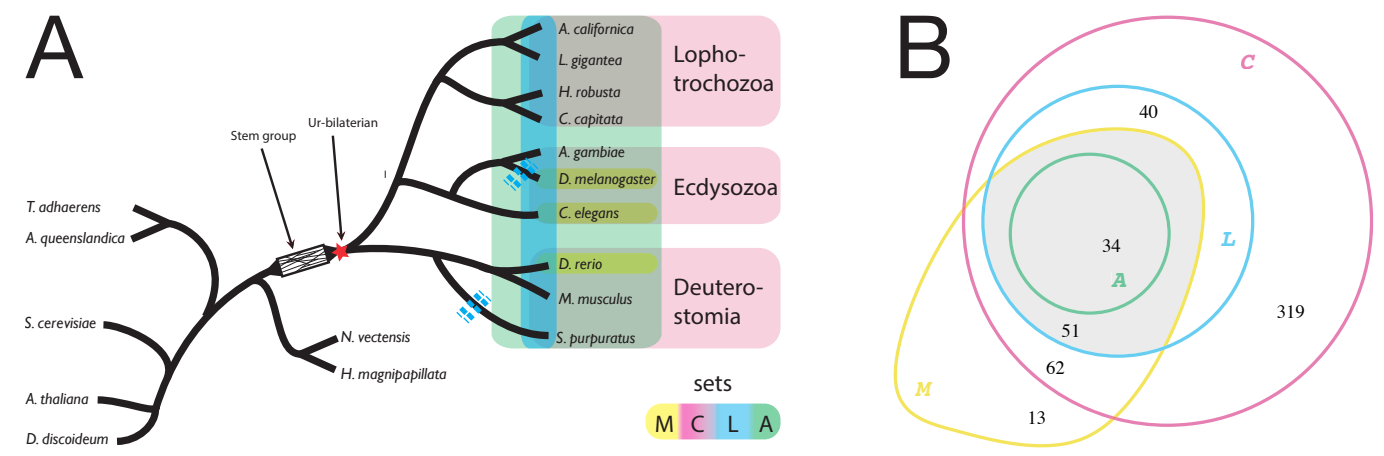

Figure 1: A: Sketch of the genealogical relationship between the clades of Lophotrochozoa, Ecdysozoa and Deuterostomia and the species included in our analysis (Table 1). Legend: $M$ - all model species are represented in a cluster; $C$ - all clades are represented; $L-$ at most one loss event along the tree (see supplementary Table $\mathrm{S} 1$ ); $A$ - all of nine bilaterian species are represented. Sketch of the 'ur-bilaterion' after [4]. Blue dashed lines indicate examples of gene loss events. B: Absolute numbers of clusters of orthologous proteins (COPs) in the four sets. Gray region: detailed analyses are performed for 85 COPs in set $L^{\prime}=L \cap M$. 
Drosophila

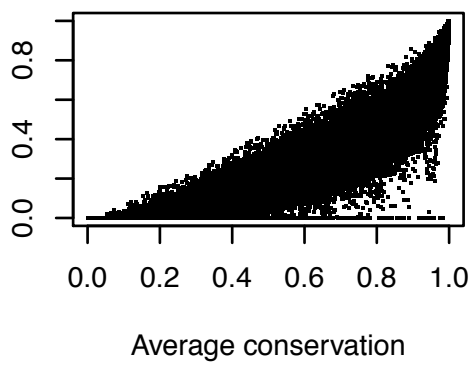

Average conservation

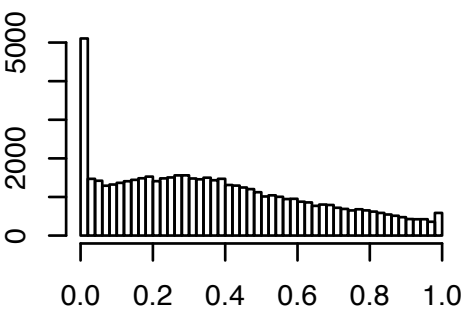

Fraction of very conserved sites

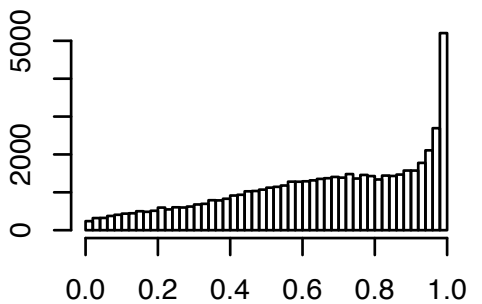

Average conservation
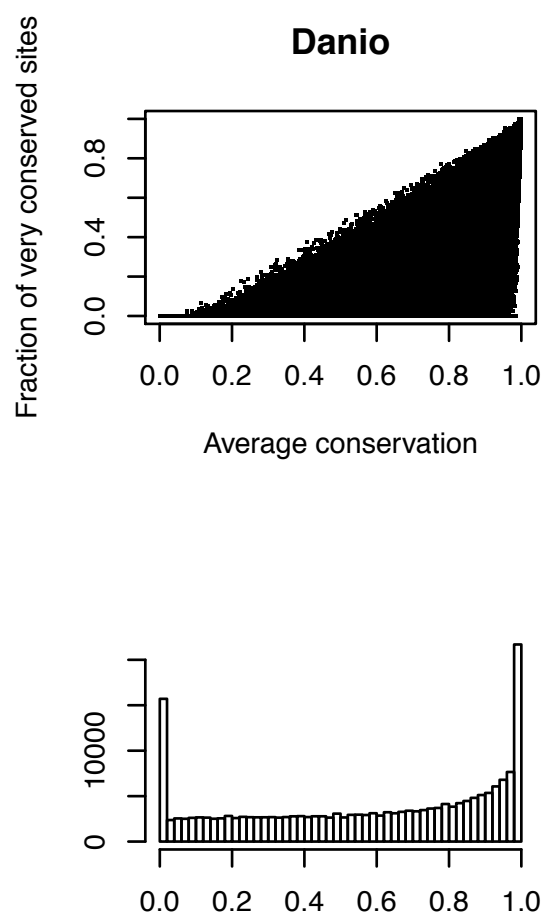

Fraction of very conserved sites

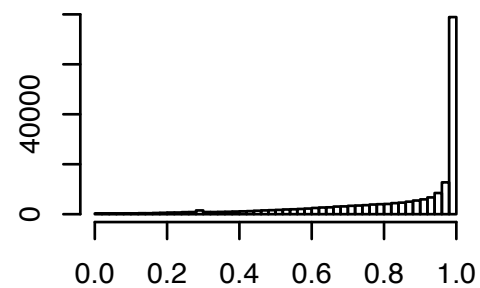

Average conservation
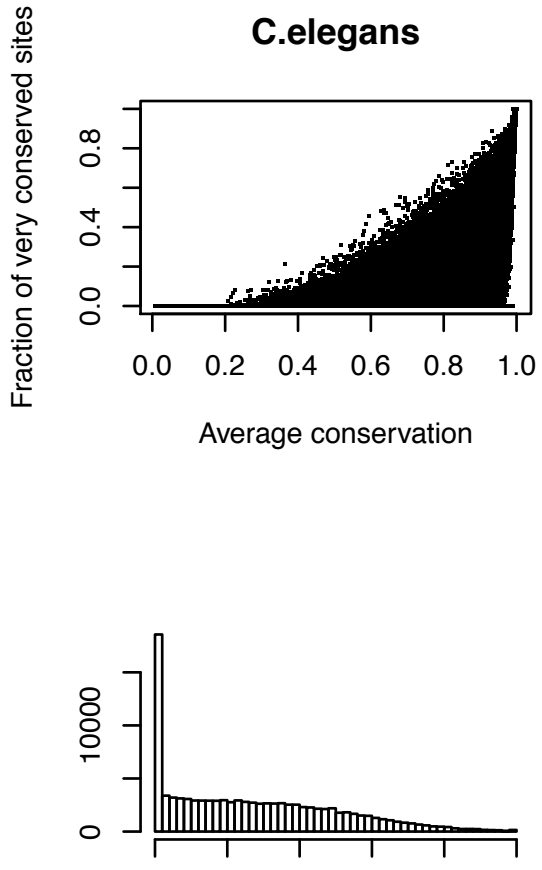

$\begin{array}{llllll}0.0 & 0.2 & 0.4 & 0.6 & 0.8 & 1.0\end{array}$

Fraction of very conserved sites

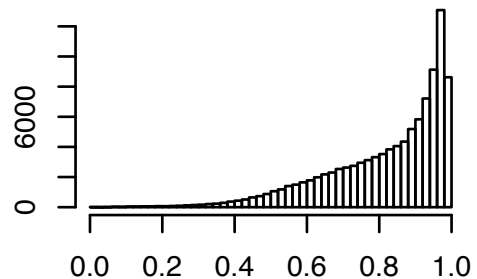

Average conservation

Figure 2: Conservation scores for ortholgous exons from PhastCons analysis. For each species, we show the correlation between scores (top), the distribution of the fraction of very conserved sites (middle), and of the average conservation score (bottom) among exons. 


\section{MSGEA: number of significant GO terms in set L'}
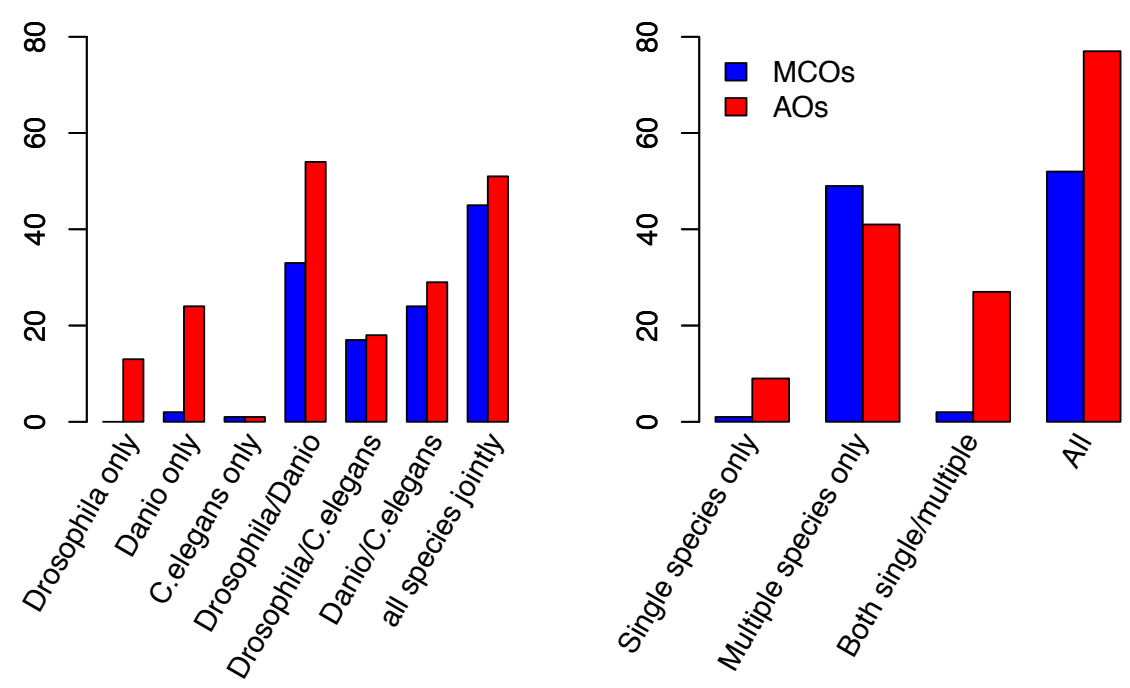

Figure 3: Number of significant GO terms identified with MSGEA in set $L^{\prime}(p<0.05)$. Red histograms: considering all orthologs (AO) from a cluster; blue histograms: only the most conserved orthologs (MCO) are considered in the MSGEA analysis. Left: number of significant terms found for each single- or multi-species enrichment analysis. Right: number of significant terms found only in single/multiple-species enrichment analyses and in both kind of analyses. The large amount of GO terms found only in multiple species analysis illustrates the power of MSGEA (see also Fig S1). 


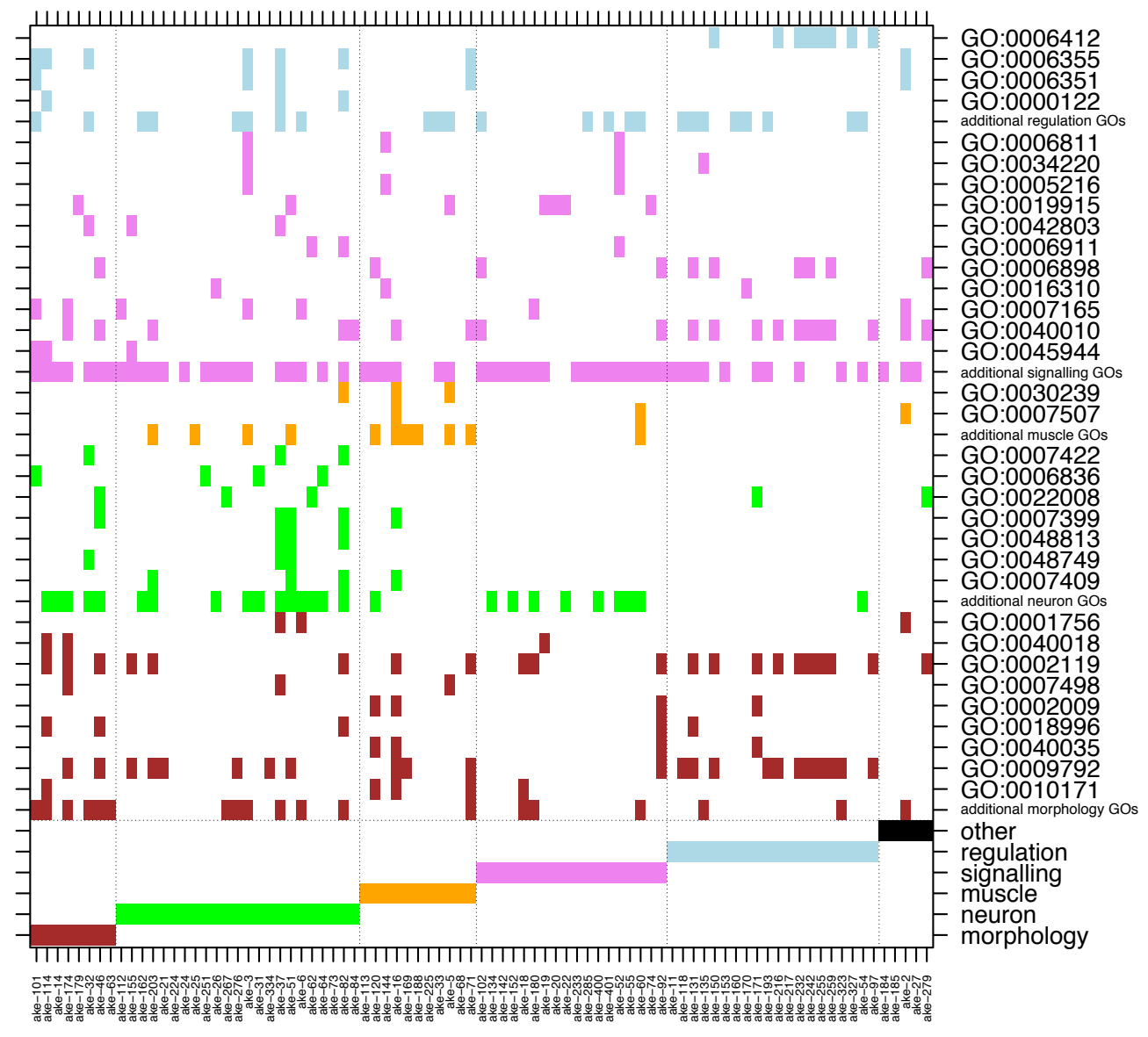

Figure 4: Significant GO terms identified in set $L^{\prime}$ by applying MSGEA (see text). The upper part of the figure shows the GO terms which occur in each COP ( $x$-axis). Those terms which occur at least in three COPs are spelled out with their GO-ID, otherwise they are collected in the lines 'additional ... GOs'. Only non-generic GO terms of level 4 and higher are recorded. The bottom six rows show the assignment of COPs to functional classes based on human-curated literature mining. As can be seen from the color code, automatic GO and human-curated functional assignments do not always coincide. 

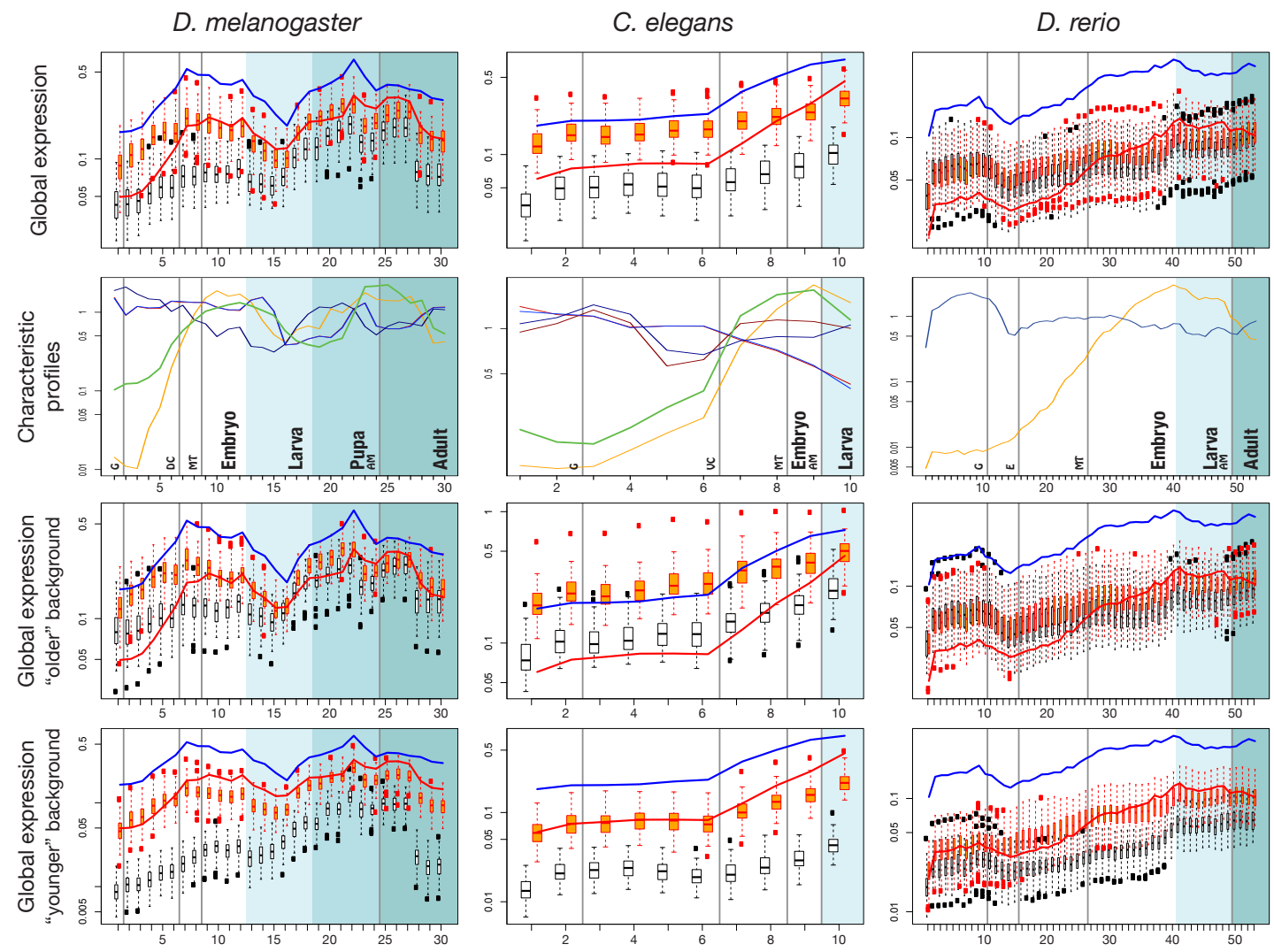

Figure 5: Expression profiles of different species. Top row: average (global) expression profiles for genes collected in set $L^{\prime}$. Red line: pooled profiles for 'all orthologs and paralogs' (set 'AO'). Blue line: profiles for the 'most conserved orthologs' (set 'MCO'). The white and orange boxplots show the 'random' and 'matched-function' expression backgrounds, respectively (see text). Second row: Characteristic expression profiles. Different colors represent different clusters. Darker and lighter colors represent profiles which are similar among species. Bottom rows: distributions shown in boxplots are calculated from genes which are younger (phylostrata $\geq 7$ according to the terminology of [32]) or older (phylostrata $<7$ ) than bilateria. Background shading (from white to dark grey) represents developmental stages embryo, larva, pupa (fly only) and adult. For a detailed explanation of the developmental stages see supplementary Tables S2 and S3. Meaning of labels in row 2: G - gastrulation; DC - dorsal closure; MT - first muscle twitching; AM - transition to adult morphology; VC - ventral closure; E - 100\% epiboly 


\section{Tables}

Table 1: Species considered to contrast bilaterian and non-bilaterian protein repertoires

\begin{tabular}{|c|c|c|c|c|c|}
\hline species & $\begin{array}{c}\text { data } \\
\text { source }\end{array}$ & $\begin{array}{l}\text { no. proteins } \\
\text { in database }\end{array}$ & $\begin{array}{l}\text { no. proteins } \\
\text { in all COPs }\end{array}$ & $\begin{array}{c}\text { no. COPs } \\
\text { w/ } \text { species }^{1}\end{array}$ & $\begin{array}{c}\text { no. proteins } \\
\text { per } \mathrm{COP}^{2}\end{array}$ \\
\hline \multicolumn{6}{|c|}{ Bilateria } \\
\hline \multicolumn{6}{|c|}{ Deuterostomia } \\
\hline Danio rerio & Ensembl & 41693 & 1190 & 428 & 2.78 \\
\hline Strongylocentrotus purpuratus & NCBI & 42420 & 652 & 260 & 2.51 \\
\hline Mus musculus & Ensembl & 40732 & 867 & 357 & 2.43 \\
\hline \multicolumn{6}{|c|}{ Ecdysozoa } \\
\hline Anopheles gambiae & Ensembl & 13133 & 380 & 307 & 1.24 \\
\hline Drosophila melanogaster & FlyBase & 23849 & 989 & 392 & 2.52 \\
\hline Caenorhabditis elegans & WormBase & 25634 & 602 & 291 & 2.07 \\
\hline \multicolumn{6}{|c|}{ Lophotrochozoa } \\
\hline Aplysia californica & NCBI & 1093 & 15 & 7 & 2.14 \\
\hline Capitella teleta & JGI & 32415 & 642 & 376 & 1.71 \\
\hline Helobdella robusta & JGI & 23432 & 420 & 325 & 1.29 \\
\hline Lottia gigantea & JGI & 23851 & 492 & 337 & 1.46 \\
\hline \multicolumn{6}{|c|}{ Non-Bilateria } \\
\hline Amphimedon queenslandica & NCBI & 9908 & & & \\
\hline Arabidopsis thaliana & TAIR & 28952 & & & \\
\hline Dictyostelium discoideum & Dictybase & 13426 & & & \\
\hline Hydra magnipapillata & NCBI & 17563 & & & \\
\hline Nematostella vectensis & JGI & 27273 & & & \\
\hline Saccharomyces cerevisiae & SGD & 6692 & & & \\
\hline Trichoplax adhaerens & JGI & 11520 & & & \\
\hline
\end{tabular}

2: average number of paralogs within a COP (number of proteins / number of COPs) 


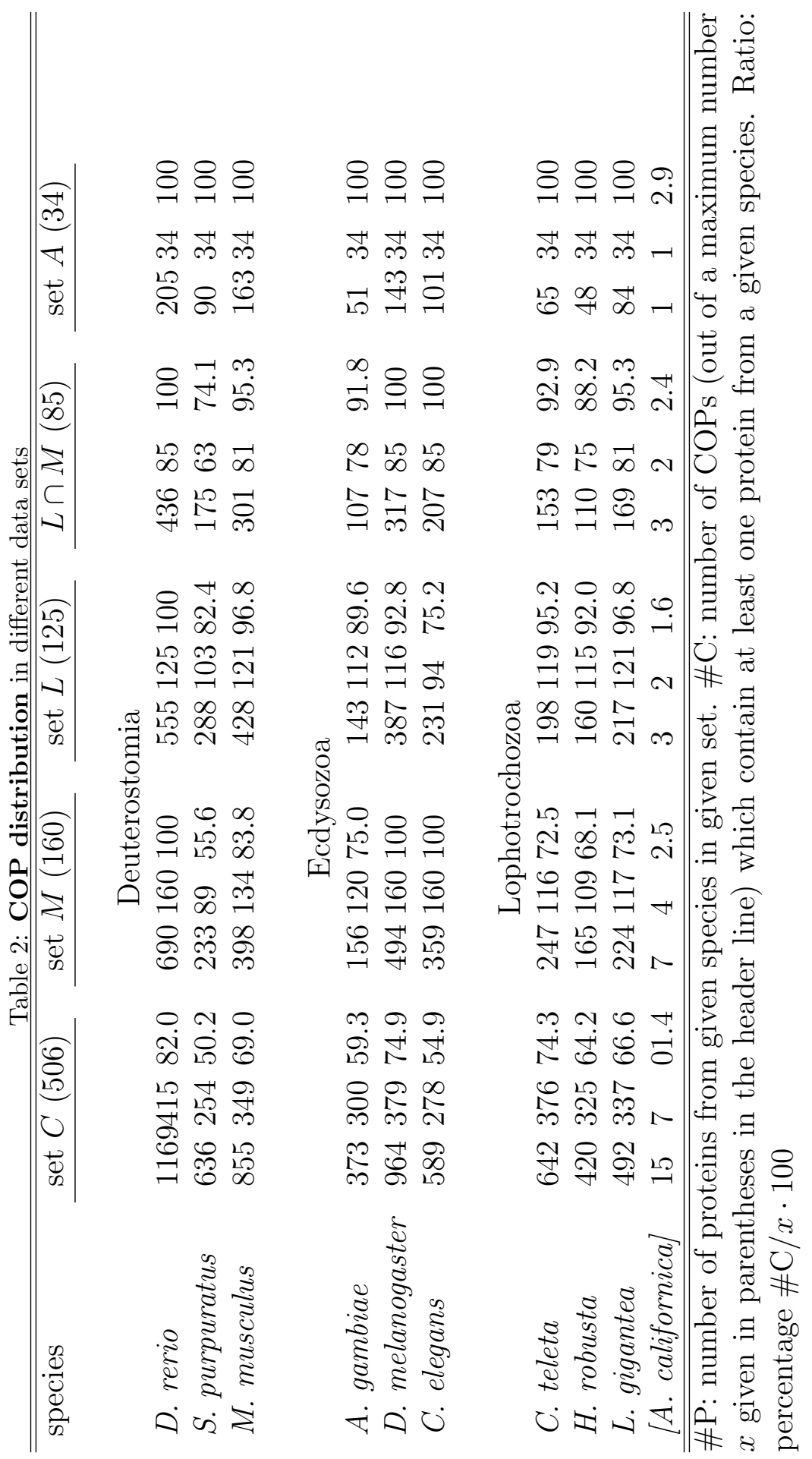


Supplementary Figures and Tables
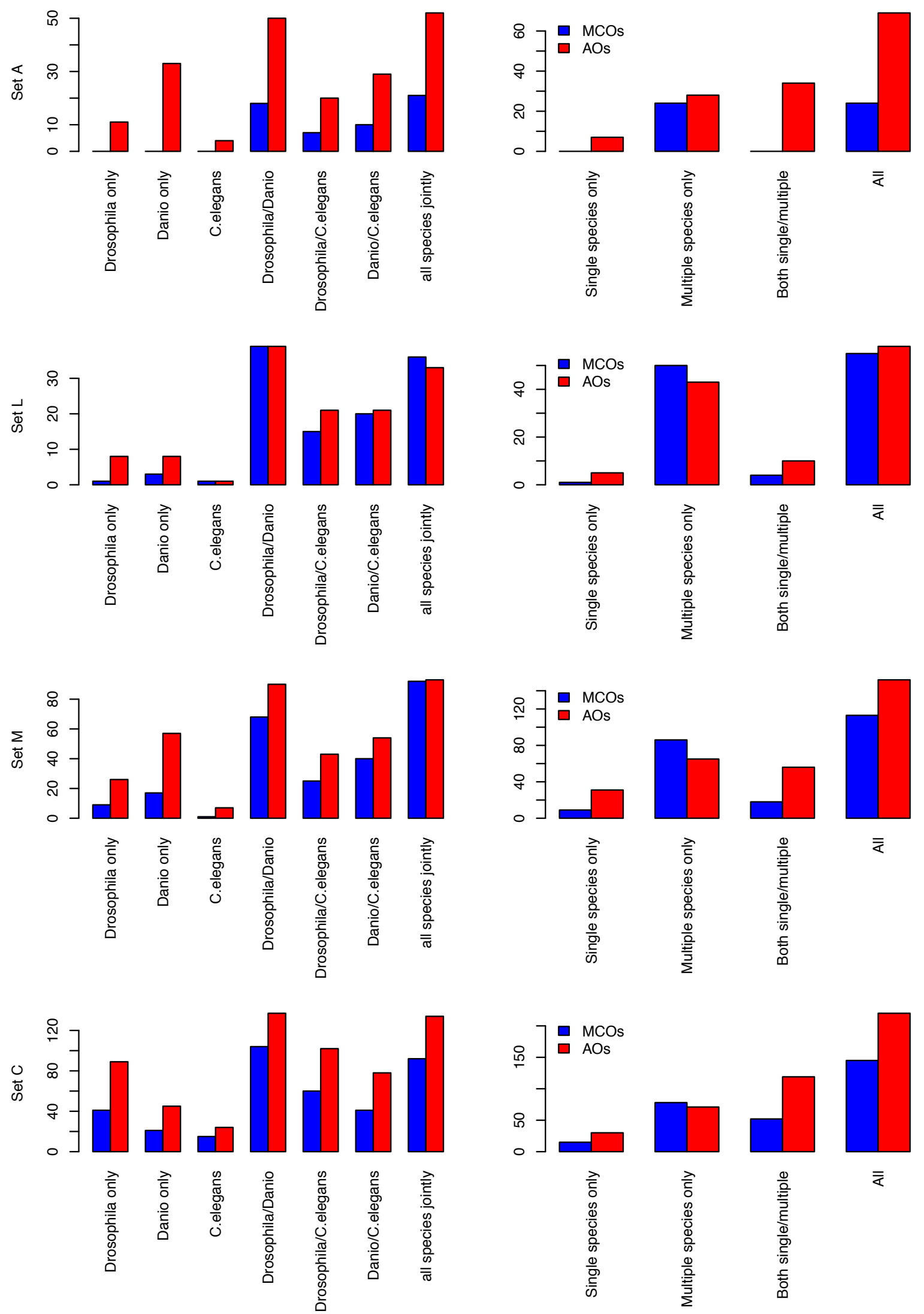

Figure S1. Supplementary Figure related to Figure 3. MSGEA vs. single species enrichment analysis for clusters of orthologous proteins (COPs). Number of significant terms shown for each of the four sets $A, L$, $M$, and $C$ (cf. Fig 3). 


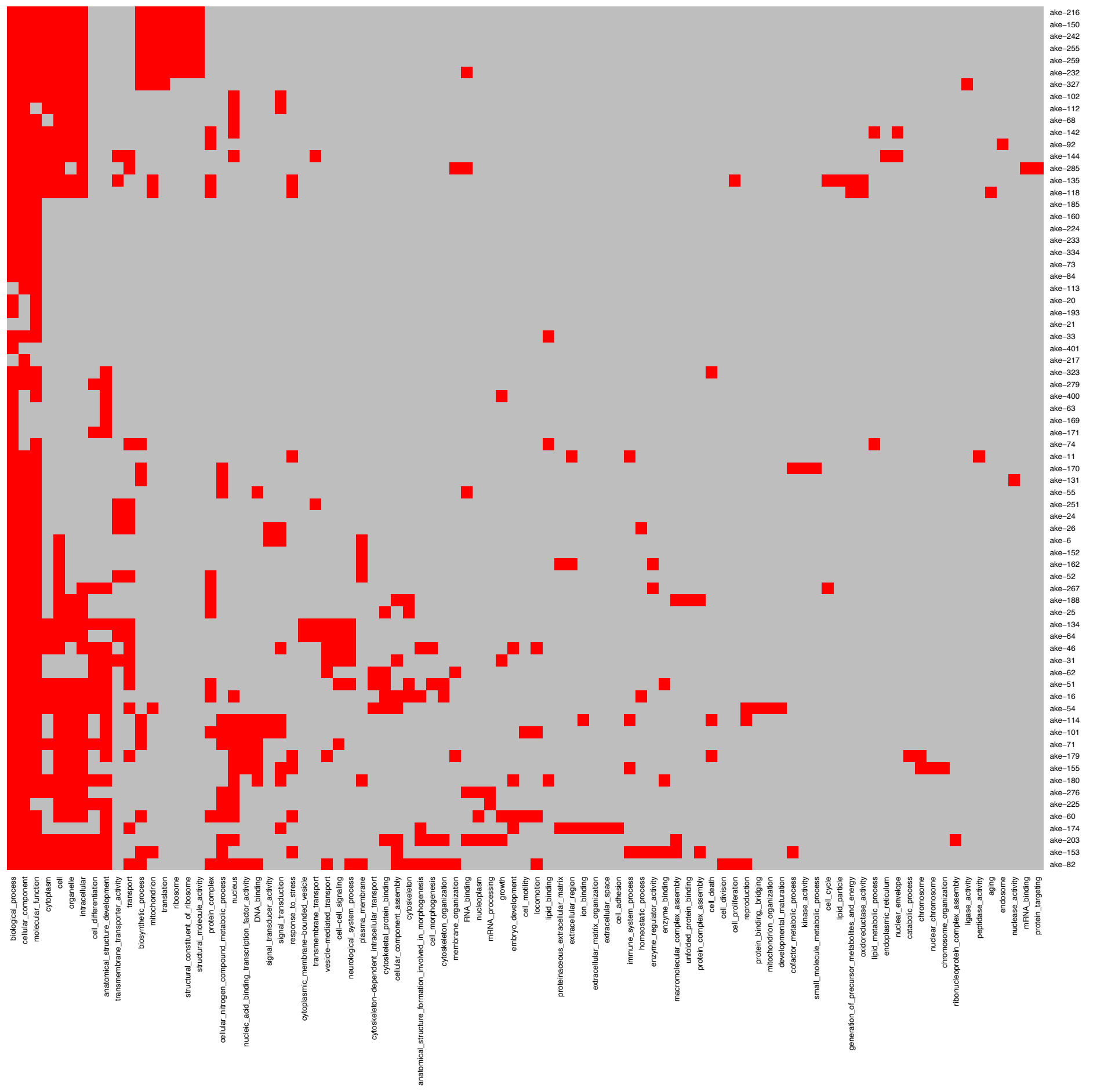

Figure S2. Supplementary Figure related to Figure 4. Absence/presence map of GO-slim terms. $x$-axis: GO-slim terms (including all GO levels); right $y$-axis: internal COP IDs. 
bioRxiv preprint doi: https://doi.org/10.1101/786723; this version posted September 30, 2019. The copyright holder for this preprint (which was not certified by peer review) is the author/funder, who has granted bioRxiv a license to display the preprint in perpetuity. It is made available under aCC-BY-NC-ND 4.0 International license.

D. melanogaster
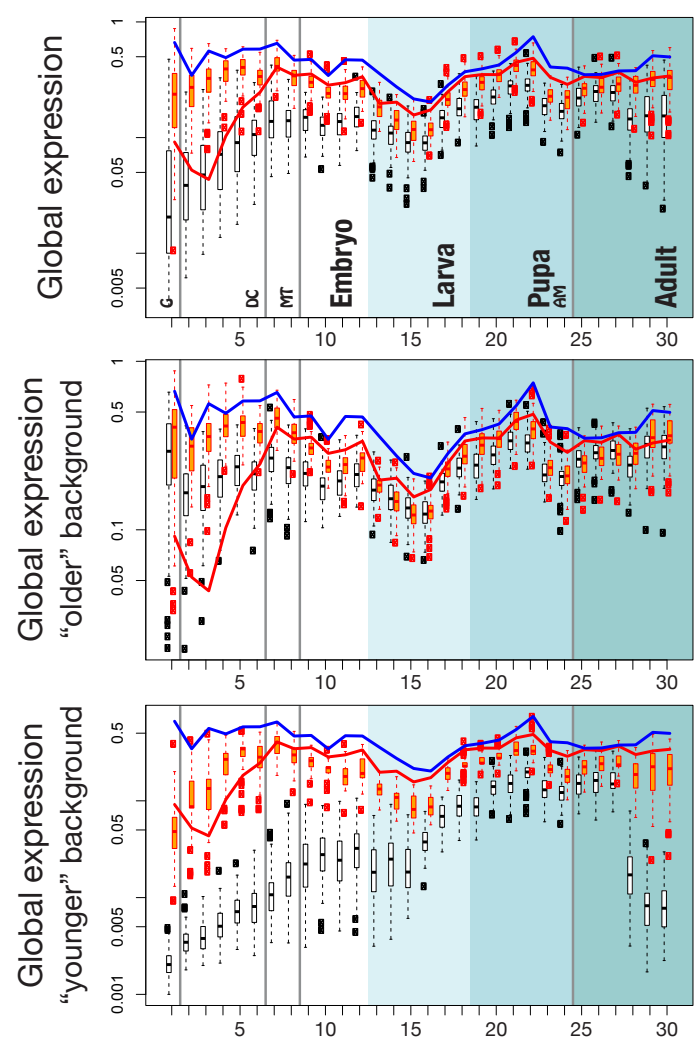

C. elegans
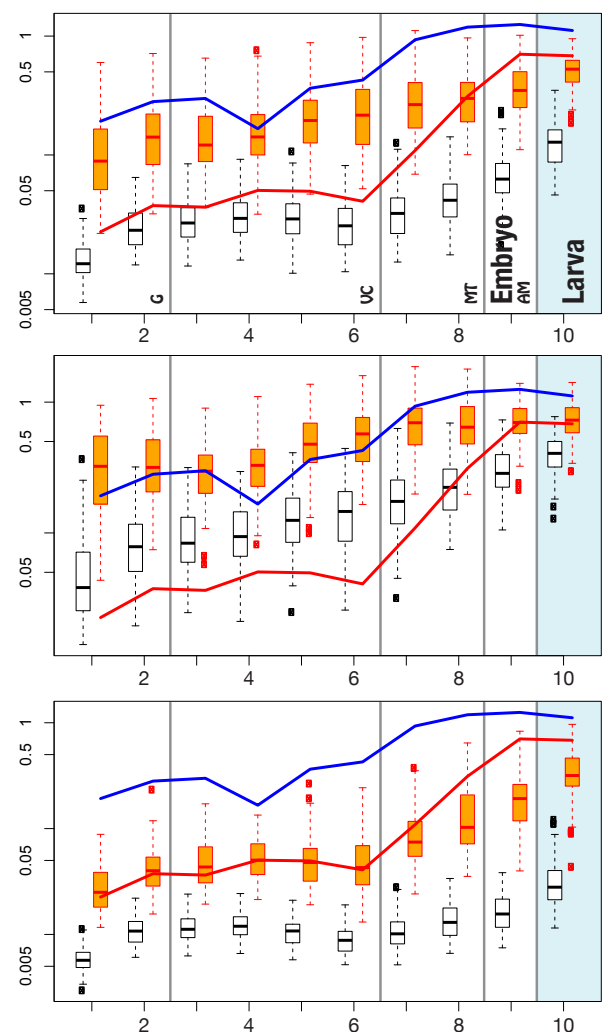

D. rerio
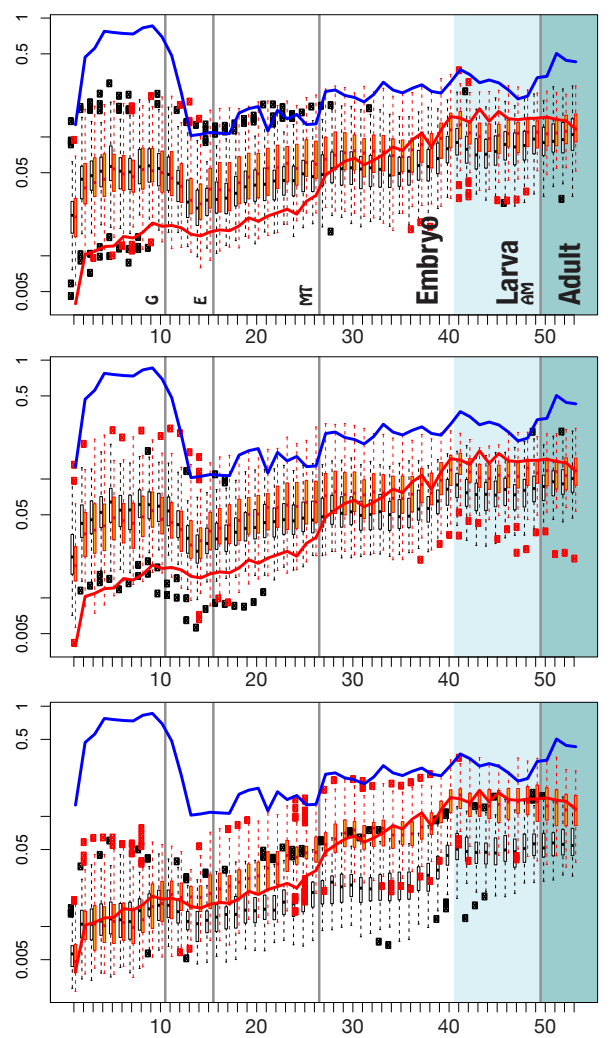

Figure S3. Supplementary Figure related to Figure 5. Expression profiles with background distribution based on medians, instead of means. Color code and other labels as in Fig 2. 


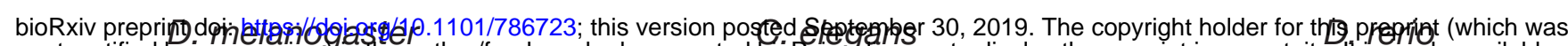
not certified by peer review) is the author/funder, who has granted biöRxiv alicense to display the preprint in perpetuity. It is made available

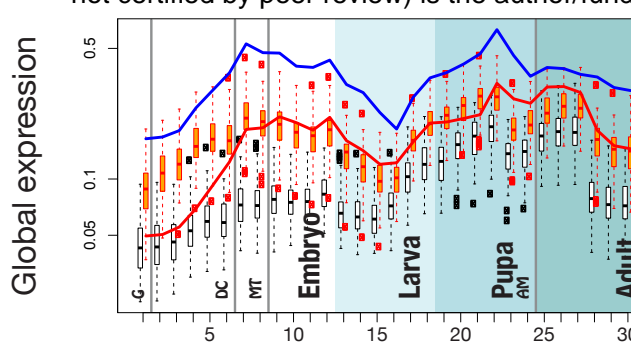

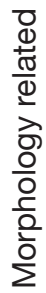
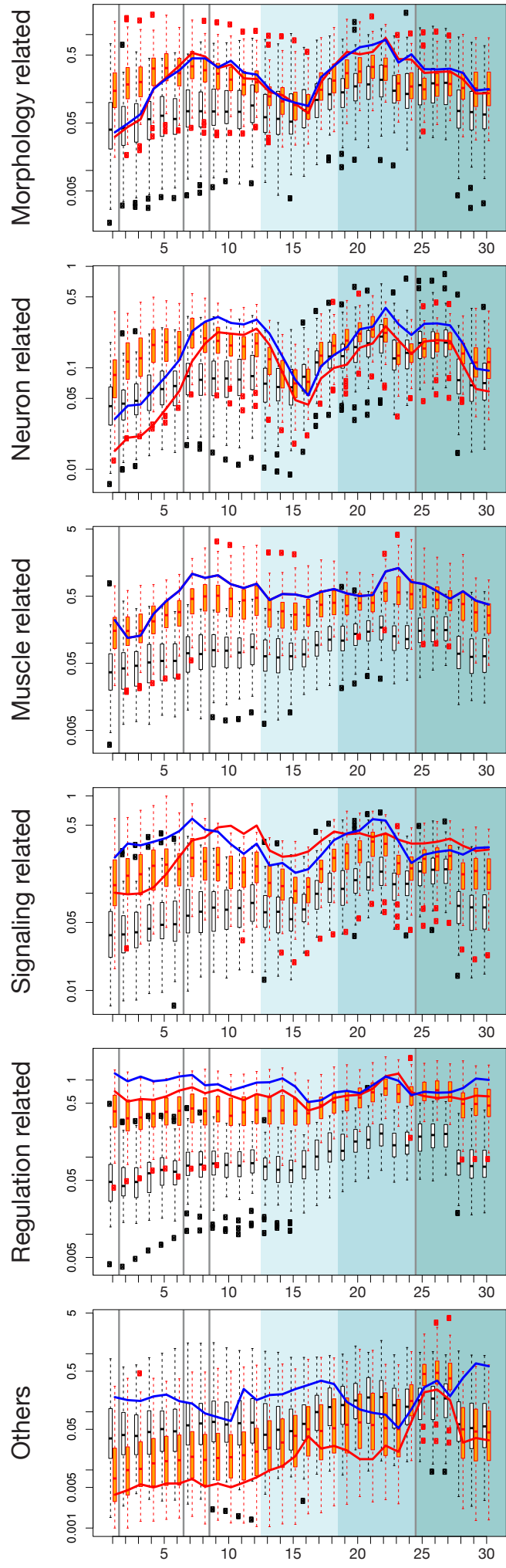
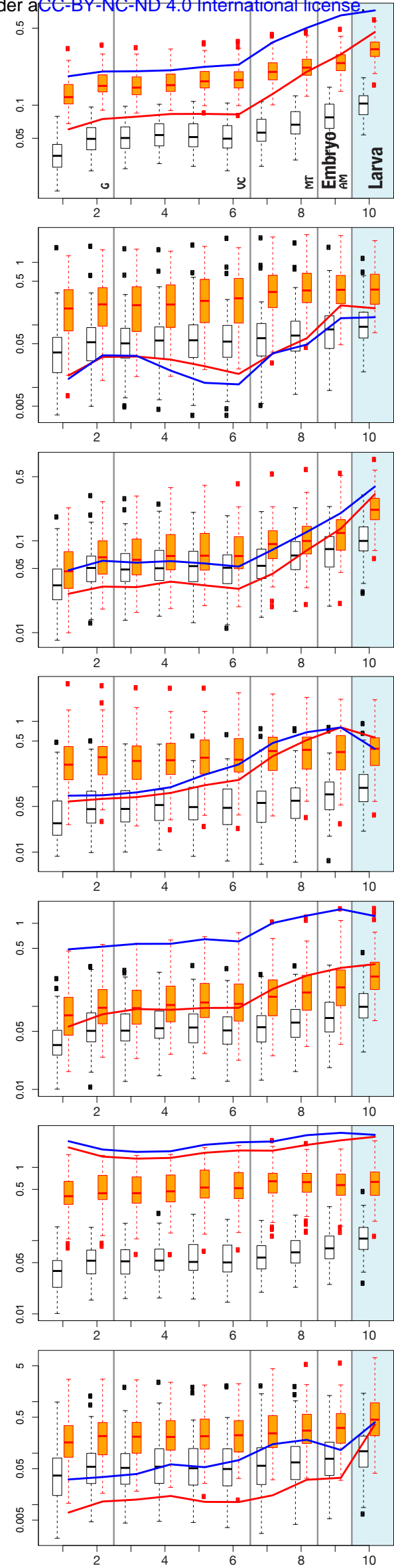
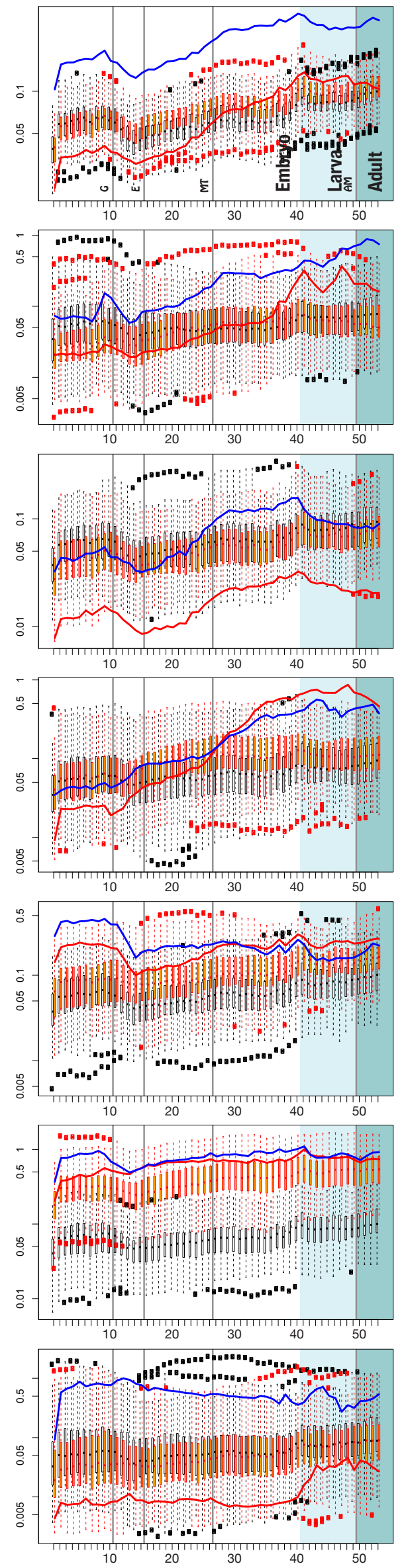

Figure S4. Supplementary Figure related to Figure 5. Expression profiles of set $L^{\prime}$ genes by functional classes. Background distributions based on mean expression values. Color code and other labels as in Fig 2. D.m., 'muscle': there are no paralogs; therefore, only the blue line (for MCOs) is displayed. 
bioRxiv preprint doi: https://doi.org/10.1101/786723; this version posted September 30, 2019. The copyright holder for this preprint (which was not certified by peer review) is the author/funder, who has granted bioRxiv a license to display the preprint in perpetuity. It is made available under aCC-BY-NC-ND 4.0 International license.

Table S1. Supplementary Table related to Figure 1. Distribution of losses in set $L$.

\begin{tabular}{|c|c|c|}
\hline number of clusters & species affected & lineage affected \\
\hline$[33]$ & {$[\mathrm{APL}]$} & [APL only] \\
\hline 31 & $\mathrm{APL}^{*}, \mathrm{CEL}$ & CEL only \\
\hline 22 & $\mathrm{APL}^{*}, \mathrm{STR}$ & STR only \\
\hline 8 & $\mathrm{APL}^{*}, \mathrm{HEL}$ & HEL only \\
\hline 6 & $\mathrm{APL}^{*}, \mathrm{ANO}, \mathrm{DRO}$ & insects \\
\hline 6 & $\mathrm{APL}^{*}, \mathrm{ANO}$ & ANO only \\
\hline 4 & $\mathrm{APL}^{*}, \mathrm{MUS}$ & MUS only \\
\hline 4 & $\mathrm{APL}^{*}, \mathrm{LOT}$ & LOT only \\
\hline 4 & $\mathrm{APL}^{*}, \mathrm{CAP}$ & CAP only \\
\hline 3 & $\mathrm{APL}^{*}, \mathrm{DRO}$ & DRO only \\
\hline 2 & $\mathrm{APL}^{*}, \mathrm{HEL}, \mathrm{CAP}$ & annelids \\
\hline 1 & $\mathrm{ANO}$ & ANO only \\
\hline 1 & - & - \\
\hline \multicolumn{3}{|c|}{$\begin{array}{l}\text { *due to the incomplete assembly and annotation status of the A. califor- } \\
\text { nica genome, APL was ignored when determining the number of losses. } \\
\text { When } A \text {. californica is included only one cluster contains orthologs from } \\
\text { all species (last line). Omitting } A \text {. californica, we find } 33 \text { clusters which } \\
\text { contain orthologs from nine species (first line). In the remaining } 91 \text { clus- } \\
\text { ters in set } L \text { an ortholog from at least one species is missing. Most losses } \\
\text { (31 of } 91) \text { are } C \text {. elegans-specific (second line). }\end{array}$} \\
\hline
\end{tabular}


bioRxiv preprint doi: https://doi.org/10.1101/786723; this version posted September 30, 2019. The copyright holder for this preprint (which was not certified by peer review) is the author/funder, who has granted bioRxiv a license to display the preprint in perpetuity. It is made available under aCC-BY-NC-ND 4.0 International license.

Table S2. Supplementary Table with annotation of $x$-axis tick marks in Fig 2 , Fig S3 and Fig S4 according to [75] (D. melanogaster), [124] (C. elegans) and [48] (D. rerio).

\begin{tabular}{|c|c|c|c|c|c|}
\hline \multirow{2}{*}{$\begin{array}{l}\text { bckgr-color } \\
\text { white }\end{array}$} & \multicolumn{2}{|c|}{$\begin{array}{l}\text { tickmark devel. stage } \\
\text { D. rerio }\end{array}$} & \multicolumn{3}{|c|}{$\begin{array}{cc}\text { bckgr-color } & \text { tickmark devel. stage } \\
& \text { D. melanogaster } \\
\end{array}$} \\
\hline & 1 & egg $0 \mathrm{~min}$ & \multirow[t]{12}{*}{ white } & 1 & embryos $0-2 \mathrm{hr}$ \\
\hline & 2 & zygote $15 \mathrm{~min}$ & & 2 & embryos $2-4 \mathrm{hr}$ \\
\hline & 3 & cleavage $45 \mathrm{~min}$ & & 3 & embryos 4-6hr \\
\hline & 4 & cleavage $1 \mathrm{~h} 15 \mathrm{~min}$ & & 4 & embryos $6-8 \mathrm{hr}$ \\
\hline & 5 & cleavage $1 \mathrm{~h} 45 \mathrm{~min}$ & & 5 & embryos 8-10hr \\
\hline & 6 & blastula $2 \mathrm{~h} 15 \mathrm{~min}$ & & 6 & embryos $10-12 \mathrm{hr}$ \\
\hline & 7 & blastula $2 \mathrm{~h} 45 \mathrm{~min}$ & & 7 & embryos $12-14 \mathrm{hr}$ \\
\hline & 8 & blastula $3 \mathrm{~h} 20 \mathrm{~min}$ & & 8 & embryos $14-16 \mathrm{hr}$ \\
\hline & 9 & blastula $4 \mathrm{~h}$ & & 9 & embryos $16-18 \mathrm{hr}$ \\
\hline & 10 & blastula 4h40min & & 10 & embryos $18-20 \mathrm{hr}$ \\
\hline & 11 & gastrula $5 \mathrm{~h} 20 \mathrm{~min}$ & & 11 & embryos $20-22 \mathrm{hr}$ \\
\hline & 12 & gastrula $6 \mathrm{~h}$ & & 12 & embryos $22-24 \mathrm{hr}$ \\
\hline & 13 & gastrula $7 \mathrm{~h}$ & \multirow[t]{6}{*}{ light grey } & 13 & L1 larvae \\
\hline & 14 & gastrula $8 \mathrm{~h}$ & & 14 & L2 larvae \\
\hline & 15 & gastrula $9 \mathrm{~h}$ & & 15 & L3 larvae $12 \mathrm{hr}$ post molt \\
\hline & 16 & gastrula $10 \mathrm{~h}$ & & 16 & L3 larvaePS 1-2 \\
\hline & 17 & segmentation $10 \mathrm{~h} 20 \mathrm{~min}$ & & 17 & L3 larvaePS 3-6 \\
\hline & 18 & segmentation $11 \mathrm{~h}$ & & 18 & L3 larvaePS 7-9 \\
\hline & 19 & segmentation $11 \mathrm{~h} 40 \mathrm{~min}$ & \multirow[t]{6}{*}{ grey } & 19 & white prepupae \\
\hline & 20 & segmentation $12 \mathrm{~h}$ & & 20 & WPP $12 \mathrm{hr}$ \\
\hline & 21 & segmentation $13 \mathrm{~h}$ & & 21 & WPP 24hr \\
\hline & 22 & segmentation $14 \mathrm{~h}$ & & 22 & pupae WPP 2d \\
\hline & 23 & segmentation $15 \mathrm{~h}$ & & 23 & pupae WPP 3d \\
\hline & 24 & segmentation $16 \mathrm{~h}$ & & 24 & pupae WPP 4d \\
\hline & 25 & segmentation $17 \mathrm{~h}$ & \multirow{11}{*}{ dark grey } & 25 & adult male $1 \mathrm{~d}$ \\
\hline & 26 & segmentation $18 \mathrm{~h}$ & & 26 & adult male $30 \mathrm{~d}$ \\
\hline & 27 & segmentation $19 \mathrm{~h}$ & & 27 & adult male $5 \mathrm{~d}$ \\
\hline & 28 & segmentation $20 \mathrm{~h}$ & & 28 & adult female $1 \mathrm{~d}$ \\
\hline & 29 & segmentation $21 \mathrm{~h}$ & & 29 & adult female $5 \mathrm{~d}$ \\
\hline & 30 & segmentation $22 \mathrm{~h}$ & & 30 & adult female $30 \mathrm{~d}$ \\
\hline & 31 & segmentation $23 \mathrm{~h}$ & & & \\
\hline & 32 & pharyngula $1 \mathrm{~d} 1 \mathrm{~h}$ & & & \\
\hline & 33 & pharyngula $1 \mathrm{~d} 3 \mathrm{~h}$ & & & \\
\hline & 34 & pharyngula $1 \mathrm{~d} 6 \mathrm{~h}$ & & & \\
\hline & 35 & pharyngula $1 \mathrm{~d} 10 \mathrm{~h}$ & & \multicolumn{2}{|c|}{ C. elegans } \\
\hline & 36 & pharyngula $1 \mathrm{~d} 14 \mathrm{~h}$ & & & \\
\hline & 37 & pharyngula $1 \mathrm{~d} 18 \mathrm{~h}$ & white & 1 & stage1 \\
\hline & 38 & hatching $2 \mathrm{~d}$ & & 2 & stage2 \\
\hline & 39 & hatching $2 \mathrm{~d} 12 \mathrm{~h}$ & & 3 & stage3 \\
\hline & 40 & hatching $3 \mathrm{~d}$ & & 4 & stage4 \\
\hline \multirow[t]{9}{*}{ light grey } & 41 & larva $4 \mathrm{~d}$ & & 5 & stage 5 \\
\hline & 42 & larva $6 \mathrm{~d}$ & & 6 & stage6 \\
\hline & 43 & larva $8 \mathrm{~d}$ & & 7 & stage7 \\
\hline & 44 & larva $10 \mathrm{~d}$ & & 8 & stage8 \\
\hline & 45 & larva $14 \mathrm{~d}$ & & 9 & stage9 \\
\hline & 46 & larva $18 \mathrm{~d}$ & light grey & 10 & stage10 \\
\hline & 47 & larva $24 \mathrm{~d}$ & & & \\
\hline & 48 & larva $30 \mathrm{~d}$ & & & \\
\hline & 49 & larva $40 \mathrm{~d}$ & & & \\
\hline \multirow[t]{4}{*}{ dark grey } & 50 & juvenile $45 \mathrm{~d}$ & & & \\
\hline & 51 & juvenile $55 \mathrm{~d}$ & & & \\
\hline & 52 & juvenile $65 \mathrm{~d}$ & & & \\
\hline & 53 & juvenile $80 \mathrm{~d}$ & & & \\
\hline
\end{tabular}


bioRxiv preprint doi: https://doi.org/10.1101/786723; this version posted September 30, 2019. The copyright holder for this preprint (which was not certified by peer review) is the author/funder, who has granted bioRxiv a license to display the preprint in perpetuity. It is made available under aCC-BY-NC-ND 4.0 International license.

Table S3. Suppletary Table related to Fig S3 and Fig S4. Comparison of developmental stages among Drosophila, Caenorhabditis, and Danio

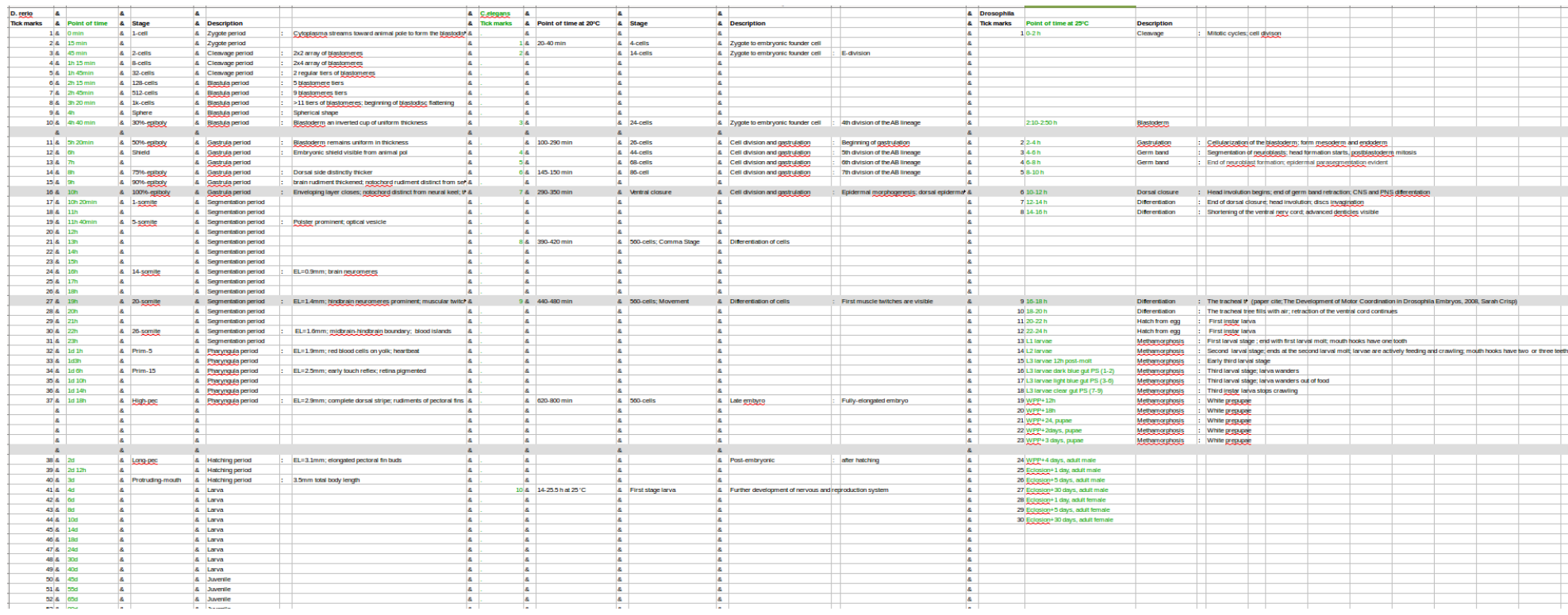


bioRxiv preprint doi: https://doi.org/10.1101/786723; this version posted September 30, 2019. The copyright holder for this preprint (which was not certified by peer review) is the author/funder, who has granted bioRxiv a license to display the preprint in perpetuity. It is made available under aCC-BY-NC-ND 4.0 International license.

Table S4. Supplementary Table related to Figures 3 and 4 . GO terms (of level $\geq 4$ ) identified in 85 COPs of set $L^{\prime}$. COPs ake-68, ake-73, ake-185, ake-217 and ake-224 did not contain GO terms of level $\geq 4$ and do therefore not occur in this table.

\begin{tabular}{|c|c|c|c|}
\hline GO-ID & classification & GO description & $\begin{array}{lll}\text { internal COP-ID (ake- } \\
\# \# \#)\end{array}$ \\
\hline GO:0000122 & regulation & $\begin{array}{l}\text { negative regulation of transcription from RNA } \\
\text { polymerase II promoter }\end{array}$ & $114,37,82$ \\
\hline GO:0000188 & signalling & inactivation of MAPK activity & 3 \\
\hline GO:0000226 & muscle & microtubule cytoskeleton organization & 120,51 \\
\hline GO:0000278 & morphology & mitotic cell cycle & 135,267 \\
\hline GO:0000280 & signalling & nuclear division & 16 \\
\hline GO:0000302 & signalling & response to reactive oxygen species & 118 \\
\hline GO:0000381 & regulation & $\begin{array}{l}\text { regulation of alternative nuclear mRNA splicing, } \\
\text { via spliceosome }\end{array}$ & 276 \\
\hline GO:0000387 & signalling & spliceosomal snRNP assembly & 203 \\
\hline GO:0000422 & regulation & mitochondrion degradation & 193 \\
\hline GO:0001525 & morphology & angiogenesis & 6 \\
\hline GO:0001653 & signalling & peptide receptor activity & 6 \\
\hline GO:0001666 & signalling & response to hypoxia & 135,193 \\
\hline GO:0001708 & morphology & cell fate specification & 82 \\
\hline GO:0001709 & morphology & cell fate determination & 32 \\
\hline GO:0001751 & neuron & compound eye photoreceptor cell differentiation & 180,32 \\
\hline GO:0001754 & neuron & eye photoreceptor cell differentiation & 64,82 \\
\hline GO:0001756 & morphology & somitogenesis & $2,37,6$ \\
\hline GO:0001946 & morphology & lymphangiogenesis & 82 \\
\hline GO:0001947 & muscle & heart looping & 3 \\
\hline GO:0001964 & signalling & startle response & 37,55 \\
\hline GO:0002009 & morphology & morphogenesis of an epithelium & $120,16,171,92$ \\
\hline GO:0002074 & muscle & extraocular skeletal muscle development & 71 \\
\hline GO:0002119 & morphology & nematode larval development & $\begin{array}{l}114,131,150,155,16, \\
171,174,180,18,203, \\
216,232,242,255,259, \\
279,46,71,82,92,97\end{array}$ \\
\hline GO:0002121 & signalling & inter-male aggressive behavior & 52,55 \\
\hline GO:0002385 & signalling & mucosal immune response & 155 \\
\hline GO:0003140 & morphology & $\begin{array}{l}\text { determination of left/right asymmetry in lateral } \\
\text { mesoderm }\end{array}$ & 3 \\
\hline GO:0003146 & muscle & heart jogging & 3 \\
\hline GO:0003707 & signalling & steroid hormone receptor activity & 114 \\
\hline GO:0003810 & signalling & $\begin{array}{l}\text { protein-glutamine gamma-glutamyltransferase ac- } \\
\text { tivity }\end{array}$ & 3 \\
\hline GO:0003917 & regulation & DNA topoisomerase type I activity & 3 \\
\hline GO:0003918 & regulation & DNA topoisomerase (ATP-hydrolyzing) activity & 3 \\
\hline GO:0003924 & signalling & GTPase activity & 3 \\
\hline GO:0003951 & regulation & NAD + kinase activity & 170 \\
\hline GO:0003954 & regulation & NADH dehydrogenase activity & 118 \\
\hline GO:0004091 & signalling & carboxylesterase activity & 51 \\
\hline GO:0004129 & signalling & cytochrome-c oxidase activity & 135 \\
\hline GO:0004190 & signalling & aspartic-type endopeptidase activity & 113 \\
\hline GO:0004221 & signalling & ubiquitin thiolesterase activity & 3 \\
\hline GO:0004252 & signalling & serine-type endopeptidase activity & 11 \\
\hline GO:0004308 & signalling & exo-alpha-sialidase activity & 3 \\
\hline GO:0004386 & signalling & helicase activity & 162 \\
\hline GO:0004518 & signalling & nuclease activity & 131 \\
\hline GO:0004519 & signalling & endonuclease activity & 131 \\
\hline GO:0004521 & signalling & endoribonuclease activity & 131 \\
\hline GO:0004553 & signalling & $\begin{array}{l}\text { hydrolase activity, hydrolyzing O-glycosyl com- } \\
\text { pounds }\end{array}$ & 51 \\
\hline GO:0004556 & signalling & alpha-amylase activity & 27 \\
\hline GO:0004672 & signalling & protein kinase activity & 144 \\
\hline GO:0004674 & signalling & protein serine/threonine kinase activity & 144 \\
\hline GO:0004725 & signalling & protein tyrosine phosphatase activity & 3 \\
\hline GO:0004812 & regulation & aminoacyl-tRNA ligase activity & 160,32 \\
\hline
\end{tabular}


bioRxiv preprint doi: https://doi.org/10.1101/786723; this version posted September 30, 2019. The copyright holder for this preprint (which was not certified by peer review) is the author/funder, who has granted bioRxiv a license to display the preprint in perpetuity. It is made available

... Table S4 continued under aCC-BY-NC-ND 4.0 International license.

\begin{tabular}{|c|c|c|c|}
\hline GO-ID & classification & GO description & $\begin{array}{l}\text { internal COP-ID (ake- } \\
\# \# \#)\end{array}$ \\
\hline GO:0004842 & signalling & ubiquitin-protein ligase activity & 2 \\
\hline GO:0004887 & signalling & thyroid hormone receptor activity & 114 \\
\hline GO:0004888 & signalling & transmembrane signaling receptor activity & 6 \\
\hline GO:0004930 & signalling & G-protein coupled receptor activity & 3,6 \\
\hline GO:0004948 & signalling & calcitonin receptor activity & 6 \\
\hline GO:0004991 & signalling & parathyroid hormone receptor activity & 6 \\
\hline GO:0005085 & signalling & guanyl-nucleotide exchange factor activity & 63 \\
\hline GO:0005086 & signalling & ARF guanyl-nucleotide exchange factor activity & 3 \\
\hline GO:0005089 & signalling & Rho guanyl-nucleotide exchange factor activity & 63 \\
\hline GO:0005184 & neuron & neuropeptide hormone activity & 6 \\
\hline GO:0005216 & signalling & ion channel activity & $144,3,52$ \\
\hline GO:0005253 & signalling & anion channel activity & 19 \\
\hline GO:0005254 & signalling & chloride channel activity & 19,52 \\
\hline GO:0005261 & signalling & cation channel activity & 144 \\
\hline GO:0005262 & signalling & calcium channel activity & 134 \\
\hline GO:0005267 & signalling & potassium channel activity & 144 \\
\hline GO:0005277 & signalling & acetylcholine transmembrane transporter activity & 251 \\
\hline GO:0005278 & signalling & acetylcholine:hydrogen antiporter activity & 251 \\
\hline GO:0005326 & neuron & neurotransmitter transporter activity & 31 \\
\hline GO:0005430 & neuron & $\begin{array}{l}\text { synaptic vesicle amine transmembrane transporter } \\
\text { activity }\end{array}$ & 64 \\
\hline GO:0005786 & signalling & $\begin{array}{l}\text { signal recognition particle, endoplasmic reticulum } \\
\text { targeting }\end{array}$ & 285 \\
\hline GO:0005802 & signalling & trans-Golgi network & 18 \\
\hline GO:0005913 & signalling & cell-cell adherens junction & 180 \\
\hline GO:0005975 & signalling & carbohydrate metabolic process & 27,51 \\
\hline GO:0006030 & morphology & chitin metabolic process & 2 \\
\hline GO:0006120 & regulation & $\begin{array}{l}\text { mitochondrial electron transport, } \mathrm{NADH} \text { to } \\
\text { ubiquinone }\end{array}$ & 118 \\
\hline GO:0006123 & regulation & $\begin{array}{l}\text { mitochondrial electron transport, cytochrome c } \\
\text { to oxygen }\end{array}$ & 135 \\
\hline GO:0006171 & signalling & cAMP biosynthetic process & 6 \\
\hline GO:0006184 & signalling & GTP catabolic process & 3 \\
\hline GO:0006200 & regulation & ATP catabolic process & 162,3 \\
\hline GO:0006260 & regulation & DNA replication & 3 \\
\hline GO:0006265 & regulation & DNA topological change & 3 \\
\hline GO:0006281 & regulation & DNA repair & 33 \\
\hline GO:0006306 & regulation & DNA methylation & 6 \\
\hline GO:0006351 & regulation & transcription, DNA-dependent & $101,2,3,37,71$ \\
\hline GO:0006355 & regulation & regulation of transcription, DNA-dependent & $\begin{array}{l}101,114,2,32,37,3,71 \\
82\end{array}$ \\
\hline GO:0006357 & regulation & $\begin{array}{l}\text { regulation of transcription from RNA polymerase } \\
\text { II promoter }\end{array}$ & 101,37 \\
\hline GO:0006364 & regulation & rRNA processing & 401 \\
\hline GO:0006396 & regulation & RNA processing & 225 \\
\hline GO:0006397 & signalling & mRNA processing & 203,276 \\
\hline GO:0006412 & regulation & translation & $\begin{array}{l}150,216,232,242,255 \\
259,327,97\end{array}$ \\
\hline GO:0006418 & regulation & tRNA aminoacylation for protein translation & 160,32 \\
\hline GO:0006450 & regulation & regulation of translational fidelity & 327 \\
\hline GO:0006457 & signalling & protein folding & 112 \\
\hline GO:0006468 & signalling & protein phosphorylation & 144 \\
\hline GO:0006470 & signalling & protein dephosphorylation & 3 \\
\hline GO:0006486 & signalling & protein glycosylation & 2,3 \\
\hline GO:0006508 & signalling & proteolysis & 113,3 \\
\hline GO:0006511 & signalling & ubiquitin-dependent protein catabolic process & 3 \\
\hline GO:0006614 & regulation & $\begin{array}{l}\text { SRP-dependent cotranslational protein targeting } \\
\text { to membrane }\end{array}$ & 285 \\
\hline GO:0006629 & signalling & lipid metabolic process & 142 \\
\hline GO:0006694 & signalling & steroid biosynthetic process & 74 \\
\hline GO:0006754 & regulation & ATP biosynthetic process & 3 \\
\hline
\end{tabular}


bioRxiv preprint doi: https://doi.org/10.1101/786723; this version posted September 30, 2019. The copyright holder for this preprint (which was not certified by peer review) is the author/funder, who has granted bioRxiv a license to display the preprint in perpetuity. It is made available

... Table S4 continued under aCC-BY-NC-ND 4.0 International license.

\begin{tabular}{|c|c|c|c|}
\hline GO-ID & classification & GO description & $\begin{array}{l}\text { internal COP-ID (ake- } \\
\# \# \#)\end{array}$ \\
\hline GO:0006811 & signalling & ion transport & $144,3,52$ \\
\hline GO:0006812 & signalling & cation transport & 3 \\
\hline GO:0006813 & signalling & potassium ion transport & 144,26 \\
\hline GO:0006814 & signalling & sodium ion transport & 24,3 \\
\hline GO:0006820 & signalling & anion transport & 19 \\
\hline GO:0006821 & signalling & chloride transport & 19,52 \\
\hline GO:0006826 & signalling & iron ion transport & 3 \\
\hline GO:0006836 & neuron & neurotransmitter transport & $101,251,31,64$ \\
\hline GO:0006855 & signalling & drug transmembrane transport & 251,64 \\
\hline GO:0006869 & signalling & lipid transport & 74 \\
\hline GO:0006874 & signalling & cellular calcium ion homeostasis & 5 \\
\hline GO:0006879 & signalling & cellular iron ion homeostasis & 3 \\
\hline GO:0006884 & signalling & cell volume homeostasis & 19 \\
\hline GO:0006885 & signalling & regulation of $\mathrm{pH}$ & 3 \\
\hline GO:0006886 & signalling & intracellular protein transport & 14 \\
\hline GO:0006898 & signalling & receptor-mediated endocytosis & $\begin{array}{l}102,120,131,150,232, \\
242,259,279,46,92\end{array}$ \\
\hline GO:0006911 & signalling & phagocytosis, engulfment & $52,62,82$ \\
\hline GO:0006914 & signalling & autophagy & 193 \\
\hline GO:0006915 & signalling & apoptotic process & 3 \\
\hline GO:0006952 & signalling & defense response & 152,2 \\
\hline GO:0007005 & regulation & mitochondrion organization & 5 \\
\hline GO:0007015 & neuron & actin filament organization & 120 \\
\hline GO:0007021 & muscle & tubulin complex assembly & 188 \\
\hline GO:0007026 & muscle & $\begin{array}{l}\text { negative regulation of microtubule depolymeriza- } \\
\text { tion }\end{array}$ & 25 \\
\hline GO:0007030 & signalling & Golgi organization & 135 \\
\hline GO:0007165 & signalling & signal transduction & $101,112,174,180,2,3,6$ \\
\hline GO:0007166 & signalling & cell surface receptor signaling pathway & 6 \\
\hline GO:0007173 & signalling & $\begin{array}{l}\text { epidermal growth factor receptor signaling path- } \\
\text { way }\end{array}$ & 37 \\
\hline GO:0007186 & signalling & G-protein coupled receptor signaling pathway & 3,6 \\
\hline GO:0007218 & neuron & neuropeptide signaling pathway & 6 \\
\hline GO:0007219 & signalling & Notch signaling pathway & 37,46 \\
\hline GO:0007265 & signalling & Ras protein signal transduction & 180 \\
\hline GO:0007276 & signalling & gamete generation & 171 \\
\hline GO:0007281 & morphology & germ cell development & 276 \\
\hline GO:0007287 & signalling & Nebenkern assembly & 54 \\
\hline GO:0007297 & signalling & ovarian follicle cell migration & 32 \\
\hline GO:0007304 & morphology & chorion-containing eggshell formation & 32 \\
\hline GO:0007349 & signalling & cellularization & 46 \\
\hline GO:0007368 & morphology & determination of left/right symmetry & 3 \\
\hline GO:0007391 & morphology & dorsal closure & 46 \\
\hline GO:0007398 & morphology & ectoderm development & 37 \\
\hline GO:0007399 & neuron & nervous system development & $16,37,46,51,82$ \\
\hline GO:0007400 & neuron & neuroblast fate determination & 82 \\
\hline GO:0007402 & neuron & ganglion mother cell fate determination & 82 \\
\hline GO:0007405 & neuron & neuroblast proliferation & 82 \\
\hline GO:0007406 & neuron & negative regulation of neuroblast proliferation & 82 \\
\hline GO:0007409 & neuron & axonogenesis & $16,203,51,82$ \\
\hline GO:0007411 & neuron & axon guidance & 82 \\
\hline GO:0007416 & neuron & synapse assembly & 82 \\
\hline GO:0007417 & neuron & central nervous system development & 82 \\
\hline GO:0007419 & neuron & ventral cord development & 174,82 \\
\hline GO:0007420 & neuron & brain development & 3,82 \\
\hline GO:0007422 & neuron & peripheral nervous system development & $32,37,82$ \\
\hline GO:0007423 & morphology & sensory organ development & 82 \\
\hline GO:0007424 & morphology & open tracheal system development & 101 \\
\hline GO:0007426 & morphology & tracheal outgrowth, open tracheal system & 180 \\
\hline GO:0007427 & signalling & epithelial cell migration, open tracheal system & 101 \\
\hline GO:0007443 & morphology & Malpighian tubule morphogenesis & 174 \\
\hline
\end{tabular}


bioRxiv preprint doi: https://doi.org/10.1101/786723; this version posted September 30, 2019. The copyright holder for this preprint (which was not certified by peer review) is the author/funder, who has granted bioRxiv a license to display the preprint in perpetuity. It is made available

... Table S4 continued under aCC-BY-NC-ND 4.0 International license.

\begin{tabular}{|c|c|c|c|}
\hline GO-ID & classification & GO description & $\begin{array}{l}\text { internal COP-ID (ake- } \\
\# \# \#)\end{array}$ \\
\hline GO:0007465 & neuron & R7 cell fate commitment & 82 \\
\hline GO:0007476 & morphology & imaginal disc-derived wing morphogenesis & 180 \\
\hline GO:0007498 & morphology & mesoderm development & $174,37,5$ \\
\hline GO:0007507 & muscle & heart development & $16,2,60$ \\
\hline GO:0007517 & muscle & muscle organ development & 16,71 \\
\hline GO:0007519 & muscle & skeletal muscle tissue development & 16,71 \\
\hline GO:0007527 & muscle & adult somatic muscle development & 169 \\
\hline GO:0007528 & neuron & neuromuscular junction development & 203,51 \\
\hline GO:0007552 & morphology & metamorphosis & 114 \\
\hline GO:0007553 & morphology & regulation of ecdysteroid metabolic process & 114 \\
\hline GO:0007591 & morphology & molting cycle, chitin-based cuticle & 114 \\
\hline GO:0007606 & signalling & sensory perception of chemical stimulus & 3 \\
\hline GO:0007616 & neuron & long-term memory & 22 \\
\hline GO:0007619 & signalling & courtship behavior & 82 \\
\hline GO:0008026 & regulation & ATP-dependent helicase activity & 162 \\
\hline GO:0008036 & signalling & diuretic hormone receptor activity & 6 \\
\hline GO:0008045 & neuron & motor axon guidance & 46 \\
\hline GO:0008049 & signalling & male courtship behavior & 6 \\
\hline GO:0008081 & signalling & phosphoric diester hydrolase activity & 33 \\
\hline GO:0008083 & signalling & growth factor activity & 3 \\
\hline GO:0008088 & neuron & axon cargo transport & 51,62 \\
\hline GO:0008101 & signalling & decapentaplegic signaling pathway & 32 \\
\hline GO:0008104 & signalling & protein localization & 82 \\
\hline GO:0008137 & regulation & NADH dehydrogenase (ubiquinone) activity & 118 \\
\hline GO:0008138 & signalling & $\begin{array}{l}\text { protein tyrosine/serine/threonine phosphatase ac- } \\
\text { tivity }\end{array}$ & 3 \\
\hline GO:0008168 & signalling & methyltransferase activity & 6 \\
\hline GO:0008188 & neuron & neuropeptide receptor activity & 6 \\
\hline GO:0008233 & signalling & peptidase activity & 3 \\
\hline GO:0008234 & signalling & cysteine-type peptidase activity & 3 \\
\hline GO:0008284 & signalling & positive regulation of cell proliferation & 32 \\
\hline GO:0008285 & signalling & negative regulation of cell proliferation & 82 \\
\hline GO:0008293 & signalling & torso signaling pathway & 180 \\
\hline GO:0008355 & neuron & olfactory learning & 51 \\
\hline GO:0008356 & morphology & asymmetric cell division & 82 \\
\hline GO:0008373 & signalling & sialyltransferase activity & 3 \\
\hline GO:0008380 & regulation & RNA splicing & 203,276 \\
\hline GO:0008417 & signalling & fucosyltransferase activity & 2 \\
\hline GO:0008504 & signalling & monoamine transmembrane transporter activity & 64 \\
\hline GO:0008508 & signalling & bile acid:sodium symporter activity & 24 \\
\hline GO:0008582 & neuron & $\begin{array}{l}\text { regulation of synaptic growth at neuromuscular } \\
\text { junction }\end{array}$ & 51 \\
\hline GO:0008587 & morphology & imaginal disc-derived wing margin morphogenesis & 37 \\
\hline GO:0008595 & morphology & anterior/posterior axis specification, embryo & 180 \\
\hline GO:0008898 & signalling & homocysteine S-methyltransferase activity & 2 \\
\hline GO:0009303 & regulation & rRNA transcription & 131 \\
\hline GO:0009306 & signalling & protein secretion & 46 \\
\hline GO:0009651 & signalling & response to salt stress & 155 \\
\hline GO:0009792 & morphology & $\begin{array}{l}\text { embryo development ending in birth or egg hatch- } \\
\text { ing }\end{array}$ & $\begin{array}{l}118,131,150,155,16, \\
169,174,193,203,21, \\
216,232,242,255,259, \\
276,323,334,46,51,71, \\
92,97\end{array}$ \\
\hline GO:0009881 & signalling & photoreceptor activity & 26 \\
\hline GO:0009966 & signalling & regulation of signal transduction & 180 \\
\hline GO:0009996 & morphology & negative regulation of cell fate specification & 32 \\
\hline GO:0010001 & neuron & glial cell differentiation & 82 \\
\hline GO:0010171 & morphology & body morphogenesis & $114,120,16,18,71$ \\
\hline GO:0010466 & signalling & negative regulation of peptidase activity & 162 \\
\hline GO:0010468 & signalling & regulation of gene expression & 401 \\
\hline GO:0010629 & signalling & negative regulation of gene expression & 112,37 \\
\hline
\end{tabular}


bioRxiv preprint doi: https://doi.org/10.1101/786723; this version posted September 30, 2019. The copyright holder for this preprint (which was not certified by peer review) is the author/funder, who has granted bioRxiv a license to display the preprint in perpetuity. It is made available

... Table S4 continued under aCC-BY-NC-ND 4.0 International license.

\begin{tabular}{|c|c|c|c|}
\hline GO-ID & classification & GO description & $\begin{array}{l}\text { internal COP-ID (ake- } \\
\# \# \#)\end{array}$ \\
\hline GO:0010866 & signalling & regulation of triglyceride biosynthetic process & 155 \\
\hline GO:0010867 & signalling & $\begin{array}{l}\text { positive regulation of triglyceride biosynthetic pro- } \\
\text { cess }\end{array}$ & 142 \\
\hline GO:0014036 & neuron & neural crest cell fate specification & 3 \\
\hline GO:0014812 & muscle & muscle cell migration & 60 \\
\hline GO:0015077 & signalling & $\begin{array}{l}\text { monovalent inorganic cation transmembrane trans- } \\
\text { porter activity }\end{array}$ & 3 \\
\hline GO:0015143 & signalling & urate transmembrane transporter activity & 21 \\
\hline GO:0015238 & signalling & drug transmembrane transporter activity & 251,64 \\
\hline GO:0015269 & signalling & calcium-activated potassium channel activity & 26 \\
\hline GO:0015297 & signalling & antiporter activity & 3 \\
\hline GO:0015299 & signalling & solute:hydrogen antiporter activity & 3 \\
\hline GO:0015385 & signalling & sodium:hydrogen antiporter activity & 3 \\
\hline GO:0015662 & regulation & $\begin{array}{l}\text { ATPase activity, coupled to transmembrane move- } \\
\text { ment of ions, phosphorylative mechanism }\end{array}$ & 3 \\
\hline GO:0015672 & signalling & monovalent inorganic cation transport & 144,3 \\
\hline GO:0015721 & signalling & bile acid and bile salt transport & 24 \\
\hline GO:0015842 & neuron & synaptic vesicle amine transport & 64 \\
\hline GO:0015844 & signalling & monoamine transport & 64 \\
\hline GO:0015872 & signalling & dopamine transport & 64 \\
\hline GO:0015893 & signalling & drug transport & 251,64 \\
\hline GO:0015934 & signalling & large ribosomal subunit & 232 \\
\hline GO:0015992 & signalling & proton transport & 135,3 \\
\hline GO:0016057 & neuron & $\begin{array}{l}\text { regulation of membrane potential in photoreceptor } \\
\text { cell }\end{array}$ & 26 \\
\hline GO:0016070 & regulation & RNA metabolic process & 55 \\
\hline GO:0016079 & neuron & synaptic vesicle exocytosis & 31 \\
\hline GO:0016202 & muscle & regulation of striated muscle tissue development & 71 \\
\hline GO:0016286 & signalling & $\begin{array}{l}\text { small conductance calcium-activated potassium } \\
\text { channel activity }\end{array}$ & 26 \\
\hline GO:0016301 & signalling & kinase activity & 144,26 \\
\hline GO:0016307 & signalling & phosphatidylinositol phosphate kinase activity & 14,2 \\
\hline GO:0016310 & signalling & phosphorylation & $144,170,26$ \\
\hline GO:0016311 & signalling & dephosphorylation & 3 \\
\hline GO:0016319 & neuron & mushroom body development & 114,52 \\
\hline GO:0016337 & signalling & cell-cell adhesion & 3 \\
\hline GO:0016360 & morphology & sensory organ precursor cell fate determination & 37 \\
\hline GO:0016477 & morphology & cell migration & 46 \\
\hline GO:0016567 & signalling & protein ubiquitination & 2 \\
\hline GO:0016568 & morphology & chromatin modification & 3 \\
\hline GO:0016746 & signalling & transferase activity, transferring acyl groups & 251 \\
\hline GO:0016757 & signalling & transferase activity, transferring glycosyl groups & 2,3 \\
\hline GO:0016772 & signalling & $\begin{array}{l}\text { transferase activity, transferring phosphorus- } \\
\text { containing groups }\end{array}$ & 144 \\
\hline GO:0016791 & signalling & phosphatase activity & 3 \\
\hline GO:0016798 & signalling & hydrolase activity, acting on glycosyl bonds & 27 \\
\hline GO:0016820 & signalling & $\begin{array}{l}\text { hydrolase activity, acting on acid anhydrides, cat- } \\
\text { alyzing transmembrane movement of substances }\end{array}$ & 3 \\
\hline GO:0016887 & regulation & ATPase activity & 3 \\
\hline GO:0017017 & signalling & $\begin{array}{l}\text { MAP kinase tyrosine/serine/threonine phos- } \\
\text { phatase activity }\end{array}$ & 3 \\
\hline GO:0017053 & signalling & transcriptional repressor complex & 37 \\
\hline GO:0017127 & signalling & cholesterol transporter activity & 74 \\
\hline GO:0018149 & signalling & peptide cross-linking & 3 \\
\hline GO:0018990 & morphology & ecdysis, chitin-based cuticle & 114 \\
\hline GO:0018996 & morphology & molting cycle, collagen and cuticulin-based cuticle & $114,131,46,82,92$ \\
\hline GO:0019730 & signalling & antimicrobial humoral response & 114 \\
\hline GO:0019888 & signalling & protein phosphatase regulator activity & 267 \\
\hline GO:0019895 & regulation & kinesin-associated mitochondrial adaptor activity & 54 \\
\hline GO:0019896 & neuron & axon transport of mitochondrion & 54 \\
\hline GO:0019915 & signalling & lipid storage & $179,19,20,22,51,5,74$ \\
\hline
\end{tabular}


bioRxiv preprint doi: https://doi.org/10.1101/786723; this version posted September 30, 2019. The copyright holder for this preprint (which was not certified by peer review) is the author/funder, who has granted bioRxiv a license to display the preprint in perpetuity. It is made available

... Table S4 continued under aCC-BY-NC-ND 4.0 International license.

\begin{tabular}{|c|c|c|c|}
\hline GO-ID & classification & GO description & $\begin{array}{l}\text { internal COP-ID (ake- } \\
\# \# \#)\end{array}$ \\
\hline GO:0021884 & neuron & forebrain neuron development & 82 \\
\hline GO:0021905 & neuron & forebrain-midbrain boundary formation & 3 \\
\hline GO:0022008 & neuron & neurogenesis & $171,267,279,46,62$ \\
\hline GO:0030001 & signalling & metal ion transport & 18 \\
\hline GO:0030097 & signalling & hemopoiesis & 18 \\
\hline GO:0030111 & signalling & regulation of Wnt receptor signaling pathway & 400,60 \\
\hline GO:0030154 & morphology & cell differentiation & 71 \\
\hline GO:0030163 & signalling & protein catabolic process & 92 \\
\hline GO:0030239 & muscle & myofibril assembly & $16,5,82$ \\
\hline GO:0030240 & muscle & skeletal muscle thin filament assembly & 203,5 \\
\hline GO:0030289 & signalling & protein phosphatase 4 complex & 267 \\
\hline GO:0030301 & signalling & cholesterol transport & 74 \\
\hline GO:0030307 & signalling & positive regulation of cell growth & 32 \\
\hline GO:0030382 & regulation & sperm mitochondrion organization & 54 \\
\hline GO:0030414 & signalling & peptidase inhibitor activity & 162 \\
\hline GO:0030421 & signalling & defecation & 16 \\
\hline GO:0030707 & signalling & ovarian follicle cell development & 32 \\
\hline GO:0030916 & signalling & otic vesicle formation & 174 \\
\hline GO:0031016 & morphology & pancreas development & 60 \\
\hline GO:0031453 & signalling & positive regulation of heterochromatin assembly & 155 \\
\hline GO:0031618 & signalling & nuclear centromeric heterochromatin & 155 \\
\hline GO:0032012 & signalling & regulation of $\mathrm{ARF}$ protein signal transduction & 3 \\
\hline GO:0032474 & morphology & otolith morphogenesis & 174 \\
\hline GO:0034220 & signalling & ion transmembrane transport & $135,3,52$ \\
\hline GO:0034605 & signalling & cellular response to heat & 155 \\
\hline GO:0034707 & signalling & chloride channel complex & 52 \\
\hline GO:0035023 & signalling & regulation of Rho protein signal transduction & 63 \\
\hline GO:0035050 & morphology & embryonic heart tube development & 3 \\
\hline GO:0035071 & signalling & salivary gland cell autophagic cell death & 323,32 \\
\hline GO:0035072 & signalling & $\begin{array}{l}\text { ecdysone-mediated induction of salivary gland cell } \\
\text { autophagic cell death }\end{array}$ & 114 \\
\hline GO:0035098 & signalling & $\mathrm{ESC} / \mathrm{E}(\mathrm{Z})$ complex & 3 \\
\hline GO:0035147 & signalling & branch fusion, open tracheal system & 101 \\
\hline GO:0035188 & morphology & hatching & 267 \\
\hline GO:0035199 & signalling & salt aversion & 3 \\
\hline GO:0035238 & signalling & vitamin A biosynthetic process & 64 \\
\hline GO:0035282 & morphology & segmentation & 32 \\
\hline GO:0035304 & signalling & regulation of protein dephosphorylation & 267 \\
\hline GO:0035307 & signalling & positive regulation of protein dephosphorylation & 142 \\
\hline GO:0035335 & signalling & peptidyl-tyrosine dephosphorylation & 3 \\
\hline GO:0035462 & morphology & $\begin{array}{l}\text { determination of left/right asymmetry in dien- } \\
\text { cephalon }\end{array}$ & 3 \\
\hline GO:0035469 & morphology & determination of pancreatic left/right asymmetry & 3 \\
\hline GO:0035556 & signalling & intracellular signal transduction & 184,63 \\
\hline GO:0036065 & signalling & fucosylation & 2 \\
\hline GO:0040002 & morphology & collagen and cuticulin-based cuticle development & 18 \\
\hline GO:0040010 & signalling & positive regulation of growth rate & $\begin{array}{l}102,131,150,16,171, \\
174,203,216,232,242, \\
255,259,2,279,46,71, \\
82,84,92,97\end{array}$ \\
\hline GO:0040017 & signalling & positive regulation of locomotion & 46 \\
\hline GO:0040018 & morphology & $\begin{array}{l}\text { positive regulation of multicellular organism } \\
\text { growth }\end{array}$ & $114,174,19$ \\
\hline GO:0040020 & morphology & regulation of meiosis & 276 \\
\hline GO:0040026 & morphology & positive regulation of vulval development & 180 \\
\hline GO:0040034 & morphology & regulation of development, heterochronic & 114 \\
\hline GO:0040035 & morphology & hermaphrodite genitalia development & $120,16,171,92$ \\
\hline GO:0042048 & neuron & olfactory behavior & 55 \\
\hline GO:0042051 & neuron & compound eye photoreceptor development & 46 \\
\hline GO:0042063 & neuron & gliogenesis & 37 \\
\hline GO:0042074 & morphology & cell migration involved in gastrulation & 60 \\
\hline
\end{tabular}


bioRxiv preprint doi: https://doi.org/10.1101/786723; this version posted September 30, 2019. The copyright holder for this preprint (which was not certified by peer review) is the author/funder, who has granted bioRxiv a license to display the preprint in perpetuity. It is made available

... Table S4 continued under aCC-BY-NC-ND 4.0 International license.

\begin{tabular}{|c|c|c|c|}
\hline GO-ID & classification & GO description & $\begin{array}{l}\text { internal COP-ID } \\
\# \# \#)\end{array}$ \\
\hline GO:0042127 & morphology & regulation of cell proliferation & 276 \\
\hline GO:0042332 & signalling & gravitaxis & 6 \\
\hline GO:0042673 & neuron & regulation of retinal cone cell fate specification & 82 \\
\hline GO:0042676 & neuron & compound eye cone cell fate commitment & 82 \\
\hline GO:0042745 & morphology & circadian sleep/wake cycle & 6 \\
\hline GO:0042749 & morphology & regulation of circadian sleep/wake cycle & 6 \\
\hline GO:0042803 & signalling & protein homodimerization activity & $155,32,37$ \\
\hline GO:0042981 & signalling & regulation of apoptotic process & 134 \\
\hline GO:0043005 & neuron & neuron projection & 6 \\
\hline GO:0043053 & signalling & dauer entry & 113 \\
\hline GO:0043066 & signalling & negative regulation of apoptotic process & 32 \\
\hline GO:0043086 & signalling & negative regulation of catalytic activity & 153 \\
\hline GO:0043152 & signalling & induction of bacterial agglutination & 2 \\
\hline GO:0043282 & muscle & pharyngeal muscle development & 71 \\
\hline GO:0043401 & signalling & steroid hormone mediated signaling pathway & 114 \\
\hline GO:0043524 & neuron & negative regulation of neuron apoptotic process & 51 \\
\hline GO:0043620 & regulation & $\begin{array}{l}\text { regulation of DNA-dependent transcription in re- } \\
\text { sponse to stress }\end{array}$ & 60 \\
\hline GO:0045087 & signalling & innate immune response & 233 \\
\hline GO:0045165 & morphology & cell fate commitment & 37 \\
\hline GO:0045179 & signalling & apical cortex & 82 \\
\hline GO:0045214 & signalling & sarcomere organization & 16,5 \\
\hline GO:0045468 & neuron & regulation of $R 8$ cell spacing in compound eye & 37 \\
\hline GO:0045475 & neuron & locomotor rhythm & 6 \\
\hline GO:0045500 & neuron & sevenless signaling pathway & 46 \\
\hline GO:0045572 & signalling & positive regulation of imaginal disc growth & 102 \\
\hline GO:0045664 & neuron & regulation of neuron differentiation & 82 \\
\hline GO:0045676 & neuron & regulation of $\mathrm{R} 7$ cell differentiation & 82 \\
\hline GO:0045746 & signalling & negative regulation of Notch signaling pathway & 32 \\
\hline GO:0045793 & signalling & positive regulation of cell size & 120 \\
\hline GO:0045892 & regulation & $\begin{array}{l}\text { negative regulation of transcription, DNA- } \\
\text { dependent }\end{array}$ & 37 \\
\hline GO:0045893 & regulation & $\begin{array}{l}\text { positive regulation of transcription, DNA- } \\
\text { dependent }\end{array}$ & 102 \\
\hline GO:0045900 & regulation & negative regulation of translational elongation & 285 \\
\hline GO:0045944 & signalling & $\begin{array}{l}\text { positive regulation of transcription from RNA } \\
\text { polymerase II promoter }\end{array}$ & $101,114,155$ \\
\hline GO:0045980 & signalling & $\begin{array}{l}\text { negative regulation of nucleotide metabolic pro- } \\
\text { cess }\end{array}$ & 153 \\
\hline GO:0046008 & signalling & regulation of female receptivity, post-mating & 2 \\
\hline GO:0046331 & signalling & lateral inhibition & 18 \\
\hline GO:0046488 & signalling & phosphatidylinositol metabolic process & 14,2 \\
\hline GO:0046692 & signalling & sperm competition & 2 \\
\hline GO:0046716 & muscle & muscle cell homeostasis & 16,5 \\
\hline GO:0046843 & morphology & dorsal appendage formation & 32 \\
\hline GO:0046854 & signalling & phosphatidylinositol phosphorylation & 14,2 \\
\hline GO:0046928 & neuron & regulation of neurotransmitter secretion & 31 \\
\hline GO:0046982 & signalling & protein heterodimerization activity & 101,37 \\
\hline GO:0046983 & signalling & protein dimerization activity & 2 \\
\hline GO:0047291 & signalling & $\begin{array}{l}\text { lactosylceramide alpha-2, 3-sialyltransferase activ- } \\
\text { ity }\end{array}$ & 3 \\
\hline GO:0048102 & signalling & autophagic cell death & 323,32 \\
\hline GO:0048190 & morphology & wing disc dorsal/ventral pattern formation & 323,37 \\
\hline GO:0048311 & regulation & mitochondrion distribution & 54 \\
\hline GO:0048368 & morphology & lateral mesoderm development & 3 \\
\hline GO:0048477 & morphology & oogenesis & 114,32 \\
\hline GO:0048488 & neuron & synaptic vesicle endocytosis & 134,46 \\
\hline GO:0048500 & signalling & signal recognition particle & 285 \\
\hline GO:0048512 & morphology & circadian behavior & 6 \\
\hline GO:0048514 & morphology & blood vessel morphogenesis & 63 \\
\hline GO:0048644 & muscle & muscle organ morphogenesis & 5 \\
\hline
\end{tabular}


bioRxiv preprint doi: https://doi.org/10.1101/786723; this version posted September 30, 2019. The copyright holder for this preprint (which was not certified by peer review) is the author/funder, who has granted bioRxiv a license to display the preprint in perpetuity. It is made available

... Table S4 continued under aCC-BY-NC-ND 4.0 International license.

\begin{tabular}{|c|c|c|c|}
\hline GO-ID & classification & GO description & $\begin{array}{l}\text { internal COP-ID (ake- } \\
\# \# \#)\end{array}$ \\
\hline GO:0048666 & neuron & neuron development & 37 \\
\hline GO:0048676 & neuron & axon extension involved in development & 203 \\
\hline GO:0048738 & muscle & cardiac muscle tissue development & 16 \\
\hline GO:0048741 & muscle & skeletal muscle fiber development & 71 \\
\hline GO:0048749 & neuron & compound eye development & $32,37,51$ \\
\hline GO:0048752 & morphology & semicircular canal morphogenesis & 174 \\
\hline GO:0048755 & neuron & branching morphogenesis of a nerve & 3 \\
\hline GO:0048812 & neuron & neuron projection morphogenesis & 3 \\
\hline GO:0048813 & neuron & dendrite morphogenesis & $37,51,82$ \\
\hline GO:0048844 & morphology & artery morphogenesis & 6 \\
\hline GO:0048846 & neuron & axon extension involved in axon guidance & 14 \\
\hline GO:0048854 & neuron & brain morphogenesis & 37,55 \\
\hline GO:0048885 & neuron & neuromast deposition & 82 \\
\hline GO:0048886 & neuron & neuromast hair cell differentiation & 82 \\
\hline GO:0048920 & neuron & $\begin{array}{l}\text { posterior lateral line neuromast primordium mi- } \\
\text { gration }\end{array}$ & 162 \\
\hline GO:0050768 & neuron & negative regulation of neurogenesis & 37 \\
\hline GO:0050771 & signalling & negative regulation of axonogenesis & 82 \\
\hline GO:0050790 & signalling & regulation of catalytic activity & 3,63 \\
\hline GO:0050830 & signalling & defense response to Gram-positive bacterium & 153 \\
\hline GO:0050909 & signalling & sensory perception of taste & 82 \\
\hline GO:0050914 & signalling & sensory perception of salty taste & 3 \\
\hline GO:0051124 & neuron & synaptic growth at neuromuscular junction & 31 \\
\hline GO:0051216 & morphology & cartilage development & 174,3 \\
\hline GO:0051260 & signalling & protein homooligomerization & 3 \\
\hline GO:0051403 & signalling & stress-activated MAPK cascade & 155 \\
\hline GO:0051608 & signalling & histamine transport & 64 \\
\hline GO:0055002 & muscle & striated muscle cell development & 71 \\
\hline GO:0055059 & neuron & asymmetric neuroblast division & 82 \\
\hline GO:0055060 & neuron & $\begin{array}{l}\text { asymmetric neuroblast division resulting in gan- } \\
\text { glion mother cell formation }\end{array}$ & 82 \\
\hline GO:0060028 & neuron & convergent extension involved in axis elongation & 400,60 \\
\hline GO:0060029 & morphology & convergent extension involved in organogenesis & 60 \\
\hline GO:0060037 & morphology & pharyngeal system development & 174 \\
\hline GO:0060041 & neuron & retina development in camera-type eye & 64 \\
\hline GO:0060052 & neuron & neurofilament cytoskeleton organization & 51 \\
\hline GO:0060385 & neuron & axonogenesis involved in innervation & 82 \\
\hline GO:0060438 & morphology & trachea development & 174 \\
\hline GO:0060857 & neuron & establishment of glial blood-brain barrier & 152 \\
\hline GO:0060971 & morphology & embryonic heart tube left/right pattern formation & 3 \\
\hline GO:0061327 & morphology & anterior Malpighian tubule development & 174 \\
\hline GO:0070278 & signalling & extracellular matrix constituent secretion & 174 \\
\hline GO:0070527 & signalling & platelet aggregation & 3 \\
\hline GO:0070829 & signalling & heterochromatin maintenance & 155 \\
\hline GO:0070983 & neuron & dendrite guidance & 82 \\
\hline GO:0071436 & signalling & sodium ion export & 24,3 \\
\hline GO:0071470 & signalling & cellular response to osmotic stress & 155 \\
\hline GO:0071688 & muscle & striated muscle myosin thick filament assembly & 203,5 \\
\hline GO:0071805 & signalling & potassium ion transmembrane transport & 144,26 \\
\hline GO:0072320 & signalling & volume-sensitive chloride channel activity & 19 \\
\hline GO:0072583 & signalling & clathrin-mediated endocytosis & 46 \\
\hline GO:0090305 & signalling & nucleic acid phosphodiester bond hydrolysis & 131 \\
\hline GO:2000223 & signalling & $\begin{array}{l}\text { regulation of BMP signaling pathway involved in } \\
\text { heart jogging }\end{array}$ & 3 \\
\hline
\end{tabular}


Table S5. Supplementary Table related to Figures 1 and 4 . Description of the 85 clusters in set $L^{\prime}$.

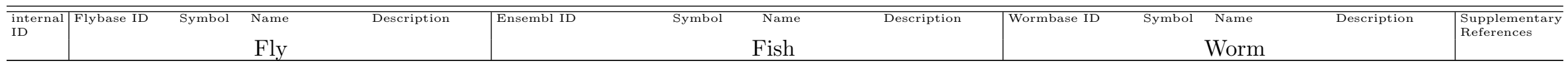

\section{Morphology related}

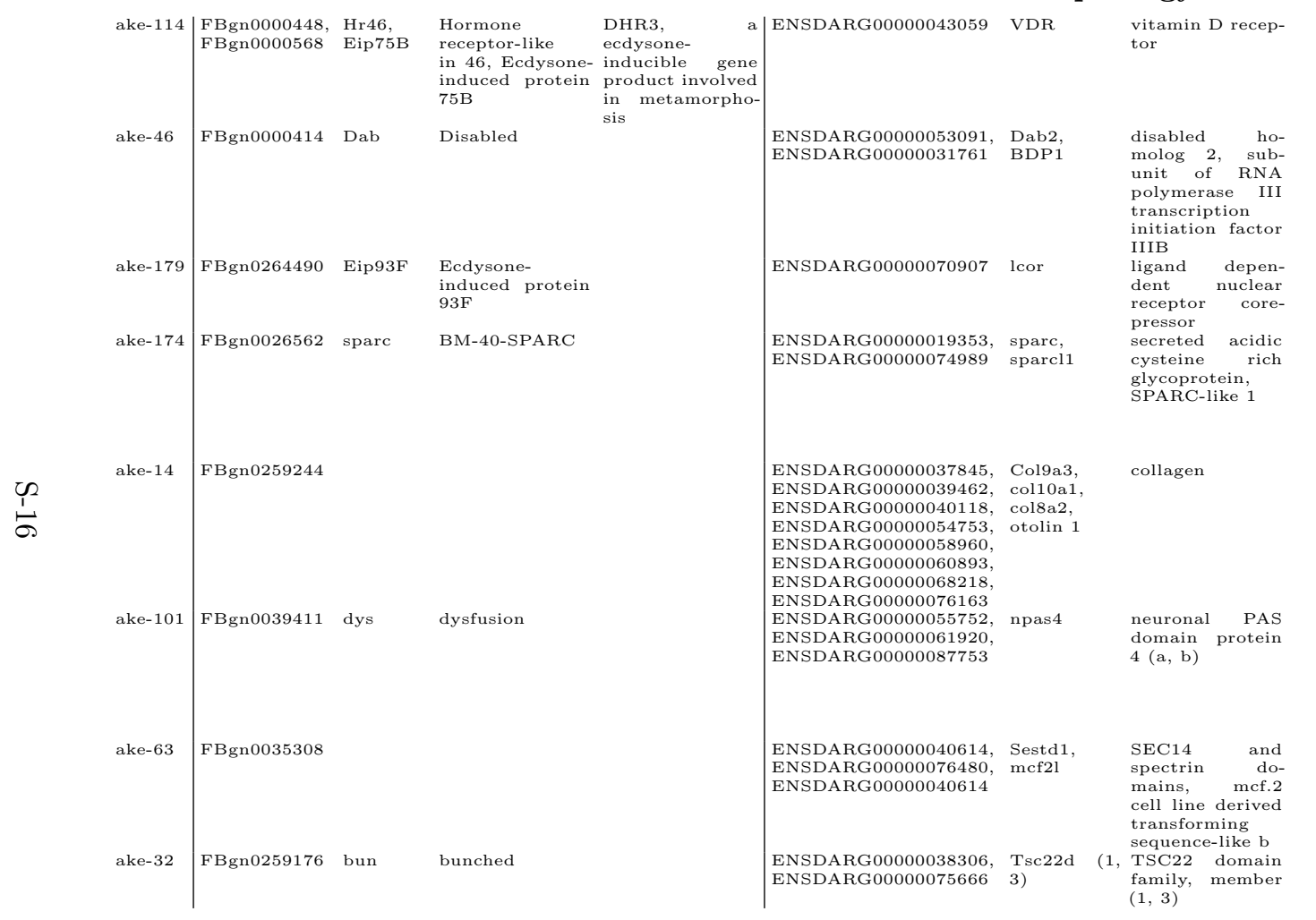

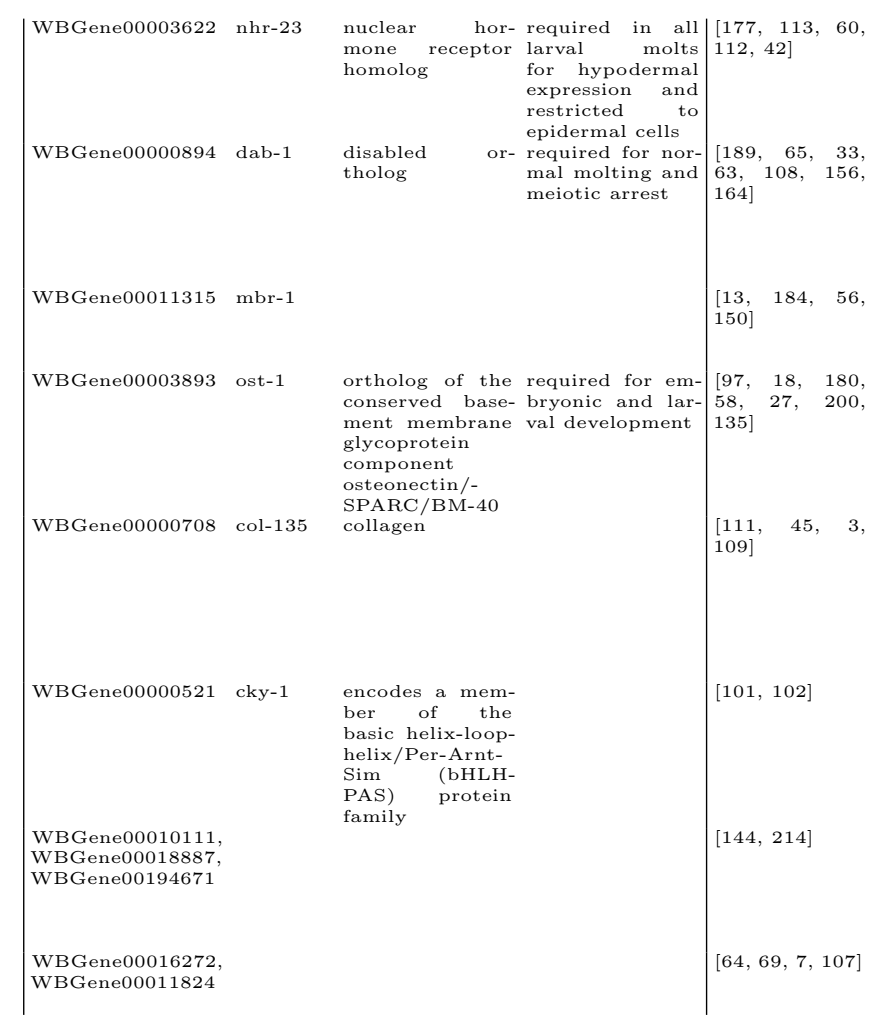

\section{Muscle related}

\begin{tabular}{|c|c|c|c|c|c|}
\hline ake-16 & FBgn0004028 wupA & wings up $\mathrm{A}$ & troponin I $(\mathrm{TnI})$ & \begin{tabular}{|l} 
ENSDARG00000005841, \\
ENSDARG0000013752, \\
ENSDARG0000002906, \\
ENSDARG0000029995, \\
ENSDARG00000035958, \\
ENSDARG0000042559, \\
ENSDARG00000045592, \\
ENSDARG0000052708, \\
ENSDARG00000073766
\end{tabular} & $\begin{array}{l}\operatorname{tnni} \quad \text { (2a.2, troponin I, skele- } \\
2 \mathrm{a} .3, \quad 2 \mathrm{a} .4, \text { tal, fast, tandem } \\
\text { 2b.2, 2b.1, duplicate } \\
\text { 1., 2a.1, 1, } \\
\text { 1d) }\end{array}$ \\
\hline
\end{tabular}


... Table S5 continued

\begin{tabular}{|c|c|c|c|c|c|c|c|c|c|c|c|c|c|}
\hline $\begin{array}{l}\text { internal } \\
\text { ID }\end{array}$ & Flybase ID & Symbol & $\begin{array}{l}\text { Name } \\
\text { Fly }\end{array}$ & Description & Ensembl ID & Symbol & $\begin{array}{l}\text { Name } \\
\text { Fish }\end{array}$ & Description & Wormbase ID & Symbol & $\begin{array}{c}\text { Name } \\
\text { Worm }\end{array}$ & Description & $\begin{array}{l}\text { References in in } \\
\text { Supplemen-n- } \\
\text { tary file } 6\end{array}$ \\
\hline
\end{tabular}

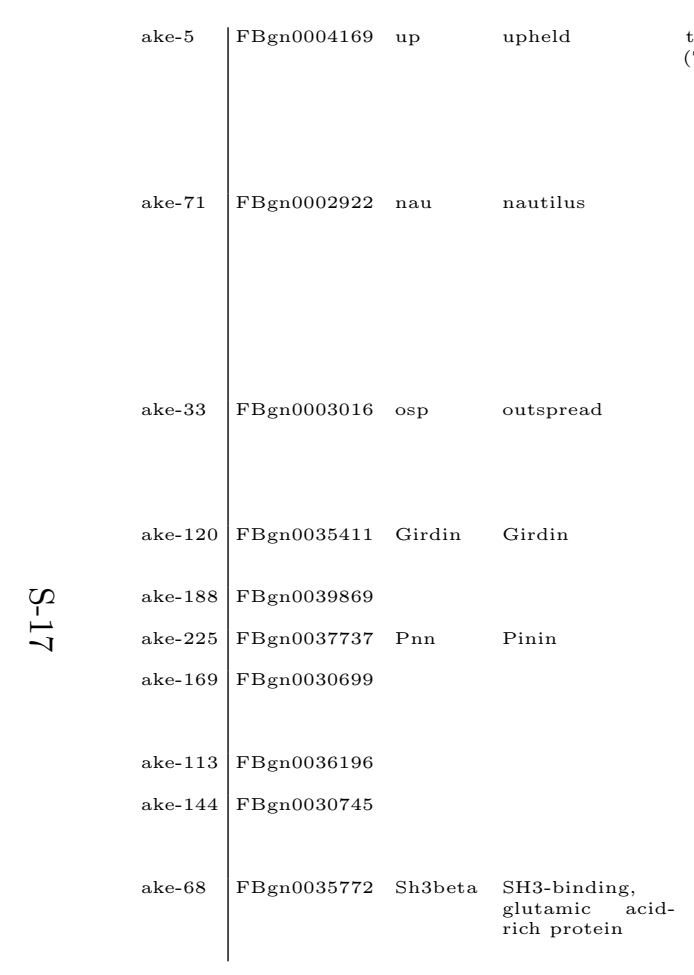

troponin
$(\mathrm{TnT})$

$\begin{array}{ll}\text { ENSDARG00000002988, tnnt } & \text { (2d, Troponin T (2d, } \\ \text { ENSDARG00000030270, 3a, 2e, 3b) } & \text { 2e -cardiac; } 3 \mathrm{a}, \\ \text { ENSDARG00000037954, } & 3 \mathrm{~b} \text {-skeletal; typ } \\ \text { ENSDARG00000045822, } & 1 \text {-skeletal) } \\ \text { ENSDARG00000068457 } & \\ & \\ \text { ENSDARG00000007277, Myf } & (5,6, \text { myogenic factor }\end{array}$ ENSDARG00000029830, d1
ENSDA ENSDARG00000030110 X TRIO and F$\begin{array}{ll}\text { ENSDARG00000074705, } & \begin{array}{l}\text { actin bin, binding } \\ \text { protein, myosin } \\ \text { phosphatase }\end{array}\end{array}$

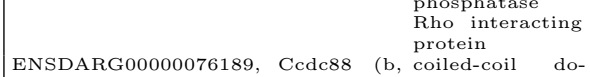
$\begin{array}{llll}\begin{array}{l}\text { ENSDARG00000076189, } \\ \text { ENSDARG00000078440 }\end{array} & \begin{array}{l}\text { Ccdc8 } \\ \text { Aa) }\end{array} & \begin{array}{c}\text { (b, coiled-coil do- } \\ \text { main containing }\end{array} \\ & & \end{array}$ $\begin{array}{lll}\text { ENSDARG } & \text { main containing } \\ \text { ENSDARG00000016754 } & \text { tbca } & \text { (88B, } 88 \text { Aa) } \\ \text { tubulin cofactor }\end{array}$ ENSDARG00000015851 pun ENSDARG00000010400 lrrfip2 $\begin{array}{lll} & \text { interacting pro- } \\ \text { tein } 2 & \\ \text { ENSDARG00000040277, Fbxo } & (32, \text { F-box } & \text { protein }\end{array}$ ENSDARG 000075172 25) $\quad(32,25)$ ENSDARG00000024047, Tmem38a, transmembrane

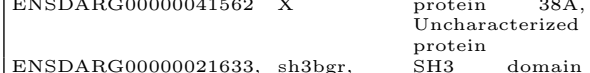
ENSDARG00000036878, sh3bgrl2 binding ry glu-
ENSDARG00000058302

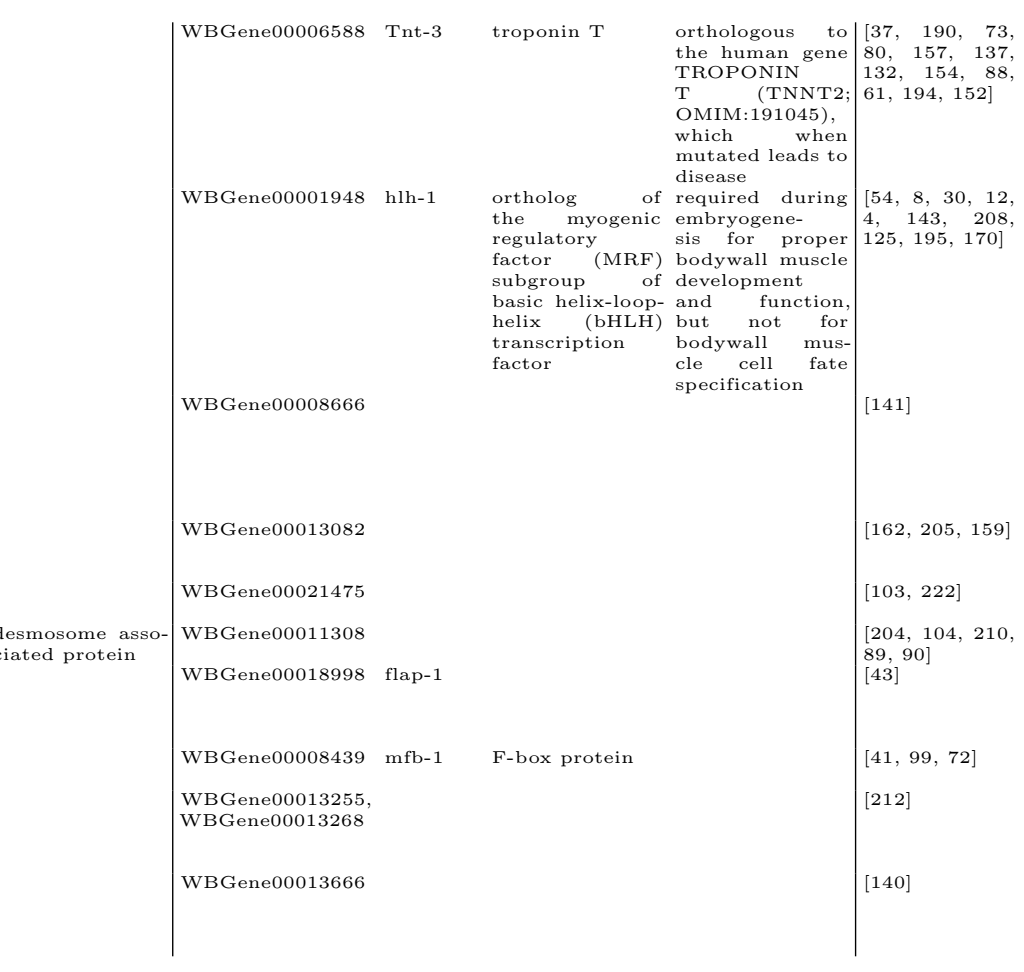

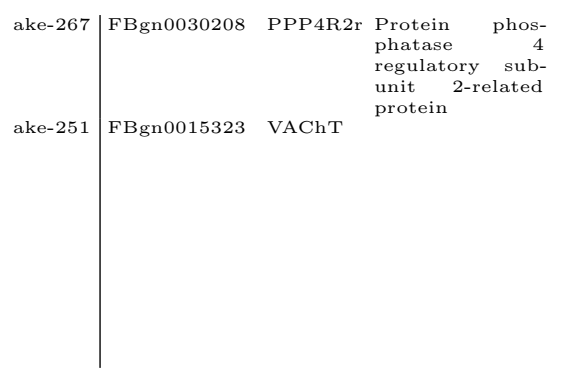

\section{Neuron related}

\begin{tabular}{|c|c|c|c|c|c|c|c|}
\hline ENSDARG00000026540 & ppp4r2a & $\begin{array}{l}\text { protein } \\
\text { phatase } 4 \text { regu- } \\
\text { latory subunit } \\
\text { 2a }\end{array}$ & |WBGene00017064 & Ppfr-2 & & & [17] \\
\hline $\begin{array}{l}\text { ENSDARG00000006356, } \\
\text { ENSDARG00000090189, }\end{array}$ & Slc18a3a, X & 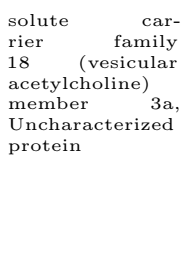 & $\begin{array}{l}\text { WBGene000000481, } \\
\text { WBGene00006756 }\end{array}$ & $\begin{array}{l}\text { Cha-1, } \\
\text { unc-17 }\end{array}$ & $\begin{array}{l}\text { Cha-1 encodes } \\
\text { a choline acetyl- } \\
\text { transferase that } \\
\text { synthesizes } \\
\text { acetylcholine, } \\
\text { unc-17 encodes } \\
\text { a synaptic vesi- } \\
\text { cle acetylcholine } \\
\text { transporter } \\
\text { (VAChT), share } \\
\text { a common } \\
\text { promoter }\end{array}$ & $\begin{array}{l}\text { expressed in } \\
\text { neurons, and } \\
\text { is required for } \\
\text { viability, nor- } \\
\text { mal growth, } \\
\text { locomotion, } \\
\text { and sensitiv- } \\
\text { aty to acetyl- } \\
\text { cholinesterase } \\
\text { inhibitors }\end{array}$ & {$[44,83,51]$} \\
\hline
\end{tabular}


.. Table S5 continued

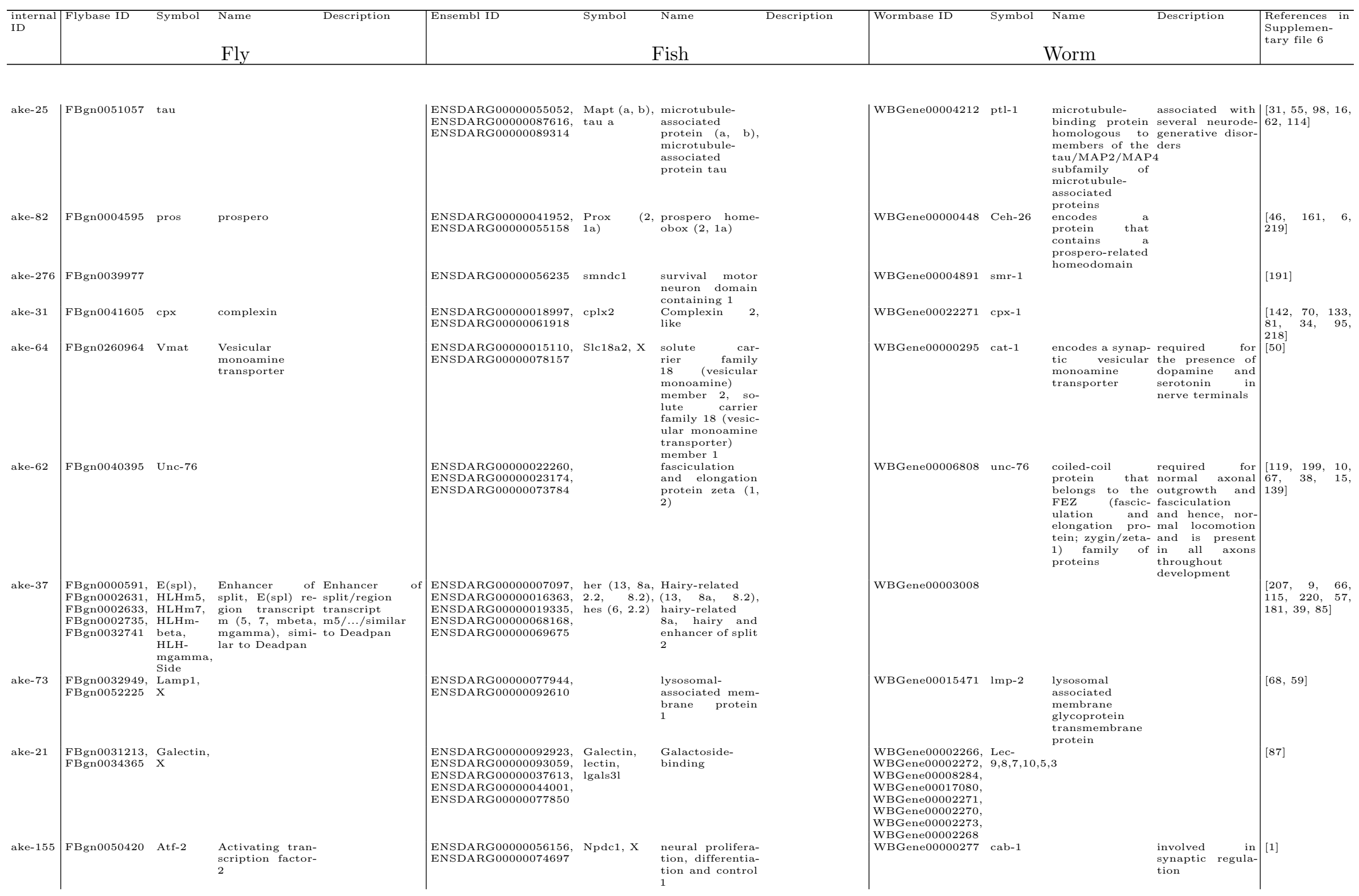


Table S5 continued

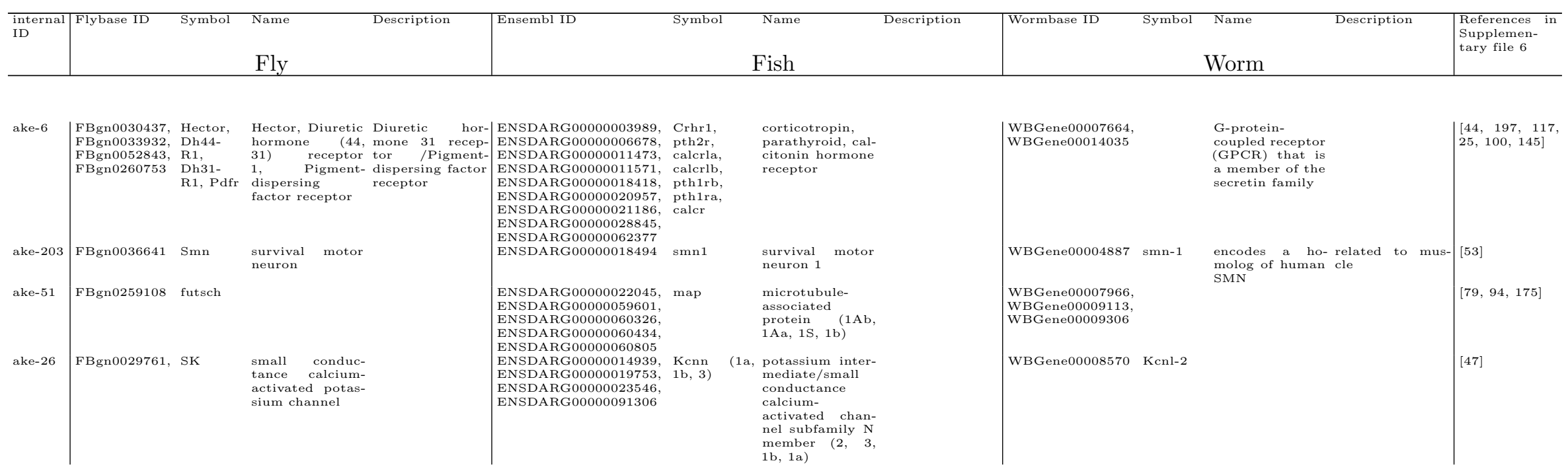


Table S5 continued

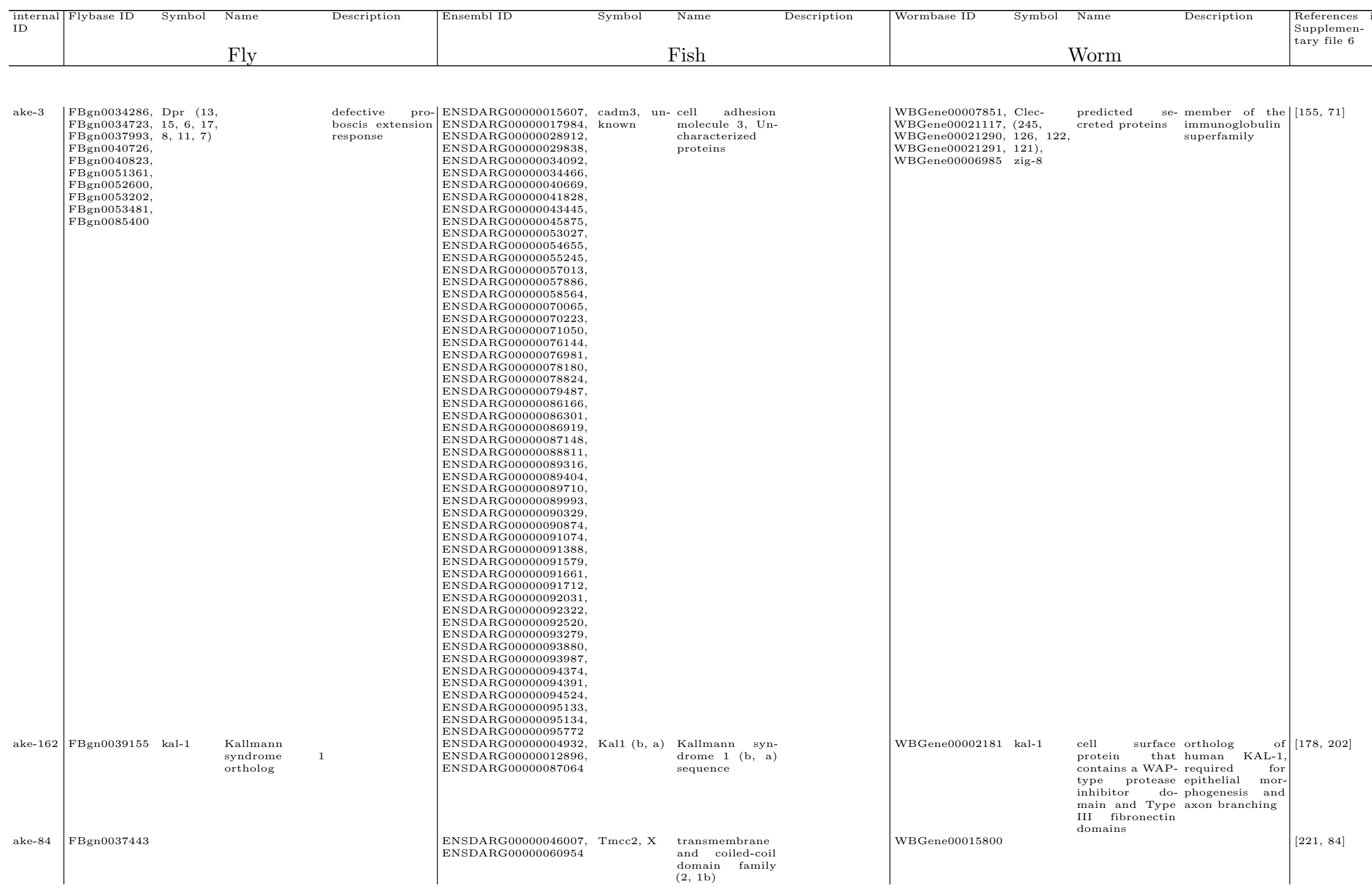


... Table S5 continued

\begin{tabular}{|c|c|c|c|c|c|c|c|c|c|c|c|c|c|}
\hline $\begin{array}{l}\text { internal } \\
\text { ID }\end{array}$ & Flybase ID & Symbol & $\begin{array}{l}\text { Name } \\
\text { Fly }\end{array}$ & Description & Ensembl ID & Symbol & $\begin{array}{l}\text { Name } \\
\text { Fish }\end{array}$ & Description & Wormbase ID & Symbol & $\begin{array}{l}\text { Name } \\
\text { Worm }\end{array}$ & Description & $\begin{array}{l}\text { References in } \\
\text { Supplemen- } \\
\text { tary file } 6\end{array}$ \\
\hline
\end{tabular}

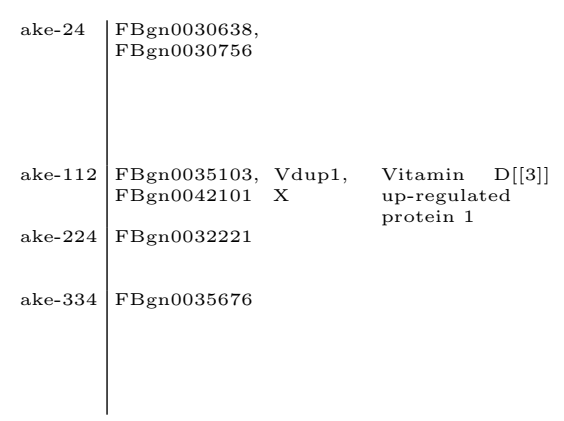

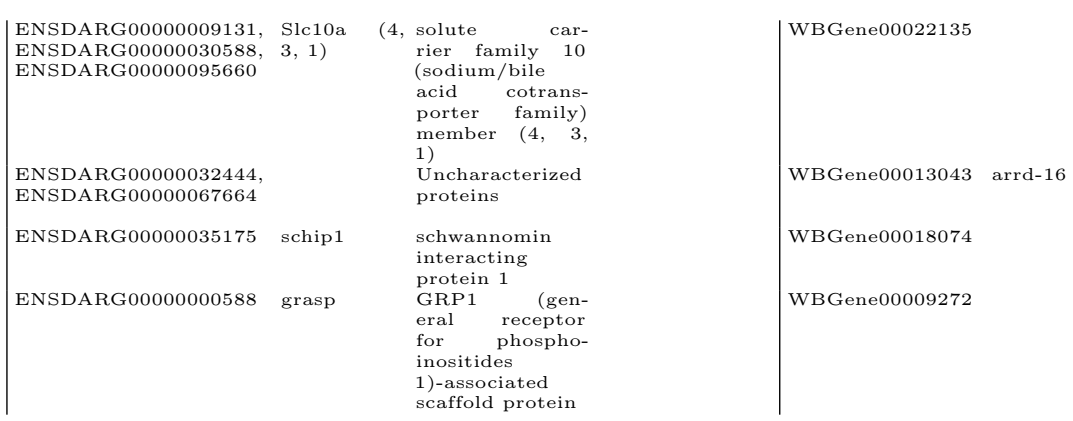

$\mid \begin{aligned} & {[120,167]} \\ & {[123,28]} \\ & {[74,134]} \\ & {[110]}\end{aligned}$

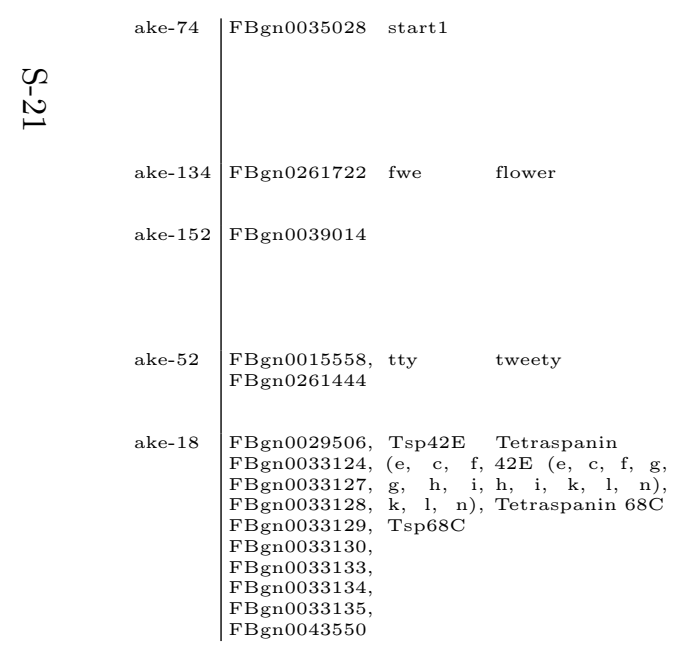

\section{Signalling related}

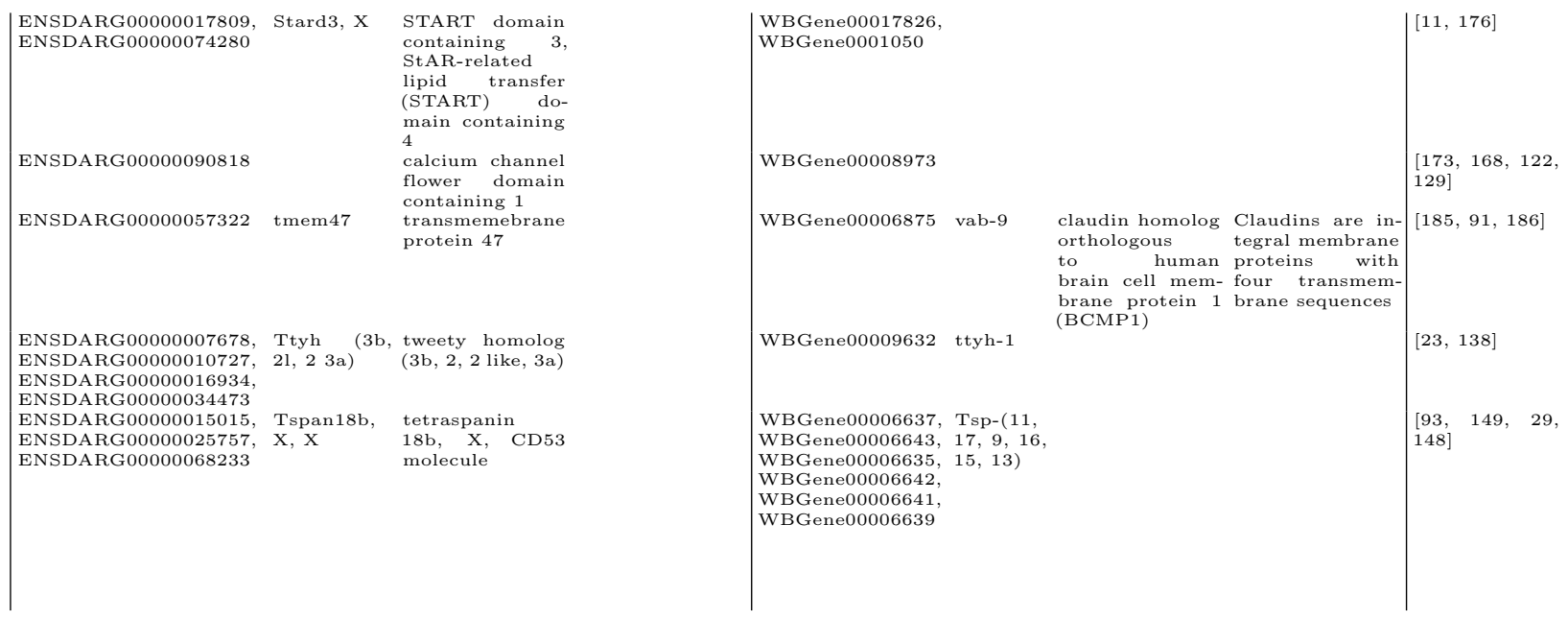


... Table S5 continued

\begin{tabular}{|c|c|c|c|c|c|c|c|c|c|c|c|c|}
\hline $\begin{array}{l}\text { internal } \\
\text { ID }\end{array}$ & Flybase ID & Symbol & $\begin{array}{l}\text { Name } \\
\text { Fly }\end{array}$ & Description & Ensembl ID & Symbol & $\begin{array}{l}\text { Name } \\
\text { Fish }\end{array}$ & Description & Wormbase ID & $\begin{aligned} \text { Symbol } & \text { Name } \\
& \text { Worm }\end{aligned}$ & Description & $\begin{array}{l}\text { References in } \\
\text { Supplemen- } \\
\text { tary file } 6\end{array}$ \\
\hline
\end{tabular}

\begin{tabular}{|c|c|c|}
\hline $\begin{array}{l}\text { ENSDARG00000078331, } \\
\text { ENSDARG00000078455, } \\
\text { ENSDARG00000079163 }\end{array}$ & $\begin{array}{l}\text { Best } \\
2)\end{array}$ & $\begin{array}{l}\text { Bestrophin }(1,4 \text {, } \\
2)\end{array}$ \\
\hline $\begin{array}{l}\text { ENSDARG00000007697, } \\
\text { ENSDARG00000023290, } \\
\text { ENSDARG00000034650, } \\
\text { ENSDARG00000035904, } \\
\text { ENSDARG00000045926 }\end{array}$ & $\begin{array}{ll}\operatorname{Fabp}(7 \mathrm{a}, & 3, \mathrm{f} \\
7 \mathrm{~b}) \text { crabp1 } & \mathrm{i} \\
(\mathrm{b}, \mathrm{a}) & -\mathrm{r} \\
& \mathrm{r} \\
& \mathrm{r} \\
& \mathrm{r} \\
& \end{array}$ & $\begin{array}{l}\text { fatty acid bind- } \\
\text { ing protein ( } 7 \\
-\quad \text { brain, } 3 \\
\text { muscle), cellular } \\
\text { retinoic acid } \\
\text { binding protein } \\
1 \text { (a, b) }\end{array}$ \\
\hline ENSDARG00000056605 & Wbp2 & $\begin{array}{ll}\text { WW } & \text { domain } \\
\text { binding } & \text { protein } \\
2\end{array}$ \\
\hline ENSDARG00000074236 & & \\
\hline ENSDARG00000088272 & $\operatorname{srp} 14$ & $\begin{array}{ll}\text { signal } & \text { recogni- } \\
\text { tion } & \text { particle }\end{array}$ \\
\hline ENSDARG00000021112 & $\mathrm{c} 1 \mathrm{~d}$ & $\begin{array}{l}14 \\
\text { C1D } \\
\text { receptor }\end{array}$ \\
\hline ENSDARG00000042892 & paip1 & $\begin{array}{l}\text { repressor } \\
\text { poly (A) binding } \\
\text { protein interact- } \\
\text { ine protein } 1\end{array}$ \\
\hline $\begin{array}{l}\text { ENSDARG00000010432, } \\
\text { ENSDARG00000070795 }\end{array}$ & $\operatorname{Eaf}(2,1) \quad \begin{array}{l}1 \\
\mathrm{E} \\
\mathrm{f}\end{array}$ & $\begin{array}{l}\text { ng proten } 1 \\
\text { ELL associated } \\
\text { factor }(2,1)\end{array}$ \\
\hline ENSDARG00000059177 & $\operatorname{tax} 1 \mathrm{bp} 3$ & $\begin{array}{lr}\text { Tax1 } & \text { (human } \\
\text { T-cell } & \text { leukemia } \\
\text { virus } & \text { type I I) } \\
\text { binding } & \text { protein } \\
3 & \end{array}$ \\
\hline $\begin{array}{l}\text { ENSDARG00000013655, } \\
\text { ENSDARG0000027154, } \\
\text { ENSDARG00000042548 } \\
\text { ENSDARG000002839, } \\
\text { ENSDARG00000031836, } \\
\text { ENSDARG00000039319 }\end{array}$ & $\begin{array}{lr}\text { Tpd521 } & (1, \mathrm{t} \\
2 \mathrm{~b}, 2 \mathrm{a}) & \mathrm{I} \\
\mathrm{vps} 37 & 1 \\
& \mathrm{v} \\
& \mathrm{s} \\
& \mathrm{r}\end{array}$ & $\begin{array}{l}\text { tumor protein } \\
\text { D52-like }(2 \mathrm{~b}, 2 \mathrm{a}, \\
\text { 1) } \\
\text { vacuolar protein } \\
\text { sorting } 37 \text { ho- } \\
\text { molog }(B, C)\end{array}$ \\
\hline ENSDARG00000070512 & $\operatorname{tnrc5}$ & $\begin{array}{l}\text { trinucleotide re- } \\
\text { peat containing } \\
5\end{array}$ \\
\hline
\end{tabular}

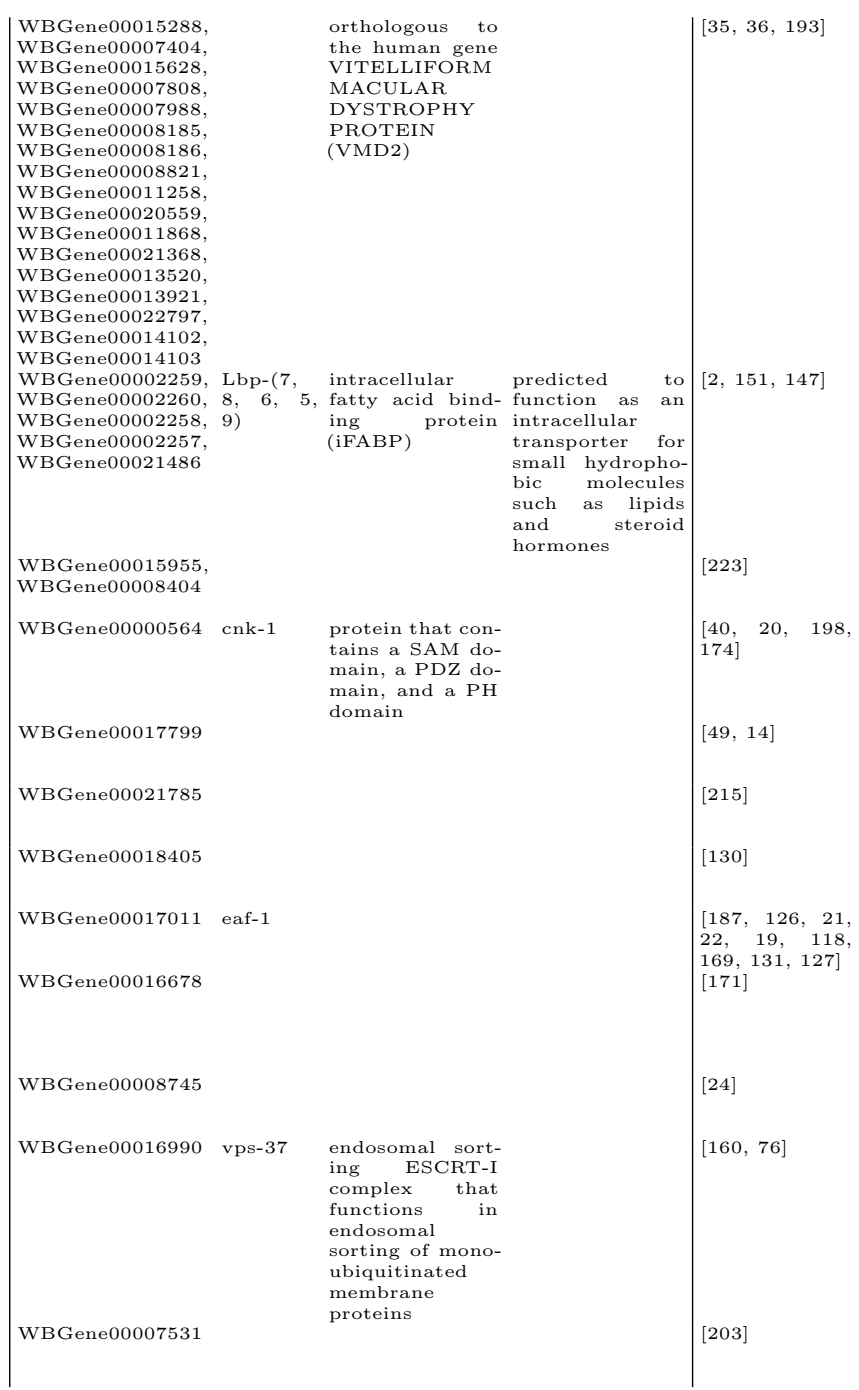


... Table S5 continued

\begin{tabular}{|c|c|c|c|c|c|c|c|c|c|c|c|c|c|}
\hline $\begin{array}{l}\text { internal } \\
\text { ID }\end{array}$ & Flybase ID & Symbol & $\begin{array}{c}\text { Name } \\
\text { Fly }\end{array}$ & Description & Ensembl ID & Symbol & $\begin{array}{l}\text { Name } \\
\text { Fish }\end{array}$ & Description & Wormbase ID & Symbol & $\begin{array}{l}\text { Name } \\
\text { Worm }\end{array}$ & Description & $\begin{array}{l}\text { References in } \\
\text { Supplemen- } \\
\text { tary file } 6\end{array}$ \\
\hline
\end{tabular}

ake-142 |F F Bnn0036090

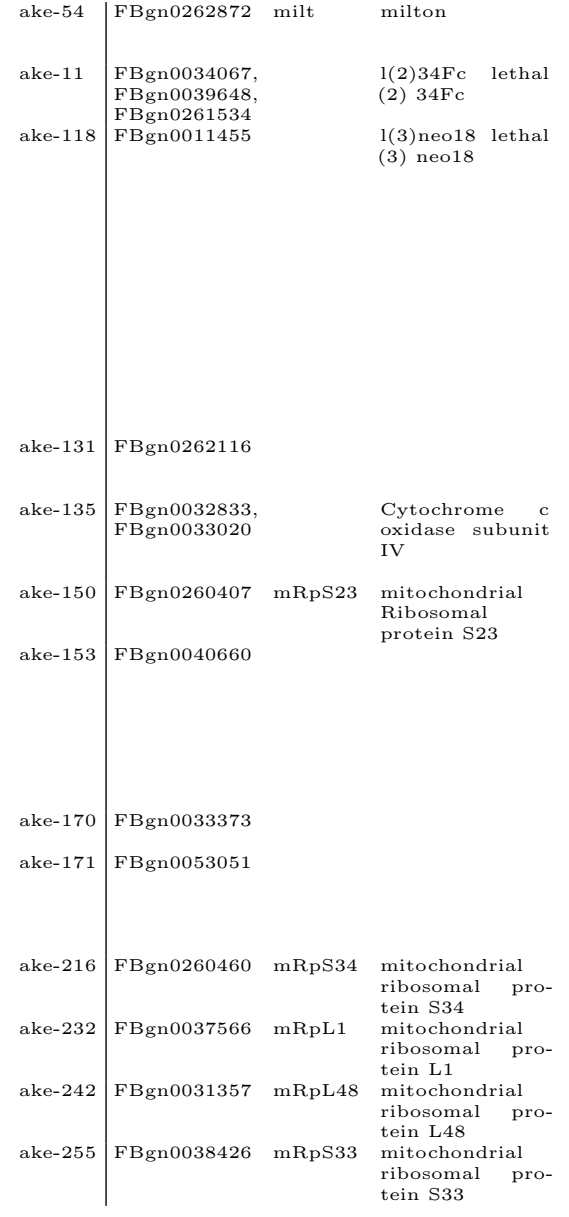

\begin{tabular}{|lll} 
ENSDARG00000036064 & Cnep1r1 & $\begin{array}{l}\text { CTD nuclear } \\
\text { envelope phos- } \\
\text { phatase } 1 \text { regu- } \\
\text { latory subunit }\end{array}$ \\
& & 1
\end{tabular}

Regulation related

\begin{tabular}{|c|c|c|}
\hline \multirow{5}{*}{$\begin{array}{l}\text { ENSDARG00000041304, } \\
\text { ENSDARG00000078872, } \\
\text { ENSDARG00000060622, } \\
\text { ENSDARG0000069103, } \\
\text { ENSDARG00000093957 } \\
\text { ENSDARG00000070824 }\end{array}$} & \multicolumn{2}{|r|}{ lated } \\
\hline & trak 2 & ing pro- \\
\hline & & $\operatorname{ding}(2$, \\
\hline & & $\begin{array}{l}\text { ferrrc-chelate } \\
\text { ductase } 1 \text { like }\end{array}$ \\
\hline & ndufb5 & $\begin{array}{l}\text { NADH de- } \\
\text { hydrogenase } \\
\text { (ubiquinone) } 1 \\
\text { beta subcom- } \\
\text { plex 5 suber }\end{array}$ \\
\hline ENSDARG00000069336, & Rnasek (b, & ribo \\
\hline $\begin{array}{l}\text { ENSDARG00000069336, } \\
\text { ENSDARG00000069461 }\end{array}$ & & $\mathrm{R}$ \\
\hline $\begin{array}{l}\text { ENSDARG00000022509, } \\
\text { ENSDARG00000032970 }\end{array}$ & $\operatorname{Cox} 4 \mathrm{i}(2,1)$ & $\begin{array}{l}\text { Cytochrome c } \\
\text { oxidase subunit } \\
\text { IV isoform }(2,\end{array}$ \\
\hline ENSDARG00000033973 & $\operatorname{Mrps} 23$ & $\begin{array}{l}\text { 1) } \\
\text { mitochondrial } \\
\text { ribosomal pro- } \\
\text { tein } 523\end{array}$ \\
\hline $\begin{array}{l}\text { ENSDARG00000044092, } \\
\text { ENSDARG00000067975 }\end{array}$ & $\begin{array}{l}\text { Atpif1 } \\
\text { a) }\end{array}$ & $\begin{array}{l}\text { ATPase in- } \\
\text { hibitory factor } 1 \\
(\mathrm{~b}, \mathrm{a})\end{array}$ \\
\hline ENSDARG00000007181 & nadk2 & $\begin{array}{l}\text { NAD kinase } 2 \\
\text { mitochondrial }\end{array}$ \\
\hline $\begin{array}{l}\text { ENSDARG00000012044, } \\
\text { ENSDARG00000024687 }\end{array}$ & $\begin{array}{l}\text { polr3gla, } \\
\text { polr3g }\end{array}$ & $\begin{array}{l}\text { polymerase } \\
\text { (RNA) III } \\
\text { (DNA directed) } \\
\text { polypeptide G, } \\
\text { like a }\end{array}$ \\
\hline ENSDARG00000057910 & $\operatorname{mrps} 34$ & $\begin{array}{l}\text { mitochondrial } \\
\text { ribosomal pro- } \\
\text { tein S34 }\end{array}$ \\
\hline ENSDARG00000054606 & mrpl1 & $\begin{array}{l}\text { mitochondrial } \\
\text { ribosomal pro- } \\
\text { tein L1 }\end{array}$ \\
\hline ENSDARG00000035167 & $\operatorname{mrpl} 48$ & $\begin{array}{l}\text { mitochondrial } \\
\text { ribosomal pro- } \\
\text { tein L48 }\end{array}$ \\
\hline ENSDARG0 & $\mathrm{mr}$ & $\begin{array}{l}\text { mitochondrial } \\
\text { ribosomal pro- } \\
\text { tein S33 }\end{array}$ \\
\hline
\end{tabular}

WBGene00011828

[ [77]

WBGene00020838

WBGene00007339,

WBGene00007545,

WBGene0001611

ortholog
of the

of the
NDUFB $5 /$ SGDH

subunit

mito-

chon-
drial
NADH

NADH
dehydro-

genase
(ubiquinone)

(complex

WBGene00016721,

WBGene00012354

theng

WBGene00014224

WBGene00015248 mai-2

orthologous to blocks reverse
mitochondrial action (ATP
intrinsic AT- hydrolysis) (ATP
Pase inhibitor by F(0)F(1)-

orthologous to blocks reverse
mitochondrial action (ATP
intrinsic AT- hydrolysis)
Pase inhibitor by F(0)F(1)-
protein IF(1)) ATPase when

ATPase when
its (normally
required) proton

WBGene00012463

gradient is lost

[86]

WBGene00021638

[206, 172, 209]

WBGene00010905

WBGene00017997

$\left[\begin{array}{ll}{[179,} & 158,26 \\ 136,188] & 26\end{array}\right.$

$[179,158,26$,
$136,188]$

$[179, \quad 158,26$,
$136,188]$

$[179,158,26$,
$136,188]$ 
Table S5 continued

\begin{tabular}{|c|c|c|c|c|c|c|c|c|c|c|c|c|c|}
\hline $\begin{array}{l}\text { internal } \\
\text { ID }\end{array}$ & Flybase ID & Symbol & $\begin{array}{l}\text { Name } \\
\text { Fly }\end{array}$ & Description & Ensembl ID & Symbol & $\begin{array}{l}\text { Name } \\
\text { Fish }\end{array}$ & Description & Wormbase ID & Symbol & $\begin{array}{l}\text { Name } \\
\text { Worm }\end{array}$ & Description & $\begin{array}{l}\text { References in } \\
\text { Supplemen- } \\
\text { tary file } 6\end{array}$ \\
\hline
\end{tabular}

\begin{tabular}{l|lll} 
ake-259 & FBgn0032053 & mRpL51 & $\begin{array}{l}\text { mitochondrial } \\
\text { ribosomal } \\
\text { tein L51 }\end{array}$ \\
ake-97 & FBgn0034986 & mRpS17 & $\begin{array}{l}\text { mitochondrial } \\
\text { ribosomal } \\
\text { tein S17 }\end{array}$ \\
ake-327 & FBgn006411 & & \\
ake-160 & FBgn0038400 & & \\
ake-217 & FBgn0025820 & JTBR & \\
ake-323 & FBgn0037700 & & \\
ake-193 & FBgn0032200 &
\end{tabular}

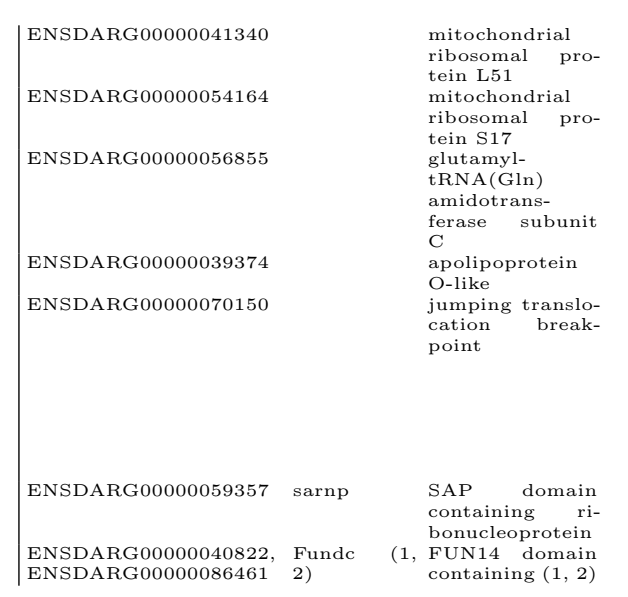

$\mid \begin{aligned} & \text { WBGene00011740 } \\ & \text { WBGene00015487 } \\ & \text { WBGene00013433 } \\ & \text { WBGene00019333 moma-1 } \\ & \text { WBGene00002180 jtr-1 } \\ & \text { WBGene00021813 } \\ & \text { WBGene00011528 }\end{aligned}$

\begin{tabular}{l|l} 
& $\mid \begin{array}{ll}179, \quad 158, \quad 26, \\
136,188] \\
{[179,158, \quad 26,} \\
136,188] \\
{[52,153]}\end{array}$ \\
\\
{$[78]$} \\
$\begin{array}{l}\text { predicted trans- } \\
\text { membrane } \\
\text { protein that is } \\
\text { related to the } \\
\text { conserved jump- } \\
\text { ing transloca- } \\
\text { tion breakpoint } \\
\text { (JTB) family of } \\
\text { proteins }\end{array}$ & {$[105]$} \\
& \\
& {$[116,213]$} \\
& {$[211,128]$}
\end{tabular}

\begin{tabular}{l|l} 
ake-184 & FBgn0031413 \\
ake-185 & FBgn0032488
\end{tabular}

ENSDARG00000086655,
ENSDARG00000089374
ENSDARG00000020611

\section{Others}

\begin{tabular}{l|l} 
chromosome 3 & WBGene00021131 \\
open reading \\
frame 83 \\
chromosome 19 \\
Open reading \\
frame 47
\end{tabular}


. Table S5 continued

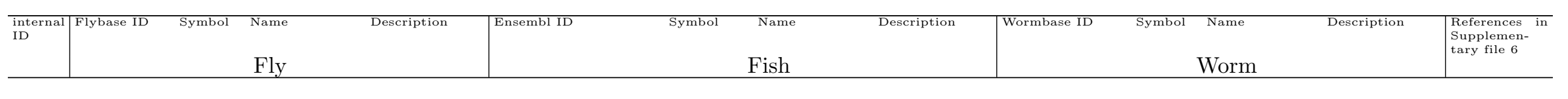
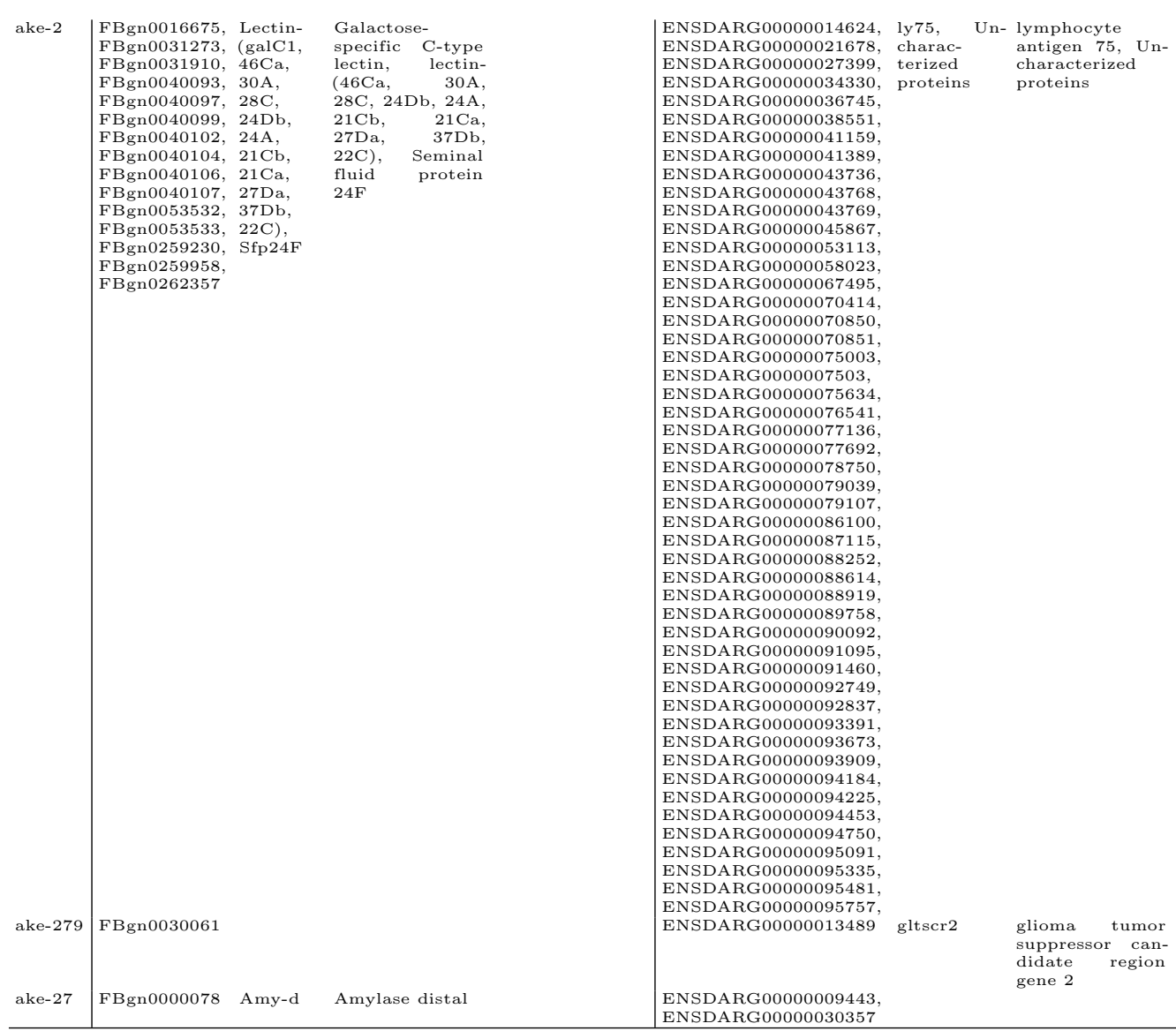

WBGene00015631, Clec-(89,
WBGene00009517, 167, 166,

WBGene00009517, $167, \quad 166$,
WBGene00009518, $172, \quad 209$

WBGene00018910, $174, \quad 83$,
WBGene00044719, $84, \quad 210$
WBGenos

WBGene00044719, $84, \quad 210$,
WBGene00021224, 187, 186)

WBGene00021580,

WBGene00021879,
WBGene00021895,

WBGene00021895,
WBGene00023484,

WBGene00022261,

WBGene00014137,

WBGene0001413

(1) 
bioRxiv preprint doi: https://doi.org/10.1101/786723; this version posted September 30, 2019. The copyright holder for this preprint (which was not certified by peer review) is the author/funder, who has granted bioRxiv a license to display the preprint in perpetuity. It is made available

\section{References for Supplementary Material}

1. J. Ackermann, G. Ashton, S. Lyons, D. James, J.P. Hornung, N. Jones, and W. Breitwieser. Loss of ATF2 function leads to cranial motoneuron degeneration during embryonic mouse development. PLoS One, 6(4):e19090, 2011.

2. H.J. Ahn, S.H. Jeon, and S.H. Kim. Expression of a set of glial cell-specific markers in the Drosophila embryonic central nervous system. BMB Rep, 47(6):354-359, Jun 2014.

3. D. Aldea, P. Hanna, D. Munoz, J. Espinoza, M. Torrejon, L. Sachs, N. Buisine, S. Oulion, H. Escriva, and S. Marcellini. Evolution of the vertebrate bone matrix: an expression analysis of the network forming collagen paralogues in amphibian osteoblasts. J Exp Zool B Mol Dev Evol, 320(6):375-384, Sep 2013.

4. A.A. Amali, C.J. Lin, Y.H. Chen, W.L. Wang, H.Y. Gong, C.Y. Lee, Y.L. Ko, J.K. Lu, G.M. Her, T.T. Chen, and J.L. Wu. Up-regulation of muscle-specific transcription factors during embryonic somitogenesis of zebrafish (Danio rerio) by knock-down of myostatin-1. Dev Dyn, 229(4):847-856, Apr 2004.

5. S. Aras, O. Pak, N. Sommer, R. Finley, Jr., M. Huttemann, N. Weissmann, and L.I. Grossman. Oxygen-dependent expression of cytochrome c oxidase subunit 4-2 gene expression is mediated by transcription factors RBPJ, CXXC5 and CHCHD2. Nucleic Acids Res, 41(4):2255-2266, Feb 2013.

6. C.L. Araya, T. Kawli, A. Kundaje, L. Jiang, B. Wu, D. Vafeados, R. Terrell, P. Weissdepp, L. Gevirtzman, D. Mace, W. Niu, A.P. Boyle, D. Xie, L. Ma, J.I. Murray, V. Reinke, R.H. Waterston, and M. Snyder. Regulatory analysis of the C. elegans genome with spatiotemporal resolution. Nature, 512(7515):400-405, Aug 2014.

7. D.M. Ash, J.F. Hackney, M. Jean-Francois, N.C. Burton, and L.L. Dobens. A dominant negative allele of the Drosophila leucine zipper protein Bunched blocks bunched function during tissue patterning. Mech Dev, 124(7-8):559-569, Aug 2007.

8. A. Aziz, Q.C. Liu, and F.J. Dilworth. Regulating a master regulator: establishing tissue-specific gene expression in skeletal muscle. Epigenetics, 5(8):691-695, Nov 2010.

9. S. Bae, Y. Bessho, M. Hojo, and R. Kageyama. The bHLH gene Hes6, an inhibitor of Hes1, promotes neuronal differentiation. Development, 127(13):2933-2943, Jul 2000.

10. B.J. Barsi-Rhyne, K.M. Miller, C.T. Vargas, A.B. Thomas, J. Park, M. Bremer, J.L. Jarecki, and M.K. VanHoven. Kinesin-1 acts with netrin and DCC to maintain sensory neuron position in Caenorhabditis elegans. Genetics, 194(1):175-187, May 2013.

11. M.P. Bauer, J.T. Bridgham, D.M. Langenau, A.L. Johnson, and F.W. Goetz. Conservation of steroidogenic acute regulatory (StAR) protein structure and expression in vertebrates. Mol Cell Endocrinol, 168(1-2):119-125, Oct 2000.

12. L.R. Baugh and C.P. Hunter. Myod, modularity, and myogenesis: conservation of regulators and redundancy in C. elegans. Genes Dev, 20(24):3342-3346, Dec 2006.

13. M. Bender, F.B. Imam, W.S. Talbot, B. Ganetzky, and D.S. Hogness. Drosophila ecdysone receptor mutations reveal functional differences among receptor isoforms. Cell, 91(6):777-788, Dec 1997.

14. M. Blank and T. Burmester. Widespread occurrence of N-terminal acylation in animal globins and possible origin of respiratory globins from a membrane-bound ancestor. Mol Biol Evol, 29(11):35533561, Nov 2012.

15. L. Bloom and H.R. Horvitz. The Caenorhabditis elegans gene unc-76 and its human homologs define a new gene family involved in axonal outgrowth and fasciculation. Proc Natl Acad Sci USA, 94(7):3414-3419, Apr 1997.

16. B.J. Bolkan and D. Kretzschmar. Loss of tau results in defects in photoreceptor development and progressive neuronal degeneration in Drosophila. Dev Neurobiol, 74(12):1210-1225, Dec 2014.

17. Y. Bosio, G. Berto, P. Camera, F. Bianchi, C. Ambrogio, P. Claus, and F. Di Cunto. PPP4R2 regulates neuronal cell differentiation and survival, functionally cooperating with SMN. Eur J Cell Biol, 91(8):662-674, Aug 2012. 
bioRxiv preprint doi: https://doi.org/10.1101/786723; this version posted September 30, 2019. The copyright holder for this preprint (which was not certified by peer review) is the author/funder, who has granted bioRxiv a license to display the preprint in perpetuity. It is made available under aCC-BY-NC-ND 4.0 International license.

18. A.D. Bradshaw and E.H. Sage. SPARC, a matricellular protein that functions in cellular differentiation and tissue response to injury. J Clin Invest, 107(9):1049-1054, May 2001.

19. D.J. Burgess. Gene expression: Time flies thanks to Pol II pausing. Nat Rev Genet, 14(7):441, Jul 2013.

20. C. Cabernard and M. Affolter. Distinct roles for two receptor tyrosine kinases in epithelial branching morphogenesis in Drosophila. Dev Cell, 9(6):831-842, Dec 2005.

21. L. Cai, B.L. Phong, A.L. Fisher, and Z. Wang. Regulation of fertility, survival, and cuticle collagen function by the Caenorhabditis elegans eaf-1 and ell-1 genes. J Biol Chem, 286(41):35915-35921, Oct 2011.

22. L. Cai, D. Wang, A.L. Fisher, and Z. Wang. Identification of a genetic interaction between the tumor suppressor EAF2 and the retinoblastoma protein ( $\mathrm{Rb})$ signaling pathway in C. elegans and prostate cancer cells. Biochem Biophys Res Commun, 447(2):292-298, May 2014.

23. H.D. Campbell, M. Kamei, C. Claudianos, E. Woollatt, G.R. Sutherland, Y. Suzuki, M. Hida, S. Sugano, and I.G. Young. Human and mouse homologues of the Drosophila melanogaster tweety (tty) gene: a novel gene family encoding predicted transmembrane proteins. Genomics, 68(1):89-92, Aug 2000.

24. Q. Cao, J. Chen, L. Zhu, Y. Liu, Z. Zhou, J. Sha, S. Wang, and J. Li. A testis-specific and testis developmentally regulated tumor protein D52 (TPD52)-like protein TPD52L3/hD55 interacts with TPD52 family proteins. Biochem Biophys Res Commun, 344(3):798-806, Jun 2006.

25. J.C. Cardoso, R.C. Felix, and D.M. Power. Nematode and arthropod genomes provide new insights into the evolution of class 2 B1 GPCRs. PLoS One, 9(3):e92220, 2014.

26. M.E. Casad, D. Abraham, I.M. Kim, S. Frangakis, B. Dong, N. Lin, M.J. Wolf, and H.A. Rockman. Cardiomyopathy is associated with ribosomal protein gene haplo-insufficiency in Drosophila melanogaster. Genetics, 189(3):861-870, Nov 2011.

27. R.M. Ceinos, E. Torres-Nunez, R. Chamorro, B. Novoa, A. Figueras, N.M. Ruane, and J. Rotllant. Critical role of the matricellular protein SPARC in mediating erythroid progenitor cell development in zebrafish. Cells Tissues Organs, 197(3):196-208, 2013.

28. S. Chang, N.V. Mandalaywala, R.G. Snyder, M.C. Levendusky, and R.E. Dearborn, Jr. Hedgehogdependent down-regulation of the tumor suppressor, vitamin D3 up-regulated protein 1 (VDUP1), precedes lamina development in Drosophila. Brain Res, 1324:1-13, Apr 2010.

29. S. Charrin, S. Jouannet, C. Boucheix, and E. Rubinstein. Tetraspanins at a glance. J Cell Sci, 127(Pt 17):3641-3648, Sep 2014.

30. L. Chen, M. Krause, M. Sepanski, and A. Fire. The Caenorhabditis elegans MYOD homologue HLH-1 is essential for proper muscle function and complete morphogenesis. Development, 120(6):1631-1641, Jun 1994.

31. M. Chen, R.N. Martins, and M. Lardelli. Complex splicing and neural expression of duplicated tau genes in zebrafish embryos. J Alzheimers Dis, 18(2):305-317, 2009.

32. W.W. Chen, K. Birsoy, M.M. Mihaylova, H. Snitkin, I. Stasinski, B. Yucel, E.C. Bayraktar, J.E. Carette, C.B. Clish, T.R. Brummelkamp, D.D. Sabatini, and D.M. Sabatini. Inhibition of ATPIF1 ameliorates severe mitochondrial respiratory chain dysfunction in mammalian cells. Cell Rep, 7(1):2734, Apr 2014.

33. H. Cheng, J.A. Govindan, and D. Greenstein. Regulated trafficking of the MSP/Eph receptor during oocyte meiotic maturation in C. elegans. Curr Biol, 18(10):705-714, May 2008.

34. M.C. Chicka and E.R. Chapman. Concurrent binding of complexin and synaptotagmin to liposomeembedded SNARE complexes. Biochemistry, 48(4):657-659, Feb 2009.

35. L.T. Chien and H.C. Hartzell. Drosophila bestrophin-1 chloride current is dually regulated by calcium and cell volume. J Gen Physiol, 130(5):513-524, Nov 2007. 
bioRxiv preprint doi: https://doi.org/10.1101/786723; this version posted September 30, 2019. The copyright holder for this preprint (which was not certified by peer review) is the author/funder, who has granted bioRxiv a license to display the preprint in perpetuity. It is made available under aCC-BY-NC-ND 4.0 International license.

36. L.T. Chien and H.C. Hartzell. Rescue of volume-regulated anion current by bestrophin mutants with altered charge selectivity. J Gen Physiol, 132(5):537-546, Nov 2008.

37. M. Chiodin, J.G. Achatz, A. Wanninger, and P. Martinez. Molecular architecture of muscles in an acoel and its evolutionary implications. J Exp Zool B Mol Dev Evol, 316(6):427-439, Sep 2011.

38. J.J. Chua, E. Butkevich, J.M. Worseck, M. Kittelmann, M. Gronborg, E. Behrmann, U. Stelzl, N.J. Pavlos, M.M. Lalowski, S. Eimer, E.E. Wanker, D.R. Klopfenstein, and R. Jahn. Phosphorylationregulated axonal dependent transport of syntaxin 1 is mediated by a Kinesin-1 adapter. Proc Natl Acad Sci U S A, 109(15):5862-5867, Apr 2012.

39. P.C. Chung, W.S. Lin, P.J. Scotting, F.Y. Hsieh, H.L. Wu, and Y.C. Cheng. Zebrafish Her8a is activated by $\mathrm{Su}(\mathrm{H})$-dependent Notch signaling and is essential for the inhibition of neurogenesis. PLoS One, 6(4):e19394, 2011.

40. A. Claperon and M. Therrien. KSR and CNK: two scaffolds regulating RAS-mediated RAF activation. Oncogene, 26(22):3143-3158, May 2007.

41. B.M. Cleveland and J.P. Evenhuis. Molecular characterization of atrogin-1/F-box protein-32 (FBXO32) and F-box protein-25 (FBXO25) in rainbow trout (Oncorhynchus mykiss): Expression across tissues in response to feed deprivation. Comp Biochem Physiol B Biochem Mol Biol, 157(3):248-257, Nov 2010.

42. T.A. Craig, S. Sommer, C.R. Sussman, J.P. Grande, and R. Kumar. Expression and regulation of the vitamin D receptor in the zebrafish, Danio rerio. J Bone Miner Res, 23(9):1486-1496, Sep 2008.

43. P. Dai, S.Y. Jeong, Y. Yu, T. Leng, W. Wu, L. Xie, and X. Chen. Modulation of TLR signaling by multiple MyD88-interacting partners including leucine-rich repeat Fli-I-interacting proteins. $J$ Immunol, 182(6):3450-3460, Mar 2009.

44. A.S. Denes, G. Jekely, P.R. Steinmetz, F. Raible, H. Snyman, B. Prud'homme, D.E. Ferrier, G. Balavoine, and D. Arendt. Molecular architecture of annelid nerve cord supports common origin of nervous system centralization in bilateria. Cell, 129(2):277-288, Apr 2007.

45. T. Dickmeis, C. Plessy, S. Rastegar, P. Aanstad, R. Herwig, F. Chalmel, N. Fischer, and U. Strahle. Expression profiling and comparative genomics identify a conserved regulatory region controlling midline expression in the zebrafish embryo. Genome Res, 14(2):228-238, Feb 2004.

46. C.Q. Doe, Q. Chu-LaGraff, D.M. Wright, and M.P. Scott. The prospero gene specifies cell fates in the Drosophila central nervous system. Cell, 65(3):451-464, May 1991.

47. A.M. Dolga, A. de Andrade, L. Meissner, H.G. Knaus, M. Hollerhage, P. Christophersen, H. Zischka, N. Plesnila, G.U. Hoglinger, and C. Culmsee. Subcellular expression and neuroprotective effects of SK channels in human dopaminergic neurons. Cell Death Dis, 5:e999, 2014.

48. T. Domazet-Loso and D. Tautz. A phylogenetically based transcriptome age index mirrors ontogenetic divergence patterns. Nature, 468(7325):815-818, Dec 2010.

49. J. Droge and W. Makalowski. Phylogenetic analysis reveals wide distribution of globin X. Biol Direct, 6:54, 2011.

50. J.S. Duerr, D.L. Frisby, J. Gaskin, A. Duke, K. Asermely, D. Huddleston, L.E. Eiden, and J.B. Rand. The cat-1 gene of Caenorhabditis elegans encodes a vesicular monoamine transporter required for specific monoamine-dependent behaviors. J Neurosci, 19(1):72-84, Jan 1999.

51. J.S. Duerr, H.P. Han, S.D. Fields, and J.B. Rand. Identification of major classes of cholinergic neurons in the nematode Caenorhabditis elegans. J Comp Neurol, 506(3):398-408, Jan 2008.

52. L. Echevarria, P. Clemente, R. Hernandez-Sierra, M.E. Gallardo, M.A. Fernandez-Moreno, and R. Garesse. Glutamyl-tRNAGln amidotransferase is essential for mammalian mitochondrial translation in vivo. Biochem J, 460(1):91-101, May 2014.

53. B.M. Edens, S. Ajroud-Driss, L. Ma, and Y.C. Ma. Molecular mechanisms and animal models of spinal muscular atrophy. Biochim Biophys Acta, Aug 2014. 
bioRxiv preprint doi: https://doi.org/10.1101/786723; this version posted September 30, 2019. The copyright holder for this preprint (which was not certified by peer review) is the author/funder, who has granted bioRxiv a license to display the preprint in perpetuity. It is made available under aCC-BY-NC-ND 4.0 International license.

54. D.G. Edmondson and E.N. Olson. A gene with homology to the myc similarity region of MyoD1 is expressed during myogenesis and is sufficient to activate the muscle differentiation program. Genes Dev, 3(5):628-640, May 1989.

55. C. Fatouros, G.J. Pir, J. Biernat, S.P. Koushika, E. Mandelkow, E.M. Mandelkow, E. Schmidt, and R. Baumeister. Inhibition of tau aggregation in a novel Caenorhabditis elegans model of tauopathy mitigates proteotoxicity. Hum Mol Genet, 21(16):3587-3603, Aug 2012.

56. I. Fernandes, Y. Bastien, T. Wai, K. Nygard, R. Lin, O. Cormier, H.S. Lee, F. Eng, N.R. Bertos, N. Pelletier, S. Mader, V.K. Han, X.J. Yang, and J.H. White. Ligand-dependent nuclear receptor corepressor LCoR functions by histone deacetylase-dependent and -independent mechanisms. Mol Cell, 11(1):139-150, Jan 2003.

57. A.L. Fisher and M. Caudy. Groucho proteins: transcriptional corepressors for specific subsets of DNA-binding transcription factors in vertebrates and invertebrates. Genes Dev, 12(13):1931-1940, Jul 1998.

58. M.C. Fitzgerald and J.E. Schwarzbauer. Importance of the basement membrane protein SPARC for viability and fertility in Caenorhabditis elegans. Curr Biol, 8(23):1285-1288, Nov 1998.

59. M.L. Florez-McClure, L.A. Hohsfield, G. Fonte, M.T. Bealor, and C.D. Link. Decreased insulin-receptor signaling promotes the autophagic degradation of beta-amyloid peptide in C. elegans. Autophagy, 3(6):569-580, Nov 2007.

60. A.R. Frand, S. Russel, and G. Ruvkun. Functional genomic analysis of C. elegans molting. PLoS Biol, 3(10):e312, Oct 2005.

61. C.Y. Fu, H.C. Lee, and H.J. Tsai. The molecular structures and expression patterns of zebrafish troponin I genes. Gene Expr Patterns, 9(5):348-356, Jun 2009.

62. T.A. Fulga, I. Elson-Schwab, V. Khurana, M.L. Steinhilb, T.L. Spires, B.T. Hyman, and M.B. Feany. Abnormal bundling and accumulation of f-actin mediates tau-induced neuronal degeneration in vivo. Nat Cell Biol, 9(2):139-148, Feb 2007.

63. S.E. George, K. Simokat, J. Hardin, and A.D. Chisholm. The VAB-1 Eph receptor tyrosine kinase functions in neural and epithelial morphogenesis in C. elegans. Cell, 92(5):633-643, Mar 1998.

64. A.R. Gerhold, D.J. Richter, A.S. Yu, and I.K. Hariharan. Identification and characterization of genes required for compensatory growth in Drosophila. Genetics, 189(4):1309-1326, Dec 2011.

65. F.B. Gertler, K.K. Hill, M.J. Clark, and F.M. Hoffmann. Dosage-sensitive modifiers of Drosophila abl tyrosine kinase function: prospero, a regulator of axonal outgrowth, and disabled, a novel tyrosine kinase substrate. Genes Dev, 7(3):441-453, Mar 1993.

66. N. Giagtzoglou, P. Alifragis, K.A. Koumbanakis, and C. Delidakis. Two modes of recruitment of E(spl) repressors onto target genes. Development, 130(2):259-270, Jan 2003.

67. J.G. Gindhart, J. Chen, M. Faulkner, R. Gandhi, K. Doerner, T. Wisniewski, and A. Nandlestadt. The kinesin-associated protein UNC-76 is required for axonal transport in the Drosophila nervous system. Mol Biol Cell, 14(8):3356-3365, Aug 2003.

68. V. Ginet, M.P. Pittet, C. Rummel, M.C. Osterheld, R. Meuli, P.G. Clarke, J. Puyal, and A.C. Truttmann. Dying neurons in thalamus of asphyxiated term newborns and rats are autophagic. Ann Neurol, 76(5):695-711, Nov 2014.

69. S. Gluderer, E. Brunner, M. Germann, V. Jovaisaite, C. Li, C.A. Rentsch, E. Hafen, and H. Stocker. Madm (Mlf1 adapter molecule) cooperates with Bunched A to promote growth in Drosophila. J Biol, $9(1): 9,2010$.

70. D. Glynn, R.J. Sizemore, and A.J. Morton. Early motor development is abnormal in complexin 1 knockout mice. Neurobiol Dis, 25(3):483-495, Mar 2007.

71. T.D. Goldman and M.N. Arbeitman. Genomic and functional studies of Drosophila sex hierarchy regulated gene expression in adult head and nervous system tissues. PLoS Genet, 3(11):e216, Nov 2007. 
bioRxiv preprint doi: https://doi.org/10.1101/786723; this version posted September 30, 2019. The copyright holder for this preprint (which was not certified by peer review) is the author/funder, who has granted bioRxiv a license to display the preprint in perpetuity. It is made available under aCC-BY-NC-ND 4.0 International license.

72. M.D. Gomes, S.H. Lecker, R.T. Jagoe, A. Navon, and A.L. Goldberg. Atrogin-1, a muscle-specific F-box protein highly expressed during muscle atrophy. Proc Natl Acad Sci U S A, 98(25):14440-14445, Dec 2001.

73. A.M. Gordon, E. Homsher, and M. Regnier. Regulation of contraction in striated muscle. Physiol Rev, 80(2):853-924, Apr 2000.

74. L. Goutebroze, E. Brault, C. Muchardt, J. Camonis, and G. Thomas. Cloning and characterization of SCHIP-1, a novel protein interacting specifically with spliced isoforms and naturally occurring mutant NF2 proteins. Mol Cell Biol, 20(5):1699-1712, Mar 2000.

75. B.R. Graveley, A.N. Brooks, J.W. Carlson, M.O. Duff, J.M. Landolin, L. Yang, C.G. Artieri, M.J. van Baren, N. Boley, B.W. Booth, J.B. Brown, L. Cherbas, C.A. Davis, A. Dobin, R. Li, W. Lin, J.H. Malone, N.R. Mattiuzzo, D. Miller, D. Sturgill, B.B. Tuch, C. Zaleski, D. Zhang, M. Blanchette, S. Dudoit, B. Eads, R.E. Green, A. Hammonds, L. Jiang, P. Kapranov, L. Langton, N. Perrimon, J.E. Sandler, K.H. Wan, A. Willingham, Y. Zhang, Y. Zou, J. Andrews, P.J. Bickel, S.E. Brenner, M.R. Brent, P. Cherbas, T.R. Gingeras, R.A. Hoskins, T.C. Kaufman, B. Oliver, and S.E. Celniker. The developmental transcriptome of Drosophila melanogaster. Nature, 471(7339):473-479, Mar 2011.

76. B. Guo, X. Huang, P. Zhang, L. Qi, Q. Liang, X. Zhang, J. Huang, B. Fang, W. Hou, J. Han, and H. Zhang. Genome-wide screen identifies signaling pathways that regulate autophagy during Caenorhabditis elegans development. EMBO Rep, 15(6):705-713, Jun 2014.

77. S. Han, S. Bahmanyar, P. Zhang, N. Grishin, K. Oegema, R. Crooke, M. Graham, K. Reue, J.E. Dixon, and J.M. Goodman. Nuclear envelope phosphatase 1-regulatory subunit 1 (formerly TMEM188) is the metazoan Spo7p ortholog and functions in the lipin activation pathway. J Biol Chem, 287(5):3123-3137, Jan 2012.

78. B.P. Head, M. Zulaika, S. Ryazantsev, and A.M. van der Bliek. A novel mitochondrial outer membrane protein, MOMA-1, that affects cristae morphology in Caenorhabditis elegans. Mol Biol Cell, 22(6):831-841, Mar 2011.

79. G. Heidary and M.E. Fortini. Identification and characterization of the Drosophila tau homolog. Mech Dev, 108(1-2):171-178, Oct 2001.

80. R. Herranz, J. Mateos, J.A. Mas, E. Garcia-Zaragoza, M. Cervera, and R. Marco. The coevolution of insect muscle TpnT and TpnI gene isoforms. Mol Biol Evol, 22(11):2231-2242, Nov 2005.

81. R.J. Hobson, Q. Liu, S. Watanabe, and E.M. Jorgensen. Complexin maintains vesicles in the primed state in C. elegans. Curr Biol, 21(2):106-113, Jan 2011.

82. S. Hokari, K. Miura, I. Koyama, M. Kobayashi, S. Komine, and T. Komoda. A restriction endonuclease assay for expression of human alpha-amylase isozymes. Clin Chim Acta, 322(1-2):113-116, Aug 2002.

83. L.Z. Holland, J.E. Carvalho, H. Escriva, V. Laudet, M. Schubert, S.M. Shimeld, and J.K. Yu. Evolution of bilaterian central nervous systems: a single origin? Evodevo, 4(1):27, 2013.

84. P.C. Hopkins. Neurodegeneration in a Drosophila model for the function of TMCC2, an amyloid protein precursor-interacting and apolipoprotein E-binding protein. PLoS One, 8(2):e55810, 2013.

85. B.E. Housden, A.Q. Fu, A. Krejci, F. Bernard, B. Fischer, S. Tavare, S. Russell, and S.J. Bray. Transcriptional dynamics elicited by a short pulse of notch activation involves feed-forward regulation by E(spl)/Hes genes. PLoS Genet, 9(1):e1003162, 2013.

86. S.M. Houten, S. Denis, H. Te Brinke, A. Jongejan, A.H. van Kampen, E.J. Bradley, F. Baas, R.C. Hennekam, D.S. Millington, S.P. Young, D.M. Frazier, M. Gucsavas-Calikoglu, and R.J. Wanders. Mitochondrial $\mathrm{NADP}(\mathrm{H})$ deficiency due to a mutation in NADK2 causes dienoyl-CoA reductase deficiency with hyperlysinemia. Hum Mol Genet, 23(18):5009-5016, Sep 2014.

87. D. Houzelstein, I.R. Goncalves, A.J. Fadden, S.S. Sidhu, D.N. Cooper, K. Drickamer, H. Leffler, and F. Poirier. Phylogenetic analysis of the vertebrate galectin family. Mol Biol Evol, 21(7):1177-1187, Jul 2004.

88. C.D. Hsiao, W.Y. Tsai, L.S. Horng, and H.J. Tsai. Molecular structure and developmental expression of three muscle-type troponin T genes in zebrafish. Dev Dyn, 227(2):266-279, Jun 2003. 
bioRxiv preprint doi: https://doi.org/10.1101/786723; this version posted September 30, 2019. The copyright holder for this preprint (which was not certified by peer review) is the author/funder, who has granted bioRxiv a license to display the preprint in perpetuity. It is made available under aCC-BY-NC-ND 4.0 International license.

89. S.Y. Hsu, Y.J. Chen, and P. Ouyang. Pnn and SR family proteins are differentially expressed in mouse central nervous system. Histochem Cell Biol, 135(4):361-373, Apr 2011.

90. S.Y. Hsu, Y.C. Cheng, H.Y. Shih, and P. Ouyang. Dissection of the role of Pinin in the development of zebrafish posterior pharyngeal cartilages. Histochem Cell Biol, 138(1):127-140, Jul 2012.

91. Z. Hu, D. Dandekar, P.J. O’Shaughnessy, K. De Gendt, G. Verhoeven, and M.F. Wilkinson. Androgeninduced Rhox homeobox genes modulate the expression of AR-regulated genes. Mol Endocrinol, 24(1):60-75, Jan 2010.

92. D. Huang, R. Meier, P.A. Todd, and L.M. Chou. Slow mitochondrial COI sequence evolution at the base of the metazoan tree and its implications for DNA barcoding. J Mol Evol, 66(2):167-174, Feb 2008.

93. S. Huang, S. Yuan, M. Dong, J. Su, C. Yu, Y. Shen, X. Xie, Y. Yu, X. Yu, S. Chen, S. Zhang, P. Pontarotti, and A. Xu. The phylogenetic analysis of tetraspanins projects the evolution of cell-cell interactions from unicellular to multicellular organisms. Genomics, 86(6):674-684, Dec 2005.

94. T. Hummel, K. Krukkert, J. Roos, G. Davis, and C. Klambt. Drosophila Futsch/22C10 is a MAP1B-like protein required for dendritic and axonal development. Neuron, 26(2):357-370, May 2000.

95. S. Huntwork and J.T. Littleton. A complexin fusion clamp regulates spontaneous neurotransmitter release and synaptic growth. Nat Neurosci, 10(10):1235-1237, Oct 2007.

96. N. Ichikawa, C. Ando, and M. Fumino. Caenorhabditis elegans MAI-1 protein, which is similar to mitochondrial ATPase inhibitor (IF1), can inhibit yeast F0F1-ATPase but cannot be transported to yeast mitochondria. J Bioenerg Biomembr, 38(2):93-99, Apr 2006.

97. S. Ihara, E.J. Hagedorn, M.A. Morrissey, Q. Chi, F. Motegi, J.M. Kramer, and D.R. Sherwood. Basement membrane sliding and targeted adhesion remodels tissue boundaries during uterine-vulval attachment in Caenorhabditis elegans. Nat Cell Biol, 13(6):641-651, Jun 2011.

98. K. Iijima-Ando, M. Sekiya, A. Maruko-Otake, Y. Ohtake, E. Suzuki, B. Lu, and K.M. Iijima. Loss of axonal mitochondria promotes tau-mediated neurodegeneration and Alzheimer's disease-related tau phosphorylation via PAR-1. PLoS Genet, 8(8):e1002918, 2012.

99. J.W. Jang, W.Y. Lee, J.H. Lee, S.H. Moon, C.H. Kim, and H.M. Chung. A novel Fbxo25 acts as an E3 ligase for destructing cardiac specific transcription factors. Biochem Biophys Res Commun, 410(2):183-188, Jul 2011.

100. G. Jekely. Global view of the evolution and diversity of metazoan neuropeptide signaling. Proc Natl Acad Sci U S A, 110(21):8702-8707, May 2013.

101. L. Jiang and S.T. Crews. Dysfusion transcriptional control of Drosophila tracheal migration, adhesion, and fusion. Mol Cell Biol, 26(17):6547-6556, Sep 2006.

102. L. Jiang, J.C. Pearson, and S.T. Crews. Diverse modes of Drosophila tracheal fusion cell transcriptional regulation. Mech Dev, 127(5-6):265-280, May 2010.

103. S. Jin, L. Pan, Z. Liu, Q. Wang, Z. Xu, and Y.Q. Zhang. Drosophila Tubulin-specific chaperone E functions at neuromuscular synapses and is required for microtubule network formation. Development, 136(9):1571-1581, May 2009.

104. J.H. Joo, T.J. Taxter, G.C. Munguba, Y.H. Kim, K. Dhaduvai, N.W. Dunn, W.J. Degan, S.P. Oh, and S.P. Sugrue. Pinin modulates expression of an intestinal homeobox gene, Cdx2, and plays an essential role for small intestinal morphogenesis. Dev Biol, 345(2):191-203, Sep 2010.

105. T. Kanome, N. Itoh, F. Ishikawa, K. Mori, J.R. Kim-Kaneyama, K. Nose, and M. Shibanuma. Characterization of Jumping translocation breakpoint (JTB) gene product isolated as a TGF-beta1inducible clone involved in regulation of mitochondrial function, cell growth and cell death. Oncogene, 26(41):5991-6001, Sep 2007.

106. E.D. Karousis and D.C. Sideris. A subtle alternative splicing event gives rise to a widely expressed human RNase k isoform. PLoS One, 9(5):e96557, 2014. 
bioRxiv preprint doi: https://doi.org/10.1101/786723; this version posted September 30, 2019. The copyright holder for this preprint (which was not certified by peer review) is the author/funder, who has granted bioRxiv a license to display the preprint in perpetuity. It is made available under aCC-BY-NC-ND 4.0 International license.

107. J. Kim, S. Lee, M. Hwang, S. Ko, C. Min, and J. Kim-Ha. Bunched specifically regulates alpha/beta mushroom body neuronal cell proliferation during metamorphosis. Neuroscience, 161(1):46-52, Jun 2009.

108. J.D. Kim, H. Kang, B. Larrivee, M.Y. Lee, M. Mettlen, S.L. Schmid, B.L. Roman, Y. Qyang, A. Eichmann, and S.W. Jin. Context-dependent proangiogenic function of bone morphogenetic protein signaling is mediated by disabled homolog 2. Dev Cell, 23(2):441-448, Aug 2012.

109. Y.I. Kim, S. Lee, S.H. Jung, H.T. Kim, J.H. Choi, M.S. Lee, K.H. You, S.Y. Yeo, K.W. Yoo, S. Kwak, J.N. Lee, R. Park, S.K. Choe, and C.H. Kim. Establishment of a bone-specific col10a1:GFP transgenic zebrafish. Mol Cells, 36(2):145-150, Aug 2013.

110. J. Kitano, Y. Yamazaki, K. Kimura, T. Masukado, Y. Nakajima, and S. Nakanishi. Tamalin is a scaffold protein that interacts with multiple neuronal proteins in distinct modes of protein-protein association. J Biol Chem, 278(17):14762-14768, Apr 2003.

111. T. Komori. Regulation of bone development and extracellular matrix protein genes by RUNX2. Cell Tissue Res, 339(1):189-195, Jan 2010.

112. M. Kostrouchova, M. Krause, Z. Kostrouch, and J.E. Rall. Nuclear hormone receptor CHR3 is a critical regulator of all four larval molts of the nematode Caenorhabditis elegans. Proc Natl Acad Sci U S A, 98(13):7360-7365, Jun 2001.

113. N.A. Kouns, J. Nakielna, F. Behensky, M.W. Krause, Z. Kostrouch, and M. Kostrouchova. NHR-23 dependent collagen and hedgehog-related genes required for molting. Biochem Biophys Res Commun, 413(4):515-520, Oct 2011.

114. B.C. Kraemer and G.D. Schellenberg. SUT-1 enables tau-induced neurotoxicity in C. elegans. Hum Mol Genet, 16(16):1959-1971, Aug 2007.

115. A.J. Krol, D. Roellig, M.L. Dequeant, O. Tassy, E. Glynn, G. Hattem, A. Mushegian, A.C. Oates, and O. Pourquie. Evolutionary plasticity of segmentation clock networks. Development, 138(13):2783-2792, Jul 2011.

116. R. Labrecque, C. Vigneault, P. Blondin, and M.A. Sirard. Gene expression analysis of bovine oocytes at optimal coasting time combined with GnRH antagonist during the no-FSH period. Theriogenology, 81(8):1092-1100, May 2014.

117. M.C. Lagerstrom and H.B. Schioth. Structural diversity of G protein-coupled receptors and significance for drug discovery. Nat Rev Drug Discov, 7(4):339-357, Apr 2008.

118. M. Lagha, J.P. Bothma, E. Esposito, S. Ng, L. Stefanik, C. Tsui, J. Johnston, K. Chen, D.S. Gilmour, J. Zeitlinger, and M.S. Levine. Paused Pol II coordinates tissue morphogenesis in the Drosophila embryo. Cell, 153(5):976-987, May 2013.

119. D.C. Lanza, D.M. Trindade, E.M. Assmann, and J. Kobarg. Over-expression of GFP-FEZ1 causes generation of multi-lobulated nuclei mediated by microtubules in HEK293 cells. Exp Cell Res, 314(10):2028-2039, Jun 2008.

120. M. Larhammar, K. Patra, M. Blunder, L. Emilsson, C. Peuckert, E. Arvidsson, D. Ronnlund, J. Preobraschenski, C. Birgner, C. Limbach, J. Widengren, H. Blom, R. Jahn, A. Wallen-Mackenzie, and K. Kullander. SLC10A4 is a vesicular amine-associated transporter modulating dopamine homeostasis. Biol Psychiatry, 77(6):526-536, Mar 2015.

121. K.S. Lee and B. Lu. The myriad roles of Miro in the nervous system: axonal transport of mitochondria and beyond. Front Cell Neurosci, 8:330, 2014.

122. R. Levayer and E. Moreno. Mechanisms of cell competition: themes and variations. J Cell Biol, 200(6):689-698, Mar 2013.

123. M.C. Levendusky, J. Basle, S. Chang, N.V. Mandalaywala, J.M. Voigt, and R.E. Dearborn, Jr. Expression and regulation of vitamin D3 upregulated protein 1 (VDUP1) is conserved in mammalian and insect brain. J Comp Neurol, 517(5):581-600, Dec 2009.

124. M. Levin, T. Hashimshony, F. Wagner, and I. Yanai. Developmental milestones punctuate gene expression in the Caenorhabditis embryo. Dev Cell, 22(5):1101-1108, May 2012. 
bioRxiv preprint doi: https://doi.org/10.1101/786723; this version posted September 30, 2019. The copyright holder for this preprint (which was not certified by peer review) is the author/funder, who has granted bioRxiv a license to display the preprint in perpetuity. It is made available under aCC-BY-NC-ND 4.0 International license.

125. C.Y. Lin, H.C. Lee, H.C. Chen, C.C. Hsieh, and H.J. Tsai. Normal function of Myf5 during gastrulation is required for pharyngeal arch cartilage development in zebrafish embryos. Zebrafish, 10(4):486-499, Dec 2013.

126. J.X. Liu, B. Hu, Y. Wang, J.F. Gui, and W. Xiao. Zebrafish eaf1 and eaf2/u19 mediate effective convergence and extension movements through the maintenance of wnt11 and wnt5 expression. $J$ Biol Chem, 284(24):16679-16692, Jun 2009.

127. J.X. Liu, D. Zhang, X. Xie, G. Ouyang, X. Liu, Y. Sun, and W. Xiao. Eaf1 and Eaf2 negatively regulate canonical Wnt/beta-catenin signaling. Development, 140(5):1067-1078, Mar 2013.

128. L. Liu, D. Feng, G. Chen, M. Chen, Q. Zheng, P. Song, Q. Ma, C. Zhu, R. Wang, W. Qi, L. Huang, P. Xue, B. Li, X. Wang, H. Jin, J. Wang, F. Yang, P. Liu, Y. Zhu, S. Sui, and Q. Chen. Mitochondrial outer-membrane protein FUNDC1 mediates hypoxia-induced mitophagy in mammalian cells. Nat Cell Biol, 14(2):177-185, Feb 2012.

129. F.N. Lolo, S. Casas-Tinto, and E. Moreno. Cell competition time line: winners kill losers, which are extruded and engulfed by hemocytes. Cell Rep, 2(3):526-539, Sep 2012.

130. Y. Lv, K. Zhang, and H. Gao. Paip1, an effective stimulator of translation initiation, is targeted by WWP2 for ubiquitination and degradation. Mol Cell Biol, 34(24):4513-4522, Dec 2014.

131. X. Ma and J.X. Liu. Eafs control erythroid cell fate by regulating c-myb expression through Wnt signaling. PLoS One, 8(5):e64576, 2013.

132. M.C. Marin, J.R. Rodriguez, and A. Ferrus. Transcription of Drosophila troponin i gene is regulated by two conserved, functionally identical, synergistic elements. Mol Biol Cell, 15(3):1185-1196, Mar 2004.

133. J.A. Martin, Z. Hu, K.M. Fenz, J. Fernandez, and J.S. Dittman. Complexin has opposite effects on two modes of synaptic vesicle fusion. Curr Biol, 21(2):97-105, Jan 2011.

134. P.M. Martin, M. Carnaud, G. Garcia del Cano, M. Irondelle, T. Irinopoulou, J.A. Girault, B. Dargent, and L. Goutebroze. Schwannomin-interacting protein-1 isoform IQCJ-SCHIP-1 is a late component of nodes of Ranvier and axon initial segments. J Neurosci, 28(24):6111-6117, Jun 2008.

135. N. Martinek, J. Shahab, M. Saathoff, and M. Ringuette. Haemocyte-derived SPARC is required for collagen-IV-dependent stability of basal laminae in Drosophila embryos. J Cell Sci, 121(Pt 10):1671-1680, May 2008.

136. S.J. Marygold, J. Roote, G. Reuter, A. Lambertsson, M. Ashburner, G.H. Millburn, P.M. Harrison, Z. Yu, N. Kenmochi, T.C. Kaufman, S.J. Leevers, and K.R. Cook. The ribosomal protein genes and Minute loci of Drosophila melanogaster. Genome Biol, 8(10):R216, 2007.

137. J.A. Mas, E. Garcia-Zaragoza, and M. Cervera. Two functionally identical modular enhancers in Drosophila troponin T gene establish the correct protein levels in different muscle types. Mol Biol Cell, 15(4):1931-1945, Apr 2004.

138. C.A. Matthews, J.E. Shaw, J.A. Hooper, I.G. Young, M.F. Crouch, and H.D. Campbell. Expression and evolution of the mammalian brain gene Ttyh1. J Neurochem, 100(3):693-707, Feb 2007.

139. A.D. Maturana, T. Fujita, and S. Kuroda. Functions of fasciculation and elongation protein zeta-1 (FEZ1) in the brain. Scientific WorldJournal, 10:1646-1654, 2010.

140. M. Mazzocco, M. Maffei, A. Egeo, A. Vergano, P. Arrigo, R. Di Lisi, F. Ghiotto, and P. Scartezzini. The identification of a novel human homologue of the SH3 binding glutamic acid-rich (SH3BGR) gene establishes a new family of highly conserved small proteins related to Thioredoxin Superfamily. Gene, 291(1-2):233-239, May 2002.

141. S. McNabb, S. Greig, and T. Davis. The alcohol dehydrogenase gene is nested in the outspread locus of Drosophila melanogaster. Genetics, 143(2):897-911, Jun 1996.

142. T.J. Melia, Jr. Putting the clamps on membrane fusion: how complexin sets the stage for calciummediated exocytosis. FEBS Lett, 581(11):2131-2139, May 2007. 
bioRxiv preprint doi: https://doi.org/10.1101/786723; this version posted September 30, 2019. The copyright holder for this preprint (which was not certified by peer review) is the author/funder, who has granted bioRxiv a license to display the preprint in perpetuity. It is made available under aCC-BY-NC-ND 4.0 International license.

143. A.M. Michelson, S.M. Abmayr, M. Bate, A.M. Arias, and T. Maniatis. Expression of a MyoD family member prefigures muscle pattern in Drosophila embryos. Genes Dev, 4(12A):2086-2097, Dec 1990.

144. S. Miehe, A. Bieberstein, I. Arnould, O. Ihdene, H. Rutten, and C. Strubing. The phospholipid-binding protein SESTD1 is a novel regulator of the transient receptor potential channels TRPC4 and TRPC5. J Biol Chem, 285(16):12426-12434, Apr 2010.

145. O. Mirabeau and J.S. Joly. Molecular evolution of peptidergic signaling systems in bilaterians. Proc Natl Acad Sci U S A, 110(22):E2028-37, May 2013.

146. M. Misiak, S. Singh, S. Drewlo, C. Beyer, and S. Arnold. Brain region-specific vulnerability of astrocytes in response to 3-nitropropionic acid is mediated by cytochrome c oxidase isoform expression. Cell Tissue Res, 341(1):83-93, Jul 2010.

147. R.W. Mitchell and G.M. Hatch. Fatty acid transport into the brain: of fatty acid fables and lipid tails. Prostaglandins Leukot Essent Fatty Acids, 85(5):293-302, Nov 2011.

148. H. Moribe and E. Mekada. Co-occurrence of tetraspanin and ROS generators: Conservation in protein cross-linking and other developmental processes. Worm, 2(2):e23415, Apr 2013.

149. H. Moribe, J. Yochem, H. Yamada, Y. Tabuse, T. Fujimoto, and E. Mekada. Tetraspanin protein (TSP-15) is required for epidermal integrity in Caenorhabditis elegans. J Cell Sci, 117(Pt 22):5209-5220, Oct 2004 .

150. X. Mou, D.M. Duncan, E.H. Baehrecke, and I. Duncan. Control of target gene specificity during metamorphosis by the steroid response gene E93. Proc Natl Acad Sci U S A, 109(8):2949-2954, Feb 2012.

151. V.S. Moulle, C. Cansell, S. Luquet, and C. Cruciani-Guglielmacci. The multiple roles of fatty acid handling proteins in brain. Front Physiol, 3:385, 2012.

152. C.D. Myers, P.Y. Goh, T.S. Allen, E.A. Bucher, and T. Bogaert. Developmental genetic analysis of troponin T mutations in striated and nonstriated muscle cells of Caenorhabditis elegans. J Cell Biol, 132(6):1061-1077, Mar 1996.

153. A. Nagao, T. Suzuki, T. Katoh, Y. Sakaguchi, and T. Suzuki. Biogenesis of glutaminyl-mt tRNAGln in human mitochondria. Proc Natl Acad Sci U S A, 106(38):16209-16214, Sep 2009.

154. B. Naimi, A. Harrison, M. Cummins, U. Nongthomba, S. Clark, I. Canal, A. Ferrus, and J.C. Sparrow. A tropomyosin-2 mutation suppresses a troponin I myopathy in Drosophila. Mol Biol Cell, 12(5):1529-1539, May 2001.

155. M. Nakamura, D. Baldwin, S. Hannaford, J. Palka, and C. Montell. Defective proboscis extension response (DPR), a member of the Ig superfamily required for the gustatory response to salt. $J$ Neurosci, 22(9):3463-3472, May 2002.

156. V.H. Nguyen, B. Schmid, J. Trout, S.A. Connors, M. Ekker, and M.C. Mullins. Ventral and lateral regions of the zebrafish gastrula, including the neural crest progenitors, are established by a bmp2b/swirl pathway of genes. Dev Biol, 199(1):93-110, Jul 1998.

157. U. Nongthomba, M. Ansari, D. Thimmaiya, M. Stark, and J. Sparrow. Aberrant splicing of an alternative exon in the Drosophila troponin- $\mathrm{T}$ gene affects flight muscle development. Genetics, 177(1):295-306, Sep 2007.

158. T.W. O'Brien. Evolution of a protein-rich mitochondrial ribosome: implications for human genetic disease. Gene, 286(1):73-79, Mar 2002.

159. K. Ohara, A. Enomoto, T. Kato, T. Hashimoto, M. Isotani-Sakakibara, N. Asai, M. Ishida-Takagishi, L. Weng, M. Nakayama, T. Watanabe, K. Kato, K. Kaibuchi, Y. Murakumo, Y. Hirooka, H. Goto, and M. Takahashi. Involvement of Girdin in the determination of cell polarity during cell migration. PLoS One, 7(5):e36681, 2012.

160. M. Okumura, A.M. Katsuyama, H. Shibata, and M. Maki. VPS37 isoforms differentially modulate the ternary complex formation of ALIX, ALG-2, and ESCRT-i. Biosci Biotechnol Biochem, 77(8):17151721, 2013. 
bioRxiv preprint doi: https://doi.org/10.1101/786723; this version posted September 30, 2019. The copyright holder for this preprint (which was not certified by peer review) is the author/funder, who has granted bioRxiv a license to display the preprint in perpetuity. It is made available under aCC-BY-NC-ND 4.0 International license.

161. G. Oliver, B. Sosa-Pineda, S. Geisendorf, E.P. Spana, C.Q. Doe, and P. Gruss. Prox 1, a prosperorelated homeobox gene expressed during mouse development. Mech Dev, 44(1):3-16, Nov 1993.

162. H. Ota, T. Hikita, T. Nishioka, M. Matsumoto, J. Ito, N. Asai, A. Enomoto, M. Takahashi, K. Kaibuchi, K. Sobue, and K. Sawamoto. Proteomic analysis of Girdin-interacting proteins in migrating new neurons in the postnatal mouse brain. Biochem Biophys Res Commun, 442(1-2):16-21, Dec 2013.

163. H. Parikh, E. Nilsson, C. Ling, P. Poulsen, P. Almgren, H. Nittby, K.F. Eriksson, A. Vaag, and L.C. Groop. Molecular correlates for maximal oxygen uptake and type 1 fibers. Am J Physiol Endocrinol Metab, 294(6):E1152-9, Jun 2008.

164. S.E. Patterson, N.C. Bird, and S.H. Devoto. BMP regulation of myogenesis in zebrafish. Dev Dyn, 239(3):806-817, Mar 2010.

165. M. Pejznochova, M. Tesarova, H. Hansikova, M. Magner, T. Honzik, K. Vinsova, Z. Hajkova, V. Havlickova, and J. Zeman. Mitochondrial DNA content and expression of genes involved in mtDNA transcription, regulation and maintenance during human fetal development. Mitochondrion, 10(4):321-329, Jun 2010.

166. C. Picco, J. Scholz-Starke, A. Naso, V. Preger, F. Sparla, P. Trost, and A. Carpaneto. How are cytochrome b561 electron currents controlled by membrane voltage and substrate availability? Antioxid Redox Signal, 21(3):384-391, Jul 2014.

167. S.N. Popova and I. Alafuzoff. Distribution of SLC10A4, a synaptic vesicle protein in the human brain, and the association of this protein with Alzheimer's disease-related neuronal degeneration. $J$ Alzheimers Dis, 37(3):603-610, 2013.

168. M. Portela, S. Casas-Tinto, C. Rhiner, J.M. Lopez-Gay, O. Dominguez, D. Soldini, and E. Moreno. Drosophila SPARC is a self-protective signal expressed by loser cells during cell competition. Dev Cell, 19(4):562-573, Oct 2010.

169. J.A. Powell-Coffman, J. Knight, and W.B. Wood. Onset of C. elegans gastrulation is blocked by inhibition of embryonic transcription with an RNA polymerase antisense RNA. Dev Biol, 178(2):472483, Sep 1996.

170. G. Ranganayakulu, R.A. Schulz, and E.N. Olson. Wingless signaling induces nautilus expression in the ventral mesoderm of the Drosophila embryo. Dev Biol, 176(1):143-148, May 1996.

171. E. Reinstein, K. Orvin, E. Tayeb-Fligelman, H. Stiebel-Kalish, S. Tzur, A.L. Pimienta, L. Bazak, T. Bengal, L. Cohen, D.D. Gaton, C. Bormans, M. Landau, R. Kornowski, M. Shohat, and D.M. Behar. Mutations in TAX1BP3 cause dilated cardiomyopathy with septo-optic dysplasia. Hum Mutat, 36(4):439-442, Apr 2015.

172. M. Renaud, V. Praz, E. Vieu, L. Florens, M.P. Washburn, P. l'Hote, and N. Hernandez. Gene duplication and neofunctionalization: POLR3G and POLR3GL. Genome Res, 24(1):37-51, Jan 2014.

173. C. Rhiner, J.M. Lopez-Gay, D. Soldini, S. Casas-Tinto, F.A. Martin, L. Lombardia, and E. Moreno. Flower forms an extracellular code that reveals the fitness of a cell to its neighbors in Drosophila. Dev Cell, 18(6):985-998, Jun 2010.

174. C.E. Rocheleau, A. Ronnlund, S. Tuck, and M.V. Sundaram. Caenorhabditis elegans CNK-1 promotes Raf activation but is not essential for Ras/Raf signaling. Proc Natl Acad Sci U S A, 102(33):1175711762, Aug 2005.

175. J. Roos, T. Hummel, N. Ng, C. Klambt, and G.W. Davis. Drosophila Futsch regulates synaptic microtubule organization and is necessary for synaptic growth. Neuron, 26(2):371-382, May 2000.

176. G.E. Roth, M.S. Gierl, L. Vollborn, M. Meise, R. Lintermann, and G. Korge. The Drosophila gene Start1: a putative cholesterol transporter and key regulator of ecdysteroid synthesis. Proc Natl Acad Sci U S A, 101(6):1601-1606, Feb 2004.

177. A.F. Ruaud, G. Lam, and C.S. Thummel. The Drosophila nuclear receptors DHR3 and betaFTZ-F1 control overlapping developmental responses in late embryos. Development, 137(1):123-131, Jan 2010. 
bioRxiv preprint doi: https://doi.org/10.1101/786723; this version posted September 30, 2019. The copyright holder for this preprint (which was not certified by peer review) is the author/funder, who has granted bioRxiv a license to display the preprint in perpetuity. It is made available under aCC-BY-NC-ND 4.0 International license.

178. E.I. Rugarli, E. Di Schiavi, M.A. Hilliard, S. Arbucci, C. Ghezzi, A. Facciolli, G. Coppola, A. Ballabio, and P. Bazzicalupo. The Kallmann syndrome gene homolog in C. elegans is involved in epidermal morphogenesis and neurite branching. Development, 129(5):1283-1294, Mar 2002.

179. B.M. Scheel and B. Hausdorf. Dynamic evolution of mitochondrial ribosomal proteins in Holozoa. Mol Phylogenet Evol, 76:67-74, Jul 2014.

180. J.E. Schwarzbauer and C.S. Spencer. The Caenorhabditis elegans homologue of the extracellular calcium binding protein SPARC/osteonectin affects nematode body morphology and mobility. Mol Biol Cell, 4(9):941-952, Sep 1993.

181. J. Schwendinger-Schreck, Y. Kang, and S.A. Holley. Modeling the zebrafish segmentation clock's gene regulatory network constrained by expression data suggests evolutionary transitions between oscillating and nonoscillating transcription. Genetics, 197(2):725-738, Jun 2014.

182. D.I. Shah, N. Takahashi-Makise, J.D. Cooney, L. Li, I.J. Schultz, E.L. Pierce, A. Narla, A. Seguin, S.M. Hattangadi, A.E. Medlock, N.B. Langer, T.A. Dailey, S.N. Hurst, D. Faccenda, J.M. Wiwczar, S.K. Heggers, G. Vogin, W. Chen, C. Chen, D.R. Campagna, C. Brugnara, Y. Zhou, B.L. Ebert, N.N. Danial, M.D. Fleming, D.M. Ward, M. Campanella, H.A. Dailey, J. Kaplan, and B.H. Paw. Mitochondrial Atpif1 regulates haem synthesis in developing erythroblasts. Nature, 491(7425):608-612, Nov 2012.

183. T.L. Shearer, M.J. Van Oppen, S.L. Romano, and G. Worheide. Slow mitochondrial DNA sequence evolution in the Anthozoa (Cnidaria). Mol Ecol, 11(12):2475-2487, Dec 2002.

184. T. Siegmund and M. Lehmann. The Drosophila Pipsqueak protein defines a new family of helix-turnhelix DNA-binding proteins. Dev Genes Evol, 212(3):152-157, Apr 2002.

185. J.S. Simske. Claudins reign: The claudin/EMP/PMP22/gamma channel protein family in C. elegans. Tissue Barriers, 1(3):e25502, Jul 2013.

186. J.S. Simske, M. Koppen, P. Sims, J. Hodgkin, A. Yonkof, and J. Hardin. The cell junction protein VAB-9 regulates adhesion and epidermal morphology in C. elegans. Nat Cell Biol, 5(7):619-625, Jul 2003.

187. E.R. Smith, B. Winter, J.C. Eissenberg, and A. Shilatifard. Regulation of the transcriptional activity of poised RNA polymerase II by the elongation factor ELL. Proc Natl Acad Sci U S A, 105(25):8575-8579, Jun 2008.

188. P. Smits, J.A. Smeitink, L.P. van den Heuvel, M.A. Huynen, and T.J. Ettema. Reconstructing the evolution of the mitochondrial ribosomal proteome. Nucleic Acids Res, 35(14):4686-4703, 2007.

189. J.K. Song, R. Kannan, G. Merdes, J. Singh, M. Mlodzik, and E. Giniger. Disabled is a bona fide component of the Abl signaling network. Development, 137(21):3719-3727, Nov 2010.

190. P.R. Steinmetz, J.E. Kraus, C. Larroux, J.U. Hammel, A. Amon-Hassenzahl, E. Houliston, G. Worheide, M. Nickel, B.M. Degnan, and U. Technau. Independent evolution of striated muscles in cnidarians and bilaterians. Nature, 487(7406):231-234, Jul 2012.

191. A.D. Stump, M. Dillon-White, and S. Gu. Molecular evolution of the moonlighting protein SMN in metazoans. Comp Biochem Physiol Part D Genomics Proteomics, 8(3):220-230, Sep 2013.

192. V. Subramaniam, P. Golik, D.G. Murdock, S. Levy, K.W. Kerstann, P.E. Coskun, G.A. Melkonian, and D.C. Wallace. MITOCHIP assessment of differential gene expression in the skeletal muscle of Ant1 knockout mice: coordinate regulation of OXPHOS, antioxidant, and apoptotic genes. Biochim Biophys Acta, 1777(7-8):666-675, Jul 2008.

193. H. Sun, T. Tsunenari, K.W. Yau, and J. Nathans. The vitelliform macular dystrophy protein defines a new family of chloride channels. Proc Natl Acad Sci U S A, 99(6):4008-4013, Mar 2002.

194. Y. Takashima, S. Kitaoka, T. Bando, and H. Kagawa. Expression profiles and unc-27 mutation rescue of the striated muscle type troponin I isoform-3 in Caenorhabditis elegans. Genes Genet Syst, 87(4):243-251, 2012.

195. S.J. Tapscott. The circuitry of a master switch: Myod and the regulation of skeletal muscle gene transcription. Development, 132(12):2685-2695, Jun 2005. 
bioRxiv preprint doi: https://doi.org/10.1101/786723; this version posted September 30, 2019. The copyright holder for this preprint (which was not certified by peer review) is the author/funder, who has granted bioRxiv a license to display the preprint in perpetuity. It is made available under aCC-BY-NC-ND 4.0 International license.

196. L. Teixeira. Whole-genome expression profile analysis of Drosophila melanogaster immune responses. Brief Funct Genomics, 11(5):375-386, Sep 2012.

197. K. Tessmar-Raible, F. Raible, F. Christodoulou, K. Guy, M. Rembold, H. Hausen, and D. Arendt. Conserved sensory-neurosecretory cell types in annelid and fish forebrain: insights into hypothalamus evolution. Cell, 129(7):1389-1400, Jun 2007.

198. M. Therrien, A.M. Wong, and G.M. Rubin. CNK, a RAF-binding multidomain protein required for RAS signaling. Cell, 95(3):343-353, Oct 1998.

199. H. Toda, H. Mochizuki, R. Flores, 3rd, R. Josowitz, T.B. Krasieva, V.J. Lamorte, E. Suzuki, J.G. Gindhart, K. Furukubo-Tokunaga, and T. Tomoda. UNC-51/ATG1 kinase regulates axonal transport by mediating motor-cargo assembly. Genes Dev, 22(23):3292-3307, Dec 2008.

200. E. Torres Nunez, C. Sobrino, P.J. Neale, R.M. Ceinos, S. Du, and J. Rotllant. Molecular response to ultraviolet radiation exposure in fish embryos: implications for survival and morphological development. Photochem Photobiol, 88(3):701-707, May 2012.

201. M. van Spronsen, M. Mikhaylova, J. Lipka, M.A. Schlager, D.J. van den Heuvel, M. Kuijpers, P.S. Wulf, N. Keijzer, J. Demmers, L.C. Kapitein, D. Jaarsma, H.C. Gerritsen, A. Akhmanova, and C.C. Hoogenraad. TRAK/Milton motor-adaptor proteins steer mitochondrial trafficking to axons and dendrites. Neuron, 77(3):485-502, Feb 2013.

202. D. Van Vactor, D.P. Wall, and K.G. Johnson. Heparan sulfate proteoglycans and the emergence of neuronal connectivity. Curr Opin Neurobiol, 16(1):40-51, Feb 2006.

203. L. Wang, Y. Sun, M. Jiang, and X. Zheng. Integrative decomposition procedure and Kappa statistics for the distinguished single molecular network construction and analysis. J Biomed Biotechnol, 2009:726728, 2009.

204. P. Wang, P.J. Lou, S. Leu, and P. Ouyang. Modulation of alternative pre-mRNA splicing in vivo by pinin. Biochem Biophys Res Commun, 294(2):448-455, Jun 2002.

205. Y. Wang, N. Kaneko, N. Asai, A. Enomoto, M. Isotani-Sakakibara, T. Kato, M. Asai, Y. Murakumo, H. Ota, T. Hikita, T. Namba, K. Kuroda, K. Kaibuchi, G.L. Ming, H. Song, K. Sawamoto, and M. Takahashi. Girdin is an intrinsic regulator of neuroblast chain migration in the rostral migratory stream of the postnatal brain. J Neurosci, 31(22):8109-8122, Jun 2011.

206. Z. Wang and R.G. Roeder. Three human RNA polymerase III-specific subunits form a subcomplex with a selective function in specific transcription initiation. Genes Dev, 11(10):1315-1326, May 1997.

207. K.J. Webb, M. Coolen, C.J. Gloeckner, C. Stigloher, B. Bahn, S. Topp, M. Ueffing, and L. Bally-Cuif. The enhancer of split transcription factor Her8a is a novel dimerisation partner for Her3 that controls anterior hindbrain neurogenesis in zebrafish. BMC Dev Biol, 11:27, 2011.

208. Q. Wei, Y. Rong, and B.M. Paterson. Stereotypic founder cell patterning and embryonic muscle formation in Drosophila require nautilus (MyoD) gene function. Proc Natl Acad Sci U S A, 104(13):54615466, Mar 2007.

209. R.C. Wong, S. Pollan, H. Fong, A. Ibrahim, E.L. Smith, M. Ho, A.L. Laslett, and P.J. Donovan. A novel role for an RNA polymerase III subunit POLR3G in regulating pluripotency in human embryonic stem cells. Stem Cells, 29(10):1517-1527, Oct 2011.

210. H.P. Wu, S.Y. Hsu, W.A. Wu, J.W. Hu, and P. Ouyang. Transgenic mice expressing mutant Pinin exhibit muscular dystrophy, nebulin deficiency and elevated expression of slow-type muscle fiber genes. Biochem Biophys Res Commun, 443(1):313-320, Jan 2014.

211. W. Wu, W. Tian, Z. Hu, G. Chen, L. Huang, W. Li, X. Zhang, P. Xue, C. Zhou, L. Liu, Y. Zhu, X. Zhang, L. Li, L. Zhang, S. Sui, B. Zhao, and D. Feng. ULK1 translocates to mitochondria and phosphorylates FUNDC1 to regulate mitophagy. EMBO Rep, 15(5):566-575, May 2014.

212. D. Yamazaki, Y. Tabara, S. Kita, H. Hanada, S. Komazaki, D. Naitou, A. Mishima, M. Nishi, H. Yamamura, S. Yamamoto, S. Kakizawa, H. Miyachi, S. Yamamoto, T. Miyata, Y. Kawano, K. Kamide, T. Ogihara, A. Hata, S. Umemura, M. Soma, N. Takahashi, Y. Imaizumi, T. Miki, T. Iwamoto, and H. Takeshima. TRIC-A channels in vascular smooth muscle contribute to blood pressure maintenance. Cell Metab, 14(2):231-241, Aug 2011. 
bioRxiv preprint doi: https://doi.org/10.1101/786723; this version posted September 30, 2019. The copyright holder for this preprint (which was not certified by peer review) is the author/funder, who has granted bioRxiv a license to display the preprint in perpetuity. It is made available under aCC-BY-NC-ND 4.0 International license.

213. T. Yamazaki, N. Fujiwara, H. Yukinaga, M. Ebisuya, T. Shiki, T. Kurihara, N. Kioka, T. Kambe, M. Nagao, E. Nishida, and S. Masuda. The closely related RNA helicases, UAP56 and URH49, preferentially form distinct mRNA export machineries and coordinately regulate mitotic progression. Mol Biol Cell, 21(16):2953-2965, Aug 2010.

214. X. Yang and B.N. Cheyette. SEC14 and spectrin domains 1 (Sestd1) and Dapper antagonist of catenin 1 (Dact1) scaffold proteins cooperatively regulate the Van Gogh-like 2 (Vangl2) four-pass transmembrane protein and planar cell polarity $(\mathrm{PCP})$ pathway during embryonic development in mice. J Biol Chem, 288(28):20111-20120, Jul 2013.

215. U. Yavuzer, G.C. Smith, T. Bliss, D. Werner, and S.P. Jackson. DNA end-independent activation of DNA-PK mediated via association with the DNA-binding protein C1D. Genes Dev, 12(14):2188-2199, Jul 1998.

216. J.H. Yim, Y.J. Kim, J.H. Ko, Y.E. Cho, S.M. Kim, J.Y. Kim, S. Lee, and J.H. Park. The putative tumor suppressor gene GLTSCR2 induces PTEN-modulated cell death. Cell Death Differ, 14(11):18721879, Nov 2007.

217. J.C. Yoon, A.J. Ling, M. Isik, D.Y. Lee, M.J. Steinbaugh, L.M. Sack, A.N. Boduch, T.K. Blackwell, D.A. Sinclair, and S.J. Elledge. GLTSCR2/PICT1 links mitochondrial stress and Myc signaling. Proc Natl Acad Sci U S A, 111(10):3781-3786, Mar 2014.

218. T.Y. Yoon, X. Lu, J. Diao, S.M. Lee, T. Ha, and Y.K. Shin. Complexin and Ca2+ stimulate SNARE-mediated membrane fusion. Nat Struct Mol Biol, 15(7):707-713, Jul 2008.

219. H. Yu, R.F. Pretot, T.R. Burglin, and P.W. Sternberg. Distinct roles of transcription factors EGL-46 and DAF-19 in specifying the functionality of a polycystin-expressing sensory neuron necessary for $C$. elegans male vulva location behavior. Development, 130(21):5217-5227, Nov 2003.

220. E. Zacharioudaki, S.S. Magadi, and C. Delidakis. bHLH-O proteins are crucial for Drosophila neuroblast self-renewal and mediate Notch-induced overproliferation. Development, 139(7):1258-1269, Apr 2012.

221. C. Zhang, Y.S. Kho, Z. Wang, Y.T. Chiang, G.K. Ng, P.C. Shaw, Y. Wang, and R.Z. Qi. Transmembrane and coiled-coil domain family 1 is a novel protein of the endoplasmic reticulum. PLoS One, 9(1):e85206, 2014.

222. P. Zhang, X. Ma, E. Song, W. Chen, H. Pang, D. Ni, Y. Gao, Y. Fan, Q. Ding, Y. Zhang, and $\mathrm{X}$. Zhang. Tubulin cofactor a functions as a novel positive regulator of ccRCC progression, invasion and metastasis. Int $J$ Cancer, 133(12):2801-2811, Dec 2013.

223. X. Zhang, C.C. Milton, C.L. Poon, W. Hong, and K.F. Harvey. Wbp2 cooperates with Yorkie to drive tissue growth downstream of the Salvador-Warts-Hippo pathway. Cell Death Differ, 18(8):1346-1355, Aug 2011. 